\title{
TWO-PHOTON ABSORPTION \\ IN ANTHRACENE
}

by

\author{
John Peter Hernandez \\ Submitted in Partial Fulfillment \\ of the \\ Requirements for the Degree \\ DOCTOR OF PHILOSOPHY \\ Supervised by Professor Albert Gold \\ Institute of Optics
}

The University of Rochester

Rochester, New York

1966 
The author was born in Madrid, Spain on September 6, 1940. He did his undergraduate work at Manhatten College, receiving a bachelor's degree in electrical engineering in 1962. While at Manhattan College, he held a Juan March Foundation Fellowship. In 1962 the author started graduate study at Stanford University, obtaining a Master of Science in electronic science in 1963. He then transferred to the Institute of Optics at the University of Rochester in 1963 and completed his study for the doctorate in 1966. At Rochester he did research in solid state physics under the direction of Professor Albert Gold. During the academic year 19621963 he held a Stanford Graduate Fellowship. In 1963-1964 he held a teaching assistantship and served as a research assistant from 1964 to 1966. 


\section{ACKNOWLEDGMENTS}

The author would like to express his gratitude to Professor Albert Gold for encouraging him to investigate this problem and for his guidance during the research period. Thanks are due to Professor M. Parker Givens for helpful information on the crystal optics section of this work, and to Dr. R. Pariser who provided the author with his unpublished anthracene wave functions.

It is a pleasure to acknowledge discussions with Dr. H. B. Bebb on his related work and with Mr. T. P. Martin who served as a sounding board in the latter part of this work. Special thanks are due to Dr. T. H. Keil whose physical intuition contributed much to the author's understanding of problems in solid state. 


\begin{abstract}
Two-photon absorption in anthracene has received considerable attention recently. Experimental work on both absorption by the molecule and the crystal has been done and order of magnitude calculations on the absolute intensity of the absorption have been reported. The nature of the final states in the absorption process has been the subject of much debate.

This work presents a calculation of the transition rates based on the best available wave functions. Special attention is paid to the identification of the absorbing states via the polarization dependence of the absorption. The calculation treats both the molecule and the crystal.

Since the calculated eigenstates of the molecule do not correspond in energy to the observed transitions, the theory is extended to include intra-molecular vibrations. The position, splittings and wave vector dependence of the exciton levels are calculated, for the first time, for even parity electronic states. The anisotropy of the crystal is carefully taken into account. The spectroscopic identification of the final states in two-photon absorption in anthracene is the primary purpose of this work. The results of proposed experiments are detailed to identify a $\mathrm{B}_{\mathrm{Ig}}$ electronic state at $4.9 \mathrm{eV}$ and an $\mathrm{A}_{\mathrm{Ig}}$ state at $5.0 \mathrm{eV}$ in the molecule. A Davydov doublet derived from the Alg state of the
\end{abstract}


molecule is predicted near $4.8 \mathrm{eV}$ in the crystal and its characteristic absorption is derived.

Based on available experimental information we make the tentative assignments of the observed absorption to a $\mathrm{B}_{\mathrm{g}}$ state at $3.58 \mathrm{eV}$ and an $\mathrm{Ag}$ state at $3.48 \mathrm{eV}$. These exciton states are derived from the $B_{1 g}$ electronjc state of the molecule at $4.94 \mathrm{eV}$. In the molecule, the assignment of the observed transitions is to a vibronic mixture of the lowest energy $B_{2 u}$ state with either or both of the $\mathrm{B}_{1 \mathrm{~g}}$ state at $4.9 \mathrm{eV}$ and the $\mathrm{A} I \mathrm{~g}$ state at $5.0 \mathrm{eV}$. Further experiments are suggested to test these assignments. 
TABLE OF CONTENTS

\section{CHAPTER}

I. INTRODUCTION

II. TWO-PHOTON ABSORPTION

1. Introduction

2. Theory

6

3. Angular Dependence 9

a. B.g Final State 10

b. Alg Final State 12

III. MOLECULAR ABSORPTION

2. Introduction 1 it

2. Eigenstates 15

a. Electronic 15

3. Two-photon Absorption 18

a. Bg Final State 19

b. Alg Final State 23

4. Conclusion $2 ?$

IV. EXCTTONS

1. Introduction 32

2. Higenstates $3 ?$

a. Wave functions $3 ?$

b. Energies 33

3. Crystal Optics 56

4. Two-photon Atsorption 61 
CHAPTER

Page

5. Conclusion

V. VIBRONIC STATES

1. Introduction

2. Eigenstates

3. Two-photon Absorption

VI. CONCLUSION

APPENDICES

A. ELECTRONIC WAVE FUNCTIONS

84

B. RANDOM SAMPLE AVERAGES 90

C. MATRIX EIENENTS

34

D. POSITION OF MOLECUEES

96

E. WAVE VECTOR DEPENDENCE OF ENERGY

99

REFERENCES 


\section{LIST OF TABLES}

Table No.

Page

2-1 Character Table for $D_{2 h}$ (Ref. 32)

3-1 Ratios of Absorption: $\frac{W \text { circular }}{W \text { linear }}$ and $\frac{W \text { linear }}{W \text { unpolarized }}$ for $A_{1 g}$ Final States

4-1 Anthracene Matrix Elements for Exciton Energies and Transition Probabilities

4-2 Contributions to the Exciton Energy Shifts in First and Second Orders

4-3 Character Table for $\mathrm{C}_{2 h}$

4-4 Symmetry of Exciton States

4-5 Parameters for Two-Photon Transitions to $\mathrm{A}_{\text {Ig-Derived Exciton States }}$

5-1 Selected Normal Mode Vibrational Frequencies of the Anthracene Molecule and Temperature Dependence

A-1 Coefficients for the Construction of Anthracene Molecular Orbitals

A-2 Configuration Interaction Coefficients for the Construction of the Even Parity States of the Anthracene Molecule

D-1 Positions of the Molecules in the Anthracene Crystal to a Distance of $50 a_{0}$

E-1 Wave Vector Dependence of the Exciton States Derived from the $\mathrm{B} I g(4.9 \mathrm{eV})$ state of the Molecule, First and Second Order

E-2 Wave Vector Dependence of the Exciton States Derived from the Alg $(5.0 \mathrm{eV})$ State of the Molecule, First and Second Order 
3-1 Carbon skeleton of the aromatic hydrocarbons

3-2 Variation of two-photon absorption with ellipticity of exciting light. $B_{1 g}$ final state $\mathrm{Cf}$. equations $3-12,3-14$ and $3-17$

3-3 Variation of two-photon absorption with ellipticity of exciting light. Alg $(5.0 \mathrm{eV})$

f'inal state $\mathrm{Cf}$. equations $3-18 a, 3-22$ and $3-24$

3-4 Variation of two-photor atsorption with ellipticity of exciting light. Alg $(6.8 \mathrm{ev})$ final state $\mathrm{Cf}$. equations $3-18 \mathrm{a}, 3-22$ and $3-24$

4-1 Unit cell of anthracene

4-2 Orientation of the molecules within the unit cell of anthracene. See reference 39

4-3 Convergence of the lattice sum $D$ for the $4.9 \mathrm{eV}$ state

Convergence of the lattice sum $D$ for the $5.0 \mathrm{eV}$ state

4-5 Convergence of the lattice sum $D$ for the $6.8 \mathrm{eV}$ state

4-6 Convergence of the lattice sums $\Delta$ and d at $\mathrm{k}=0.4 .9 \mathrm{eV}$ state

4-7 Convergence of the lattice sums $\Delta$ and d at $\mathrm{k}=0 . \quad 5.0 \mathrm{eV}$ state

Convergence of the Inttice sums $\Delta$ and $\dot{a}$ at, $k=0.6 .8 \mathrm{eV}$ state

4-9 Energy shifts and splittings, quadrupolequadrupole first order calcuiation. B $>u$ and 33 levels are experimentally determined. Cf. references 49 and 53

4-10 Energy shifts and splittings, guadrupolequadrupole plus maximum Van der Waals calculation. $B_{21}$ and $B_{3} u$ levels are experimentally determined. Cf. references 49 and 53 
4-I1 Energy shifts and splittings, quadrupolequadrupole plus "reasonable" Van der Walls contribution. $\mathrm{B}_{2} \mathrm{u}$ and $\mathrm{B}_{3} \mathrm{u}$ levels are experimentally determined. Cf. references 49 and 53

4-12 Definition of the angle $\alpha$

4-13 Important directions in crystalline anthracene

5-1 Vibronic states of molecular anthracene and comments on two-photon absorption

A-l Carbon skeleton of anthracene, molecular parameters 
CHAPTER I

\section{INTRODUCTION}

The theory of two-photon absorption by an atomic system was originally formulated by Goeppert-Mayer ${ }^{1}$ in 1931. Observation of the process followed by thirty years with the experiment of Kaiser and Garrett ${ }^{2}$ in $\mathrm{Ca} \mathrm{F}_{2}: \mathrm{Eu}^{++}$. Since that first experiment, using the extremely high light intensities available from lasers, interest in multi-photon processes was renewed and observations have far outpaced calculations.

Some of the observations of two-photon absorption are those of Abella ${ }^{3}$ in Cs, Peticolas et $\mathrm{al}^{4}$ in $\mathrm{C}_{14} \mathrm{H}_{10}$, Giordmain and Howe in $\mathrm{C} \mathrm{S}_{2}$, Braunstein and Ockman ${ }^{6}$ in $\mathrm{Cd} S$ and several liquids, and Hopfield et $a I^{7}$ in KI and Cs I. Theoretical work has been done by Kleinman ${ }^{8}$ on atomic systems; Braunstein, ${ }^{9}$ Loudon, ${ }^{10}$ and Hopfield and Worlock $^{7}$ on solids. Finally, theory and calculations on specific systems have been done by Axe $\mathrm{Al}^{11}$ on complex atoms, Bebb and Gold ${ }^{12}$ on rare gases and hydrogen for higher order processes, Zernik ${ }^{58}$ on hydrogen, Evleth and Peticolas ${ }^{13}$ on molecules, and Gold and Hernandez 14 on atoms in solids. Other multi-photon processes which have received considerable attention are harmonic generation and other non-linear processes, the work has been recently reviewed by Franken and Ward. ${ }^{15}$ This thesis presents a calculation of the two-photon absorption of anthracene. The work separates into a computation of two-photon 
absorption by the molecular system and the extension to the crystal. The shifts and splittings of the electronic states in going from the gas to the crystalline phase are calculated. The polarization and angular dependence of the absorption is detailed.

Since the even parity electronic states of the anthracene molecule are predicted to be at considerably higher energies than those at which transitions are observed, we suggest that absorption by the molecule is due to perturbations of the electronic states by vibrations. The characteristics of these vibronic states are studied here. On the other hand, the exciton shifts calculated for the solid suggest that the observations on solid samples are of two-photon absorption directly to even parity states. Throughout the work we shall concentrate on the symmetry characteristics of the two-photon absorption.

The observation of two-photon absorption by anthracene was first made by Peticolas, Goldsborough and Rieckhoff, ${ }^{4}$ further experiments were carried out by Peticolas and Rieckhoff ${ }^{16}$ and by Peticolas, Norris and Rieckhof $f^{17}$ in dilute solutions of anthracene in EPA glass; by Singh and Stoicheff, ${ }^{18}$ Hall, Jennings and McClintock ${ }^{19}$ and Singh, Jones, Siebrand, Stoicheff and Schneider ${ }^{20}$ in crystals, and by Iannuzzi and Polacco 21,22 in powdered samples. All these experiments measured the blue fluorescence following two-photon absorption. Most recently, Frohlich and Mahr observed the process directly in absorption, with a crystalline sample. Order of magnitude calculations of the absorption have been reported by the experimenters and a review of the work, up to 1965 , has appeared. 20 
There has been controversy from the start as to the nature of the final state in the absorption process. Two processes have been discovered which result in blue fluorescence after irradiation with red, ruby laser, light. The first, of interest to us, is twophoton absorption with a fluorescent lifetime of 26 nanoseconds. 19 The second is bi-molecular triplet annihilation with a fluorescent lifetime of 17.2 milliseconds. ${ }^{19}$ After these two processes had been separated, the controversy over the nature ${ }^{24}$ and symmetries $21,25,22,24$ of the final state in the two-photon absorption process continued and has not been resolved unambiguously. It is part of the purpose of this work to propose more definitive experiments.

Our general plan is as follows. Chapter II contains a general, brief, description of the semi-classical theory of twophoton absorption. The polarization dependence is isolated and $D_{2 h}$, the group of anthracene, is used as an example to show how group theory may be invoked to predict the angular dependence of transitions between states of specific symmetries. The chapter concludes by giving expressions which are applied to anthracene in the succeeding chapters.

Chapter III describes the electronic eigenstates of alternate hydrocarbons in the context of the Pariser-Parr ${ }^{26}$ semi-empirical, $\pi$-electron, molecular orbital theory including configuration interaction. The results of Pariser ${ }^{27}$ for the lowest, even parity, states of anthracene are given. These provide us with the wave functions to be used in this work. Finally, the selection rules for two-photon absorption in molecular anthracene are developed and the polarization 
dependence of the different possible transitions detailed. Averages are performed to predict the absorption characteristics of samples consisting of randomly oriented, non-interacting, molecules as an approximation of gas or liquid samples.

Chapter IV deals with crystalline anthracene. Wave functions for the exciton states are constructed in the Frenkel context. The energy shifts of the states on passing to the solid phase are calculated in lowest order and higher order contributions are estimated. It is found that, in contrast to the molecule, the even parity states may lie in the energy region which has been investigated experimentally. We discuss, briefly, crystal optics in anthracene as a preliminary step in the discussion of the absorption spectrum of the crystal which follows. The angular dependence of the absorption is predicted and found consistent with recent experimental results. Chapter $\mathrm{V}$ investigates the consequences of allowing molecular vibrations to $\mathrm{mix}$ the electronic states of the molecule. It is suggested that the observed transitions take place to vibronic states consisting of an admixture of even parity states into lower energy odd parity states. Symmetry characteristics and the possible identification of the vibrations causing the mixing are investigated in detail.

In summary, we conclude that the final state in two-photon absorption by the anthracene molecule is an admixture, due to intramolecular vibrations, of the lowest energy $\left(B_{2 u}\right)$ electronic state with either or both of the $B_{1 g}$ electronic state at $4.9 \mathrm{eV}$ and the $\mathrm{Alg}$ electronic state at $5.0 \mathrm{eV}$ predicted by Pariser. 27 In contrast, 
we identify the states observed by Frohlich and Mahr ${ }^{55}$ in crystalline anthracene with an $\mathrm{A}_{\mathrm{g}}$ state at $3.48 \mathrm{eV}$ and a $\mathrm{B}_{\mathrm{g}}$ state at $3.58 \mathrm{eV}$. These exciton states are derived from the $B_{1 g}$ electronic state of the molecule at $4.9 \mathrm{eV}$. 
CHAPTER II

TWO-PHOTON ABSORPTION

\section{Introduction}

In this chapter the theory of two-photon absorption is reviewed. The formulation is semi-classical, the emphasis will be on symmetry and the polarization dependence of the two-photon process. The expressions obtained in this chapter will be applied in the succeeding chapters to absorption by molecular and crystalline anthracene. We specialize the theory to absorption of two photons of the same energy but quite general polarization. The technique of using an average energy for the intermediate states in the two-photon absorption process is used to extract the polarization dependence. ${ }^{14}$ $\mathrm{D}_{2 h}$, the group of the anthracene molecule, is used as an example. An identification of the final state in an absorption process is sought via the polarization dependence.

\section{Theory}

The Hamiltonian of an electron in a molecular system and its interaction with the electro-magnetic field is well known to be

$$
H=\frac{\left(p-\frac{e}{c} \underline{A}(\underline{r})\right)^{2}}{2 m}+V(\underset{\sim}{r})
$$

where $e, m$, and $c$ have their usual meaning; $\underline{p}$ is the momentum 
operator, $\underline{r}$ the position operator, $\underline{A}(\underline{r})$ the vector potential of the field, and $V(\underline{r})$ the self-consistent potential of the molecular system. The expression in $(2-1)$ can be expanded to give

$$
H=H_{0}+H^{\prime}=\left(\frac{p^{2}}{2 m}+V(\underset{\sim}{r})\right)-\frac{e}{m c} \underset{\sim}{A}(r) \cdot p+\frac{e^{2}}{2 m c^{2}}{\underset{\sim}{A}(\underset{\sim}{r})}^{2}
$$

consistently using the Coulomb gauge

$$
\varphi=0, \quad \nabla \cdot A_{\sim}(\underline{r})=0
$$

This Hamiltonian may, completely equivalently, be replaced by

$$
H=H_{0}-e \xi(r) \cdot r
$$

where $\xi(\underline{r})$ is the transverse part of the electric field vector. Equations (2-2) and (2-4) are related by a similarity transformation and are equivalent. This equivalence is discussed quite generally by Power and Zienau, 28 and in the dipole approximation by GoeppertMayer, ${ }^{1}$ Richards, 29 and Dirac. 30

The electric field can be expanded in plane waves:

$$
\left.\xi_{\nu}\left(\gamma_{\sim}\right)=\sum\left[\xi_{\lambda}^{0} \hat{\epsilon}_{\lambda} \exp i{\underset{\sim}{k}}_{\lambda} \cdot \underset{\sim}{r}-\omega_{\lambda} t\right)+c . c .\right](2-5)
$$

where $\hat{\epsilon}_{\lambda}$ is the polarization vector, $\underline{k}_{\lambda}$ and $\omega_{\lambda}$ the wave vector and frequency of the $\lambda$ th. component of the field. In the usual way, we shall perturb the system

$$
H_{0}|m\rangle=E_{m}|m\rangle
$$

to obtain the transition rate between states of the unperturbed 
system. The transition rate for the absorption of two photons, one of frequency $\omega_{1}$ and the other of frequency $\omega_{2}$ is obtained in a completely straight forward manner from second order perturbation theory. The result is given by ${ }^{1}$

$$
\begin{aligned}
\omega_{f i} & =2 \pi\left(\frac{e^{2}}{\hbar}\right)^{2}\left|\xi_{1}^{0} \xi_{2}^{0}\right|^{2} \mid \sum_{m} \frac{\left\langle f\left|\hat{\epsilon}_{2} \cdot \underset{\sim}{r}\right| m\right\rangle\left\langle m\left|\hat{\epsilon}_{1} \cdot \underline{\sim}\right| i\right\rangle}{E_{m i}-\hbar \omega_{1}} \\
& +\left.\frac{\left\langle f\left|\hat{\epsilon}_{1} \cdot \underset{\sim}{r}\right| m\right\rangle\left\langle m\left|\hat{\epsilon}_{2} \cdot \underset{\sim}{r}\right| i\right\rangle}{E_{m i}-\hbar \omega_{2}}\right|^{2} \delta\left(\omega_{f i}-\omega_{1}-\omega_{2}\right)_{(2-7)}
\end{aligned}
$$

in the dipole approximation $\left(e^{i \underline{k} \cdot r}=1\right)$. We shall further specialize, for purposes of this work, to photons of the same energy; assume a normalized line shape function for the final state $g(E)$ such that $\int g(E) d E=1$ and introduce the photon flux $F$, from the expression

$$
\left|\varepsilon^{0}\right|^{2}=\frac{2 \pi \hbar \omega}{c} F
$$

Then, from $(2-7)$, the transition rate is given by

$$
w_{f i}=(2 \pi)^{3}\left(\frac{e^{2}}{\hbar c}\right)^{2} \hbar(\hbar \omega)^{2} F_{1} F_{2}\left|R_{f i}^{(2)}\right|^{2} g(2 \hbar \omega)
$$

where

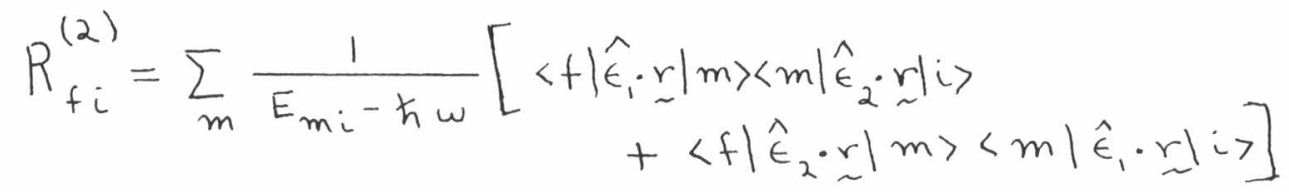

When the transitions take place in a dielectric medium, corrections to $(2-7)$ and $(2-8)$ are required to introduce the local value of the electric field and to correct the velocity of propagation of 
the photons by the index of refraction. 31 Then, (2-9) becomes

$\omega_{f i}=(2 \pi)^{3}\left(\frac{e^{2}}{\hbar c}\right)^{2} \hbar(\hbar \omega)^{2} F_{1} F_{2}\left(\frac{n_{\omega}^{2}\left(\hat{\epsilon}_{1}\right)+2}{3}\right)^{2}\left(\frac{n_{\omega}^{2}\left(\hat{\epsilon}_{2}\right)+2}{3}\right)^{2} \frac{\left|R_{f i}^{(2)}\right|^{2} g(2 E)}{n(\underline{\hat{\epsilon}}) n_{\omega}\left(\hat{\epsilon}_{2}\right)}(2-11)$

where $n_{\omega}(\hat{\epsilon})$ is the index at frequency $\omega$ in the direction of the field.

\section{Angular Dependence}

The angular dependence of the process is contained in the index corrections and in the square of the second order matrix element, these are the terms which will concern us primarily. Let us now concentrate on the second order matrix element. Given the symmetry of the system and of the initial state, we can predict, from the second order matrix element, the possible final states and for each of those the intermediate states with non-vanishing contributions. Thus, given the symmetry of the initial and final states, the angular dependence of the absorption may be predicted. 33 For example, in $\mathrm{D}_{2 \mathrm{~h}}$ (the symmetry of $\mathrm{C}_{14} \mathrm{H}_{10}$ ), the ground state transforms as $\mathrm{A}_{1 \mathrm{~g}}$ (see Table 2-1). Thus the possible final state for two-photon absorption via dipole transitions are: $\mathrm{A}_{1 g}, \mathrm{~B}_{1 g}, \mathrm{~B}_{2 g}$ and $\mathrm{B}_{3 g}$.

In order to isolate the angular dependence, we define

$$
\hat{\epsilon}_{i}=l_{i} \hat{x}+m_{i} \hat{y}+n_{i} \hat{z}
$$

where $x, y$ and $z$ are the basis set of the molecule and $\ell, m$ and $n$ the direction cosines. In anthracene, $x, y$ and $z$ are, respectively, the long, short and normal axes of the molecule. Basis functions 
for the irreducible representations of $D_{2 h}$ are specified in table $2-1$

We shall now investigate $A_{1 g}$ and $B_{1 g}$ final states $\left(B_{2 g}\right.$ and $\mathrm{B}_{3 g}$ are related to $\mathrm{B}_{1 \mathrm{~g}}$ by a simple coordinate rotation).

a. Blg final state

The second order matrix element is given by

$R_{B_{1 g} A_{1 g}}^{(2)}=\sum_{m} \frac{\left\langle B_{1 g}\left|\hat{\epsilon}_{1} \cdot \underline{\sim}\right| m\right\rangle\left\langle m\left|\hat{\epsilon}_{2} \cdot r\right| A_{1 g}\right\rangle+\left\langle B_{1 g}\left|\hat{\epsilon}_{2}-r\right| m\right\rangle\left\langle m\left|\hat{\epsilon}_{1} \cdot r\right| A_{1 g}\right\rangle}{E_{m, A_{1 g}}-\hbar \omega}$.

It is clear from the character table that only the combinations $l_{1}$ $m_{2}$ and $l_{2} m_{1}$ have non-vanishing contributions, thus out of the complete set $m$ only $\mathrm{B}_{2} \mathrm{u}$ and $\mathrm{B}_{3} \mathrm{u}$ intermediate states contribute. Equation $(2-10 a)$ can then be written:

$$
R_{B_{1} A_{1 g}}^{(2)}=\left(l_{1} m_{2}+m_{1} l_{2}\right) R^{(2)}(x y, y x)
$$

where

$$
\begin{aligned}
R^{(2)}(x y, y x) \equiv & \sum_{B_{2 u}} \frac{\left\langle B_{1 g}|x| B_{2 u}\right\rangle\left\langle B_{2 u}|y| A_{1 y}\right\rangle}{\left.E_{32 u, A_{1 g}}\right\rangle \omega} \\
& +\sum_{B_{3 u}} \frac{\left\langle B_{1 g}|y| B_{3 u}\right\rangle\left\langle B_{3 u}|x| A_{1 g}\right\rangle}{E_{3}, A_{1 j}-\hbar \omega} .
\end{aligned}
$$

The transition probability depends on the polarization only

through the function of the direction cosines in equation (2-10b).

Thus, if two experiments are undertaken, the first with polarization vectors $\hat{\epsilon}_{1}^{a}$ and $\hat{\epsilon}$ ? , and the second with $\hat{\epsilon}_{1}^{b}$ and $\hat{\epsilon}$ ?, the ratio of the transition rates is given by 
TABLE 2-1

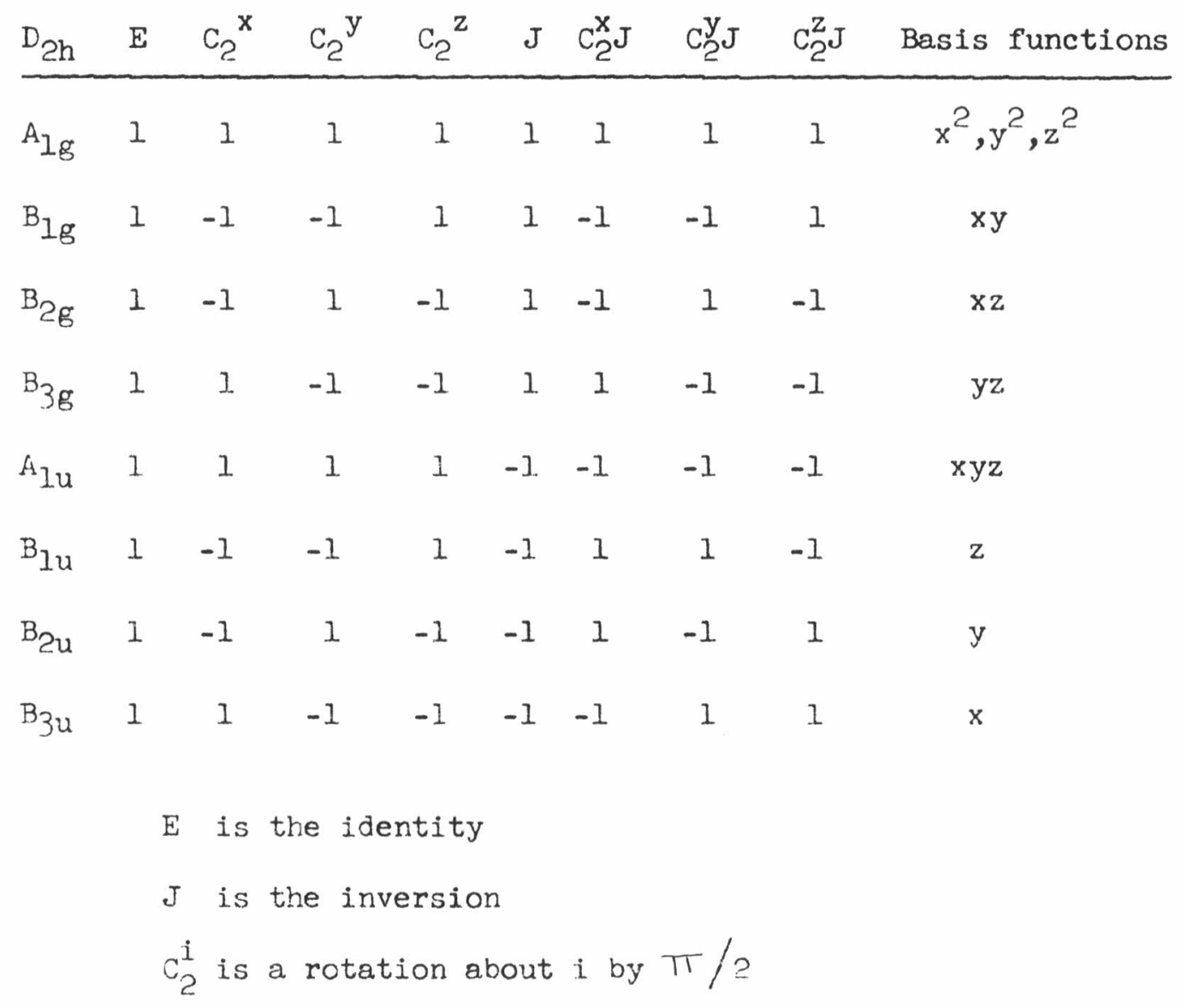




$$
\frac{w^{(a)}}{w^{(b)}}=\frac{\left|l_{1}^{a} m_{2}^{a}+m_{1}^{a} l_{2}^{a}\right|^{2}}{\left|l_{1}^{b} m_{2}^{b}+m_{1}^{b} l_{2}^{b}\right|^{2}}
$$

This ratio is completely determined by symetry therefore permitting unambiguous identification of the $\mathrm{A}_{\mathrm{lg}}$ to $\mathrm{B}_{\mathrm{lg}}$ transition.

\section{b. Ag final state}

The second order matrix element is given by

$$
R_{A_{i g}^{*} A_{1 g}}^{(2)}=\sum \frac{\left\langle A_{i g}^{*}\left|\hat{\epsilon}_{1} \cdot r\right| m\right\rangle\left\langle m\left|\hat{\epsilon}_{2} \cdot r\right| A_{1 g}\right\rangle+\left\langle A_{1 g}^{*}\left|\hat{\epsilon}_{2} \cdot r\right| m\right\rangle\left\langle m\left|\hat{\epsilon}_{1} \cdot \underline{r}\right| A_{1 g}\right\rangle}{E_{m, A_{1 g}}-\hbar \omega}(2-10 c)
$$

using the character table once again

$$
\begin{gathered}
R_{A_{i g}^{*} A_{1 g}}^{(2)}=l_{1} l_{2} \sum_{B_{3 u}} \frac{\left\langle A_{1 g}^{*}|x| B_{3 u}\right\rangle\left\langle B_{3 u}|x| A_{1 g}\right\rangle}{E_{B_{3 u}, A_{1 g}}-\hbar \omega}+m_{1} m_{2} \sum_{B_{2 u}} \frac{\left\langle A_{1 g}^{*}|y| B_{2 u}\right\rangle\left\langle B_{2 u}|y| A_{1 g}\right\rangle}{E_{2 u} A_{1 g}^{-\hbar \omega}} \\
+n_{1} n_{2} \sum_{B_{1 u}} \frac{\left\langle A_{1 g}^{*}|z| B_{1 u}\right\rangle\left\langle B_{1 u}|z| A_{1 g}\right\rangle}{E_{B_{1 u}, A_{1 g}}} \\
\end{gathered}
$$

It can be seen from $(2-10 d)$ that the angular dependence is not determined by symmetry alone and that the matrix elements, or at least ratios of the matrix elements, must be calculated. Continuing, with obvious definitions,

$$
R_{A_{\text {Ig }}^{*} A_{1 g}}^{(2)}=l_{1} l_{2} R^{(2)}(x x)+m_{1} m_{2} R^{(2)}(y y)+n_{1} n_{2} R^{(2)}(z z)
$$


We shall evaluate $R^{(2)}(x x)$, for example, by setting

$$
R^{(2)}(x x)=\frac{\left\langle A_{i g}^{*}\left|x^{2}\right| A_{1 g}\right\rangle}{E_{x x}-\hbar \omega}
$$

where $\bar{E}(x x)$ is the mean energy (defined by 10d, 14, and 15) of the $B_{3 u}$ states of the system. The mean energy approach is well known in the theory of dispersion forces ${ }^{34}$ and is essentially a variational solution of the time dependent Schrodinger equation. 35 A similar technique has been employed by Axe ${ }^{11}$ and by Bebb and Gold. ${ }^{12}$ The approximation in our method is that we shall choose, rather than calculate the average energies. The polarization dependence for $\mathrm{A}_{\text {Ig }}$ to $A_{1 g}^{*}$ transitions is in the square of equation $(2-14)$. 
CHAPTER III

MOLECULAR ABSORPTION

\section{Introduction}

We discuss the construction of the eigenstates of polyacene molecules here and then use these states to calculate the two-photon absorption of a random, molecular sample. The first section reviews the semi-empirical, $\pi$-electron, molecular orbital theory, including configuration interaction, due to Pariser and Parr 26,27 for the construction of electronic states of alternate hydrocarbons. Pariser 27 has applied the theory to anthracene and his wave functions are discussed.

The concluding section of the chapter uses the states previously described to calculate two-photon transition rates. The entire discussion is then applied to absorption by molecular anthracene, including predictions on the angular dependence and symmetry characteristics of the absorption.

As far as two-photon absorption is concerned, the most interesting part of this chapter is the results which permit the identification of the final state in the process. In summary, $B_{1 g}$ final states can be unambiguously identified by the relative strengths of the absorption induced by light beams of different polarization character. For example, the ratio of the absorption induced by 
circularly polarized light to that induced by linearly polarized light is $3 / 2$ for all $\mathrm{B}_{\text {Ig }}$ final states; this is a consequence of symmetry alone. When two-photon absorption at some energy has characteristics which differ from those predicted for $\mathrm{B}_{1 \mathrm{~g}}$ final states the transitions must be due to an $A_{1 g}$ state. Specific application is made to the three lowest lying even parity electronic states of anthracene predicted by Pariser.

Since the absorption observed, to date, is at an energy that does not correspond to any of the states predicted by Pariser, the results derived in this chapter are of interest for future experiments. To deal with the experiments already performed, we shall extend these results by introducing the effects of molecular vibrations in Chapter $V$.

\section{Eigenstates}

\section{a. Electronic}

Consider molecules which are planar and made up of carbon and hydrogen atoms. For example, benzene $\left(\mathrm{C}_{6} \mathrm{H}_{5}\right)$, napthalene $\left(\mathrm{C}_{10} \mathrm{H}_{8}\right)$, anthracene $\left(\mathrm{C}_{14} \mathrm{H}_{10}\right)$, napththacene $\left(\mathrm{C}_{18} \mathrm{H}_{12}\right)$ and penthacene $\left(\mathrm{C}_{22} \mathrm{H}_{1} i_{4}\right)$. The carbon skeletons of these molecules are made up of, approximately, regular hexagons (see Figure 3-1). The carbon atoms have the configuration $1 s^{2} 2 s^{2} 2 p^{2}$ and a simplified view of these molecules consists in the assumptions that hybridized orbitals constructed from the $2 s^{2} 2 p \sigma$ wave functions are mainly responsible for the binding of each carbon to its neighbors and the hydrogen. The $2 p \sigma$ electron is the one which lies mostly in the plane of the molecule, contrasted with the 


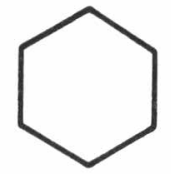

Benzene

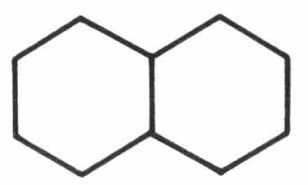

Naphthalene

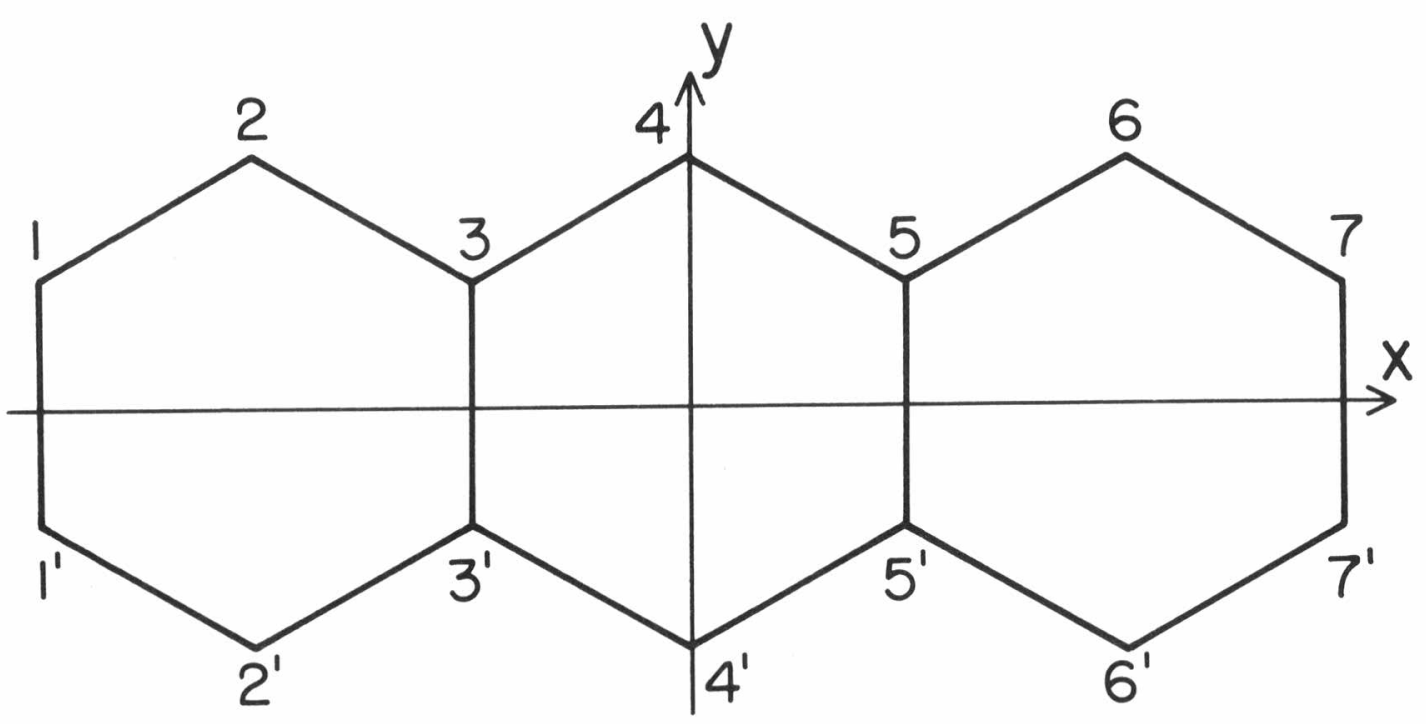

Anthracene<smiles>C1CCC2CC3CC4CCCCC4CC3CC2C1</smiles>

Naphthacene<smiles>C1CCC2CC3CC4CC5CCCCC5CC4CC3CC2C1</smiles>

Pentacene

Fig. 3-I 
$2 \mathrm{p} \pi$ electron which has a node in that plane. These $\pi$-electrons do not overlap strongly with the $\sigma$-electrons or with each other so they are more loosely bound to the molecule and are mainly responsible for the low energy, electronic, properties of the aromatic molecules. The so-called $\pi$-electron approximation consists in building wave functions from linear combinations of atomic orbitals (LCAO) considering only the $\pi$-electrons explicitly. ${ }^{36}$ This method reproduces the gross features of the spectrum but, in general, agreement with experiment is poor. The disagreement is mainly a consequence of a lack of self-consistency in the molecular orbitals.

To reduce the complexity of calculations and to obtain better agreement with experiments, Pariser and $\operatorname{Parr}^{26,27}$ made the theory semi-empirical as described later. Secondly, they neglected differential overlap, which reduces the computational complexity of the problem greatly. Finally, they included the possibility of configuration interaction to compensate for the fact that the molecular orbitals might lack self-consistency. The method works quite well giving correct energies and symmetry identifications, fair agreement on oscillator strengths and correctly predicting polarization ratios which seem to hinge on the inclusion of configuration interaction. 27

Let the carbon atoms be numbered as in Figure $3-1$ and $X_{\mu}$ be the $2 \mathrm{p} \pi$ carbon atomic orbital (AO) centered at atom $\mu$. Then, the molecular orbitals (MO) are constructed:

$$
\phi_{\lambda^{ \pm}}=\sum_{\mu} C_{\lambda^{ \pm} \mu}\left(X_{\mu} \pm X_{\mu^{\prime}}\right) \equiv \sum_{\mu} C_{\lambda^{ \pm} \mu} \sigma_{\mu}^{ \pm}
$$


The coefficients $C_{\lambda \mu}$ are solutions of the Schroedinger equation for

$$
H=\sum_{\mu}\left(H_{\text {core }}\right) \mu+\sum_{p>q} \frac{e^{2}}{r_{p q}}
$$

where $\mathrm{p}$ and $\mathrm{q}$ run over the nearest neighbor $\pi$-electrons only. The solutions for the energy are 37

$$
E_{\lambda}=E_{0}+k_{\lambda} \beta
$$

where $E_{O}$ is the energy of a $2 p \pi$-electron on one carbon, $k_{\lambda}$ is a numerical constant determined from the secular equation and $\beta$ is the nearest neighbor resonance integral. Then, symmetry and orthonormality are invoked to find the $\mathrm{C}_{\lambda \mu} \mu$ 's as a function of $\mathrm{k}_{\lambda} \cdot{ }^{38} \beta$ is one of the three parameters to be fitted for the semi-empirical theory. The analytical formulas for $\mathrm{k}_{\lambda}$ and $\mathrm{C}_{\lambda \mu}$ are given in Appendix A for reference. They are also explicitly tabulated for anthracene. The ground state configuration is constructed by doubly occupying the lowest energy MCs, the singly excited configurations, by raising one electron to a higher energy MO. All the configurations are antisymmetrized. The configurations are allowed to interact via the electrostatic interaction and the one center and nearest neighbor coulomb integrals are fitted. The three parameters are consistently fitted to the lowest three energy levels of benzene and then kept the same for all other molecules. Specializing to anthracene and the wave functions used in this work, it was found that the specific functions used for the 
carbon $2 p \pi$ electron were not important in our computations since neglecting differential overlap reduced matrix elements to dimensions of the molecule, (Pariser used Slater functions in his calculation). However, polarization ratios were found to be quite sensitive to configuration interaction, a fact not surprising since the usual, one-photon, polarization ratios also exhibit this sensitivity.

The relevant wave functions for this calculation are given

in Table A-2 and the molecular parameters ${ }^{39}$ are shown in Figure A-I. An energy level diagram for the molecule according to Pariser is given in Figure 4-9, for example. The odd parity states shown in the figure are experimental, Pariser predicts the $B_{2 u}$ state at $3.64 \mathrm{eV}$ and the $\mathrm{B}_{3 \mathrm{u}}$ state at $5.50 \mathrm{eV}$. Thus, Pariser's predictions for the odd parity states are about $0.2 \mathrm{eV}$ high.

\section{Two-Photon Absorption}

We now use the wave functions of the previous section and the development of Chapter II to calculate the two-photon absorption spectrum of random samples (liquid or gas), of anthracene.

From equation (2-11), the transition rate is given by

$$
w_{f i}=A\left|R_{f i}^{(2)}\right|^{2} g(2 \hbar \omega)
$$

where,

$$
A=(2 \pi)^{3}\left(\frac{e^{2}}{\hbar c}\right)^{2} \hbar(\hbar \omega)^{2} F_{1} F_{2}\left(\frac{n^{2}+2}{3}\right)^{4} \frac{1}{n^{2}} .
$$

Consider transitions between states of the molecule before introducing the vibronic interaction. 


\section{a. B $_{1 g}$ final state}

Using equation (3-11) along with $(2-13)$ and $(2-11 b)$ we

obtain

$$
W_{B_{\lg A_{1 g}}}=A\left|l_{1} m_{2}+m_{1} l_{2}\right|^{2}\left|R^{(2)}(x y, y x)\right|^{2} g(2 \hbar \omega)
$$

with

$$
\begin{aligned}
R^{(2)}(x y, y x)=\sum_{B_{2 u}} \frac{\left\langle B_{1 g}|x| B_{2 u}\right\rangle\left\langle B_{2 u}|y| A_{1 g}\right\rangle}{E_{B_{2 u}, A_{1 g}-\hbar \omega} \omega} \\
+\sum_{B_{3 u}} \frac{\left\langle B_{1 g}|y| B_{3 u}\right\rangle\left\langle B_{3 u}|x| A_{1 g}\right\rangle}{E_{B_{3 u}}, A_{1 g}-\hbar \omega}
\end{aligned}
$$

We will first obtain the angular dependence of the transition rate for different types of polarization of the incident light. We must average the transition rate over orientations of the molecules in the sample, (see Appendix B for the averages).

i. Unpolarized light

$$
\underset{\epsilon_{1}}{A_{v}} A_{2}\left|l_{1} m_{2}+m_{1} l_{2}\right|^{2}=\frac{1}{9}
$$

ii. Elliptically polarized light

If $\hat{\epsilon}_{1}=\hat{\epsilon}_{2}$ and $\hat{\epsilon} \equiv \frac{\hat{x}+i a \hat{y}}{\sqrt{1+a^{2}}}$, we shall call "a" the ellipticity. $a=0$ is linearly polarized light, $a=1$ is circularly polarized light. Then,

$$
A_{\epsilon}|2 \operatorname{lm}|^{2}=\frac{1}{\left(1+a^{2}\right)^{2}} \frac{a^{4}+4 a^{2}+1}{15}
$$

which for linearly polarized light $(a=0)$ is

$$
\epsilon_{\text {lin }}|2 \operatorname{lm}|^{2}=\frac{4}{15}
$$


and for circular polarized light $(a=1)$,

$$
\operatorname{Av}_{\text {circ }}|2 \operatorname{lm}|^{2}=\frac{2}{5} .
$$

For the variation of the transition rate with ellipticity see Figure 3-2.

To obtain the signature of a $\mathrm{B}_{1 g}$ final state we will look to ratios of the transition rate due to different types of polarization of the incident light, (Cf. Equation (2-14)). For example,

$$
\frac{W_{B_{1 g} A_{1 g}}(\text { ircular })}{W_{B_{1 g}} A_{\text {ig }}(\text { inear })}=\frac{3}{2}
$$

and

$$
\frac{W_{B_{1 g} A_{1 g}(\text { linear })}}{W_{B_{1 g} A_{1 g} \text { (unpolarized) }}}=\frac{4}{5} .
$$

Other ratios may be obtained from Figure 3-2.

Finally, to obtain absolute transition rates we must put numerical values into equation $(3-12)$ while computing $(3-12 a)$. To compute $(3-12 a)$, we shall use

$$
R^{(2)}(x y, y x)=2 \frac{\left\langle B_{1 g}|x y| A_{1 g}\right\rangle}{E_{x y}-\hbar \omega}
$$

where $\bar{E}_{x y}$ is defined by comparing $(3-16)$ and $(3-12 a) . \bar{E}_{x y}$ is a function of $\omega$, but for $\hbar \omega$ far from resonance with any molecular state, we shall approximate the average energy by the first excited state with an allowed transition in the molecule. An exact calculation of the average energy in hydrogen ${ }^{12}$ gives us confidence in 


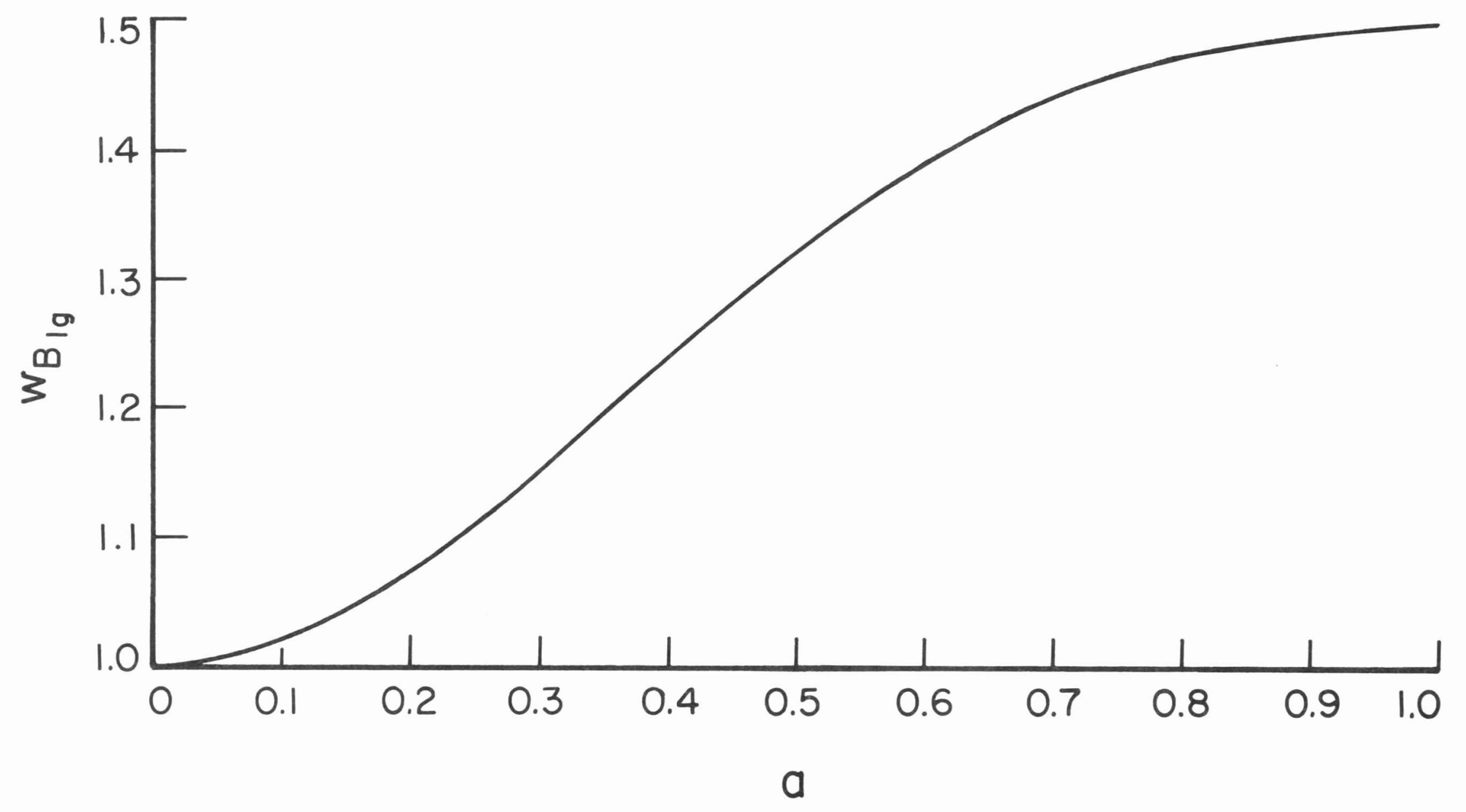

Fig. 3-2

ก 
this approximation. The evaluation of the matrix element will be delayed until the next chapter where all the necessary matrix elements will be presented. There we find

$$
\left\langle B_{19}|x y| A_{19}\right\rangle=7.79 a_{0}^{2}
$$

using Pariser's ${ }^{27}$ wave functions for the $B_{I_{g}}$ state at $4.9 \mathrm{eV}$.

In summary, we shall use; $n=1.8,45 \hbar \omega=1.7 \mathrm{eV}$,

$\bar{E}_{x y}=3.3 \mathrm{ev}^{46}$ and, in the absence of experimental measurement, $g(2 \hbar \omega)=(0.3 \mathrm{eV})^{-1}$

$$
W_{B_{\lg } A_{1 g}}=5.64 \times 10^{-48} F_{1} F_{2} A_{v}\left|l_{1} m_{2}+m_{1} l_{2}\right|^{2} \sec ^{-1}(3-17)
$$

at $2 \hbar \omega=4.9 \mathrm{eV}$ for a random sample, with $F_{1,2}$ in photons $/ \mathrm{cm}^{2}$ sec.

\section{b. Alg final state}

Using equations (3-11) along with $(2-12)$ and $(2-14)$, the transition rate is given by

$$
W_{A_{1 g}^{*} A_{1 g}}=A\left|l_{1} l_{2} R^{(2)}(x x)+m_{1} m_{2} R^{(2)}(y y)+n_{1} n_{2} R^{(2)}(z z)\right|^{2}
$$

In order to compute the angular dependence the matrix elements must be calculated. The $\pi$-electron approximation to the wave functions implies $\mathrm{P}^{(2)}(z z)=0$.

Defining the average energies,

$$
R^{(2)}(x x)=\frac{\left\langle A_{1 g}^{*}\left|x^{2}\right| A_{1 g}\right\rangle}{\bar{E}_{x x}-\hbar \omega}
$$

and

$$
R^{(2)}(y y)=\frac{\left\langle A_{\mid g}^{*}\left|y^{2}\right| A_{1 g}\right\rangle}{\bar{E}_{y y}-\hbar \omega}
$$


Further, for simplicity of notation,

$$
B=\frac{\left\langle A_{1 g}^{*}\left|y^{2}\right| A_{1 g}\right\rangle}{\left\langle A_{i g}^{*}\left|x^{2}\right| A_{1 g}\right\rangle}
$$

which will be calculated, and

$$
\lambda=\frac{\bar{E}_{x x}-\hbar \omega}{\bar{E}_{y y}-\hbar \omega}
$$

which is an unknown parameter. If the average energies are taken as equal (say the first ionization potential) then $\lambda=1$, however, a different and probably more accurate estimate is to take the average energy as being near the energy of the first allowed (in one photon) transition of the correct symmetry. For the later choice in anthracene $\lambda=2.2$. In any case we shall parametrize the angular dependence of filg to $A_{\mathrm{lg}}^{*}$ transitions and show the results for $\lambda=1,1.5,2,2.5$ and 3. It should be observed that despite the parametrization the angular dependence of $\mathrm{A}_{\mathrm{Ig}}$ transitions can be differentiated from that of $\mathrm{B}_{\mathrm{Ig}}$ states described previously (see Table 3-1).

$$
\text { Using equations 3-18 to 3-20b, we obtain for the transition }
$$

rate:

$$
W_{A_{1} A_{1} g}=A\left|R^{(2)}(x x)\right|^{2}\left|l_{1} l_{2}+\lambda B m_{1} m_{2}\right|^{2} g(2 \hbar \omega)
$$

Now we perform averages over orientations of the molecule to obtain:

i. Unpolarized light

$$
\underset{\epsilon_{1}}{A} \epsilon_{2}\left|l_{1} l_{2}+\lambda B m m_{1} m_{2}\right|^{2}=\frac{1}{9}\left[1+(\lambda B)^{2}\right]
$$





\section{ii. Elliptically polarized light}

$$
\begin{array}{r}
A v\left|l_{1} l_{2}+\lambda B m_{1} m_{2}\right|^{2}=\frac{1}{15\left(1+a^{2}\right)^{2}}\left[\left(3 a^{4}+2 a^{2}+3\right)\right. \\
\left.\quad x\left(1+(\lambda B)^{2}\right)+\frac{\lambda B}{8}\left(21 a^{4}-101 a^{2}+16\right)\right]
\end{array}
$$

which for linearly polarized light $(a=0)$ is

$$
\frac{1}{15}\left[3(\lambda B)^{2}+2(\lambda B)+3\right]
$$

and for circularly polarized light $(a=1)$,

$$
\frac{2}{15}\left[(\lambda B)^{2}-(\lambda B)+1\right]
$$

The variation of the transition rate with $a$ and $\lambda$ is shown in Figures $3-3$ and $3-4 . \quad B$ is calculated from Table $t-1$, and its value is

$$
B_{5.0 \mathrm{eV}}=-0.844 ; B_{6.8 \mathrm{eV}}=-0.132
$$

the energies refer to the $A_{1 g}^{*}$ states of Pariser. 27 Table $3-1$ gives ratios of the transition rates for several polarizations.

$$
\text { Before obtaining absolute values of the transition rates to }
$$

${ }^{*}$ lg final states, we wish to emphasize that the results obtained thus far in this section, compared to those of the previous section, permit identification of the symmetry of the final state in twophcton transitions. The identification is best made via the ratios $\frac{w(a=1)}{w(a=0)}$ and $\frac{w(a=0)}{w(\text { unpolarized })}$. If it is established that the final state is $B_{1 g}$ then the 



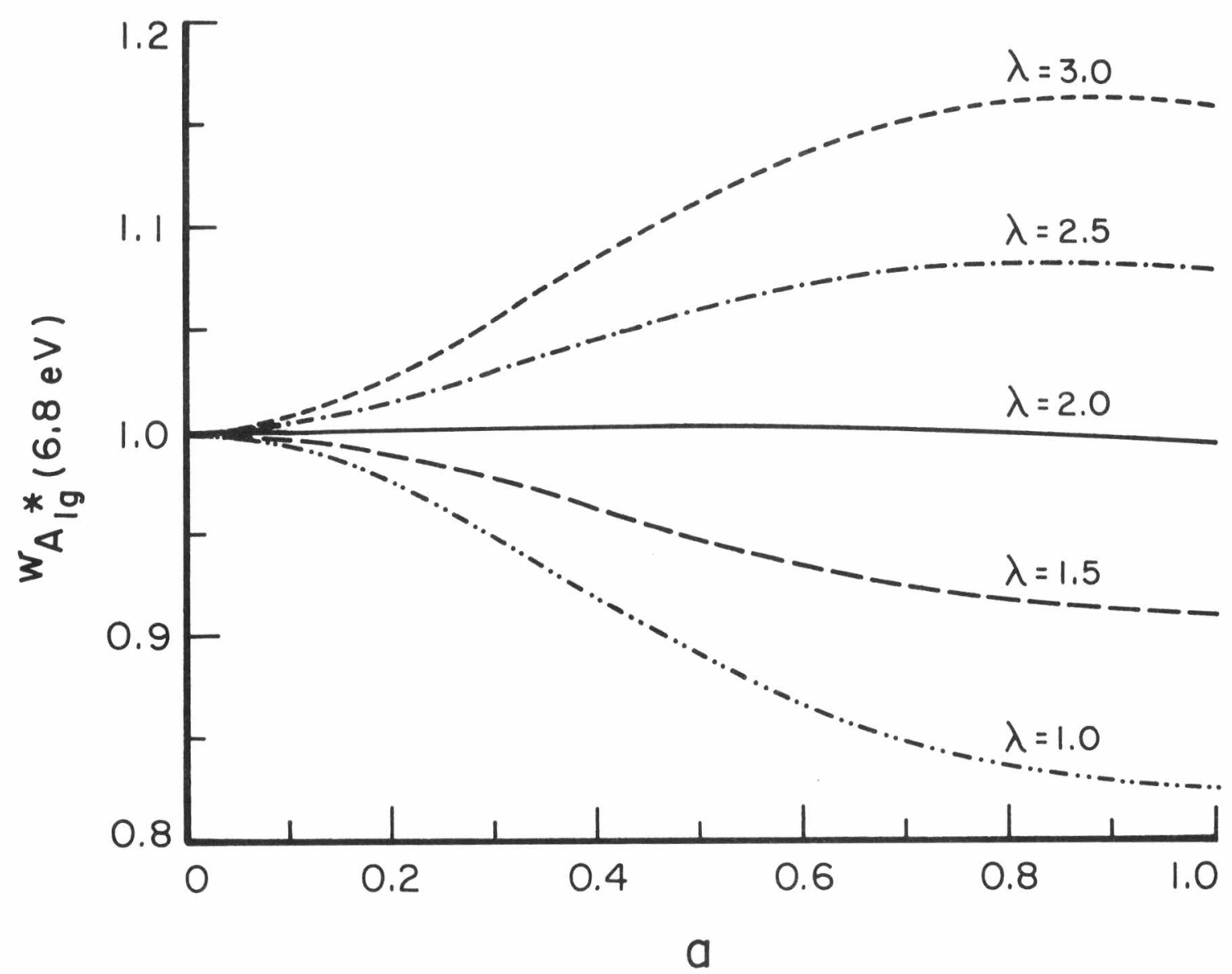

Fig. 3-4 
absolute transition rate will be the test of the average energy and the matrix element calculated. If, however, the final state is not $B_{1 g}$ then it must be $A_{1 g}$ and $\lambda B$ can be fitted via the ratios, while the absolute rate can test the matrix element and the average energy. The absolute transition rate is calculated below.

Using equation $3-18 a$, the averages calculated above, and the same values of $n, \omega$, and $g$ as in the $B_{1 g}$ calculation, along with $\overline{\mathrm{E}}_{\mathrm{XX}}=5.24 \mathrm{eV}$ and

$$
\begin{aligned}
\left\langle A_{19}^{*}\left|x^{2}\right| A_{1 g}\right\rangle= & -1.22 a_{0}^{2} \quad(5.0 \mathrm{eV}) \\
& +16.87 \mathrm{a}_{0}^{2} \quad(6.8 \mathrm{eV})
\end{aligned}
$$

from Table 3-1, we obtain for the transition rates:

$$
\begin{aligned}
W_{A_{1 g}^{*} A_{1 g}} & =2.60 \times 10^{-50} F_{1} F_{2} A_{\epsilon}\left|l_{1} l_{2}+\lambda B_{5} m_{1} m_{2}\right|^{2} \sec ^{-1} .\left(5.00 V V_{3}-24\right) \\
& =4.95 \times 10^{-48} F_{1} F_{2} A_{\epsilon}\left|l_{1} l_{2}+\lambda B_{6.8} m_{1} m_{2}\right|^{2} \sec ^{-1}(6.8 e V)
\end{aligned}
$$

with $\mathrm{F}_{1}$ and $\mathrm{F}_{2}$ in photons $/ \mathrm{cm}^{2}$ sec.

\section{Conclusion}

Note that before these results can be applied to the experiments in random samples, available up to now, they must be modified by the influence of vibrations. These modifications are carried out in Chapter 5 .

In summary, we have caiculated the transition rates to the three lowest energy even parity electronic states of anthracene. The polarization dependence of the absorption, which we predict, should 
give a positive identification of the states. The rates calculated and the energies of the two lowest energy states ( 4.9 and $5.0 \mathrm{eV}$ ) should allow their experimental observation. 
CHAPTER IV

EXCITONS

\section{Introduction}

We first discuss the construction of the eigenstates of a crystal. The approach is the Frenkel exciton 47 formalism specialized to two molecules per unit cell. ${ }^{48}$ Application to $\mathrm{C}_{14} \mathrm{H}_{10}$ is made with emphasis on even parity states. The energies, splittings and dependence on wave vector of the three lowest even parity states of anthracene are calculated and discussed.

A brief review of crystal optics follows since effects arising from the anisotropy of crystalline anthracene are critical to the calculation of its two-photon absorption spectrum. The twophoton spectrum is calculated with emphasis, once again, on symmetry and angular dependence.

The calculation of the energy shifts and splittings suggests the identification of the $\mathrm{B}_{1 g^{-}}$-derived pair of states with those observed by Frohlich and Mahr. 55 They observe a state at $3.58 \mathrm{ev}$ with both light beams polarized along the b direction and another state at $3.48 \mathrm{eV}$ which does not absorb with both beams polarized along $\mathrm{b}$. The identification of the $3.58 \mathrm{eV}$ state with a $B_{\mathrm{g}}$ state and the $3.48 \mathrm{ev}$ state with an $\mathrm{A}_{\mathrm{g}}$ state, both derived from the $\mathrm{B}_{1 \mathrm{~g}}$ molecular Ievel, is shown to be completely consistent with their observations. The observation that the absorption of the solid is quite stronger 
than that of liquid samples ${ }^{56}$ also points to the identification of the states of the crystal with even parity electronic states, in contrast to the vibronic nature of the molecular absorption. Further experiments are suggested and the results predicted.

2. Eigenstates 47,48

\section{a. Wave functions}

Consider the Hamiltonian of an isolated molecule, and its

wave functions

$$
H_{m}(r) \Phi_{i}\left(r_{\sim}\right)=E_{i} \Phi_{i}(r)
$$

We shall take, as a zero order Hamiltonian for the crystal, a sum of those of the individual molecules.

$$
H_{0}=\sum_{(l, \alpha)=1}^{2 N} H_{m}\left(r_{l \alpha}-R_{l_{l \alpha}}\right)
$$

where $l$ runs over the unit cells and $\alpha$ over the inequivalent moiecules within the unit cell. $\mathrm{R}_{\mathrm{l} \alpha}$ is the vector to the $(l \alpha)$ molecule and $\mathrm{Il}_{\alpha}$ the electronic coordinate. The solutions to this zero order crystal problem are functions consisting of products of the molecular function centered at the appropriate sites. Thus for the ground state

$$
\Psi_{0}(r)=\prod_{(l, \alpha)=1}^{2 N} \Phi_{0}\left(r_{\sim} l_{\alpha}-R_{\sim l_{\alpha}}\right)
$$

while for the $(m \beta)$ molecule in the $i^{\text {th }}$ excited state 


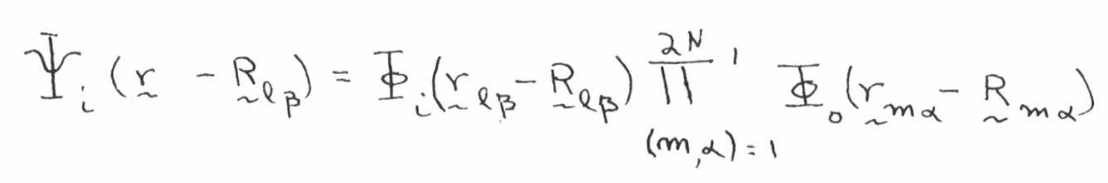

We now take advantage of the translational invariance to construct the excited states out of the $2 \mathrm{~N}$-fold degenerate set in (4-4)

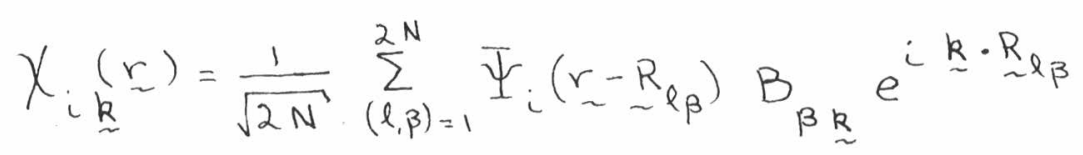

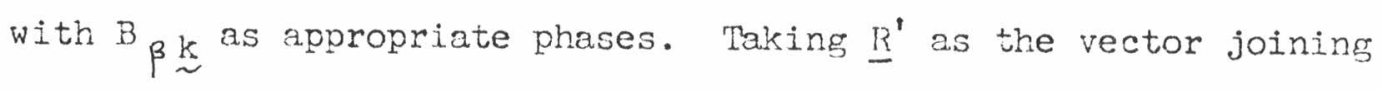
the two molecules in a unit cell we obtain two solutions

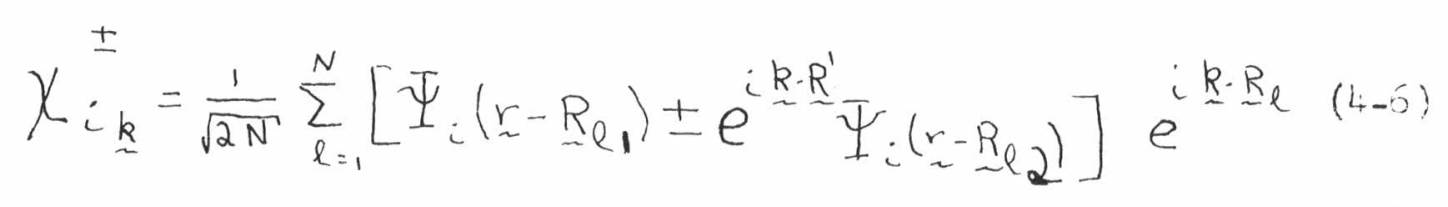

where $\underline{R}_{l 1}=\underline{R}_{e} ; R_{l 2}=R_{l}+\underline{R}^{\prime}$.

\section{b. Energies}

The interactions are also translationally invariant and to find the energies of $(1+6)$ with respect to $(4-3)$, we calculate, in first order,

$$
\varepsilon_{i \underline{\underline{k}}}^{ \pm}=\left\langle\chi_{i \underline{\underline{k}}}^{ \pm}|\boldsymbol{H}| \chi_{i \underline{\underline{k}}}^{ \pm}\right\rangle-\left\langle\Psi_{0}\left|\psi_{\mid}\right| \Psi_{0}\right\rangle
$$

where

$$
A=H_{0}+G \equiv H_{0}+\sum_{I>J} \frac{e^{2}}{R_{I J}}+\sum_{i>j} \frac{e^{2}}{r_{i j}}-\sum_{I, j}^{\prime} \frac{e^{2}}{r_{I j}}
$$


Considering only two-body central forces between molecules and, in the spirit of our molecular wave functions, neglecting intermolecular differential overlap we finally obtain after tedious but straight forward algebra: $47,49,50$

$$
\varepsilon_{i k}^{ \pm}=E_{i}-E_{0}+D_{i}+\Delta_{i}(\underline{\sim}) \pm d_{i}(\underline{R})
$$

where

$$
\begin{aligned}
& D_{i}=\sum_{l=1}^{N} \sum_{\alpha=1}^{2}<\Phi_{i}\left(r_{1}\right) \Phi_{0}\left(r_{\tau^{2}}-R_{l l \alpha}\right)\left|G_{12}\right| \Phi_{i}\left(r_{1}\right) \Phi_{0}\left(r_{2}-R_{-l \alpha}\right) \\
& -<\Phi_{0}\left(r_{1}\right) \Phi_{0}\left(r_{2}-R_{l \alpha}\right)\left|G_{12}\right| \Phi_{0}\left(r_{1}\right) \Phi_{0}\left(r_{2}-R_{l \alpha}\right) \text {, } \\
& \Delta_{i}=\sum_{l=1}^{N}\left\langle\Phi_{i}\left(r_{i}\right) \Phi_{0}\left(r_{\sim^{2}}-R_{l}\right)\left|G_{12}\right| \Phi_{0}\left(r_{i}\right) \Phi_{i}\left(r_{2}-R_{l}\right)\right\rangle \cos \underset{\sim}{R} \cdot \underset{\sim}{R} \underset{l}{(4-10)} \\
& d_{i}=\sum_{l=0}^{N}\left\langle\Phi_{i}\left(r_{i}\right) \Phi_{0}\left(r_{2}-R_{l 2}\right)\left|G_{12}\right| \Phi_{0}\left(r_{1}\right) \Phi_{i}\left(r_{2}-R_{l l}\right)\right\rangle \cos \underset{\sim}{k} \cdot \underset{i l 2}{R_{l}},
\end{aligned}
$$

where $R_{0} \equiv 0, G_{12}$ is the expression in (4-8) for two molecules each with one electron separated by the appropriate $\underset{\sim}{\mathrm{R}}$.

Thus, the excitation energy of the crystal is given by the excitation energy of the molecule shifted by the coulomb energy due to inter-molecular interactions (D), a $k$ dependent term which diagonalizes the transfer of excitation between equivalent molecules via the coulomb interaction $(\Delta)$, and finally the Davydov splitting 48 (d) which diagonalizes the transfer of excitation between inequivalent molecules via the coulomb interaction.

The expressions in equations $(4-6),(4-7),(4-9)$ and $(4-10)$ give the energy shifts and splittings in first order of perturbation theory. If it is necessary to extend the calculation to the second 
order of perturbation theory, we must modify $(4-6)$, the wave functions, by allowing $G$, in (4-8), to mix the zero order states. Further, the energy is modified by adding to $(4-10)$ the second order corrections which are expressions identical to those in (4-10) after replacing

$$
G_{12} \text { by } \sum_{m, n} \frac{G_{12}|m n\rangle\langle m n| G_{12}}{E_{m}+E_{n}-E_{i_{0}}}
$$

where $m$ and $n$ run, individually, through the complete set of molecular states. If $\mathrm{G}_{12}$ is expanded in a multipole series, the first contribution of $\left(I_{+}-11\right)$ to the energy is the Van der Waals interaction.

For odd parity states, the splittings, oscillator strengths, polarization ratios and $\underline{k}$ dependences of the energy have been discussed extensively in the work of Davydov 48 and more recently, among others, by Rice \& coworkers at Chicago. 49 We shall have further reference to the Chicago work later. The state studied most extensively in anthracene is the lowest energy transition. Experimentally, the shift in energy from the gas to the solid is $0.12 \mathrm{eV}$ to the red, the Davydov splitting is $0.4 \mathrm{eV}$ and the polarization ratio $P\left(f_{b} / f_{a}\right)=7.53$ Theoretically, the absolute shift has not been calculated to the author's knowledge; the contributions to the shift, in the multipole expansion, are mainly due to the quadrupolequadrupole and Van der Waals interactions in D, and the dipole-dipole interactions in $\Delta$. The Davydov splitting has been calculated with ambiguous agreement with experiment. This splitting calculation by 
the Chicago group 49 showed that exchange contributions to the energy were small, that the dipole-dipole interaction was dominant outside a sphere of radius $50 \AA$, and that other multipole terms and second order contributions were important within the sphere. This paper calculated all matrix elements using Pariser's 27 wave functions. However, the theoretical calculations were compared with experiments giving an oscillator strength of 0.1 and a polarization ratio of 5 . Finding that the calculation predicted an oscillator strength of 0.4 , the matrix elements were scaled to fit the lower oscillator strength. The recent experimental work of Brodin and Marisova 53 showed that Pariser's wave functions did give the experimental oscillator strength. Therefore scaling calculations using Pariser's wave functions to obtain agreement with experiment is unnecessary. This experimental work suggests a reexamination of the calculated results of the Chicago group, rather than the scaled results which they discuss. On the other hand, their discussion of the relative importance of terms is unaffected, and we shall draw on it. Finally, the measured polarization ratio is quite close to that calculated from the oriented gas model and we shall interpret this as suggesting that the second order corrections to the wave functions are small. Hence, the zero order wave functions will be used in computing transition probabilities.

We pass to even parity states. No calculations have yet been made for even parity exciton states. Two-photon absorption by the crystal and the two-photon spectroscopy experiments of Frohiich and Mahr, ${ }^{55}$ reported recently, raise the possibility that even parity 
exciton states may have been observed directly and thus make the computation of the energies of these states timely. The calculations which follow are intended as an initial rather than an exhaustive treatment of the energies of even parity states in anthracene.

We neglect overlap and work in the approximation of zero differential overlap since exchange effects are small. 49 Further, we shall calculate only the lowest non-vanishing multipole interaction in first order of perturbation theory and the maximum effect of the Van der Waals interaction in second order. We shall reduce this maximum value of the Van der Waals interaction by choosing a reasonable average energy and coupling this with the first order effect compare the results with available experiments. Accuracy within a factor of two, in the shifts and the splittings independently, is the most that can be expected from this estimate.

Summarizing the results which follow, it is concluded that the absolute energy shifts are mainly due to the Van der Waals interaction. The splittings and $\underline{k}$ dependence, however, are mainly due to first order corrections. The levels at $4.94 \mathrm{eV}\left(B_{1 \mathrm{~g}}\right), 5.00$ and $6.81 \mathrm{eV}$ (Alg) in the molecule, remain in the same order in the crystal, while all of them are depressed. The $A_{g}$ and $B_{g}$ states derived from the $B_{I g}$ state of the molecule are the only possible candidates for comparison with Frohlich and Mahr's observations.

Anthracene crystallizes in a monoclinic structure with primitive transiation vectors $\underline{a}, \underline{b}$, and $\underline{c}$ where the first two are mutually perpendicular. We construct a third vector $\hat{c}^{\prime}$ perpendicular to $\hat{a}$ and $\hat{b}$ (see Fig. $4-1$ ) and refer to this set as the cell basis 


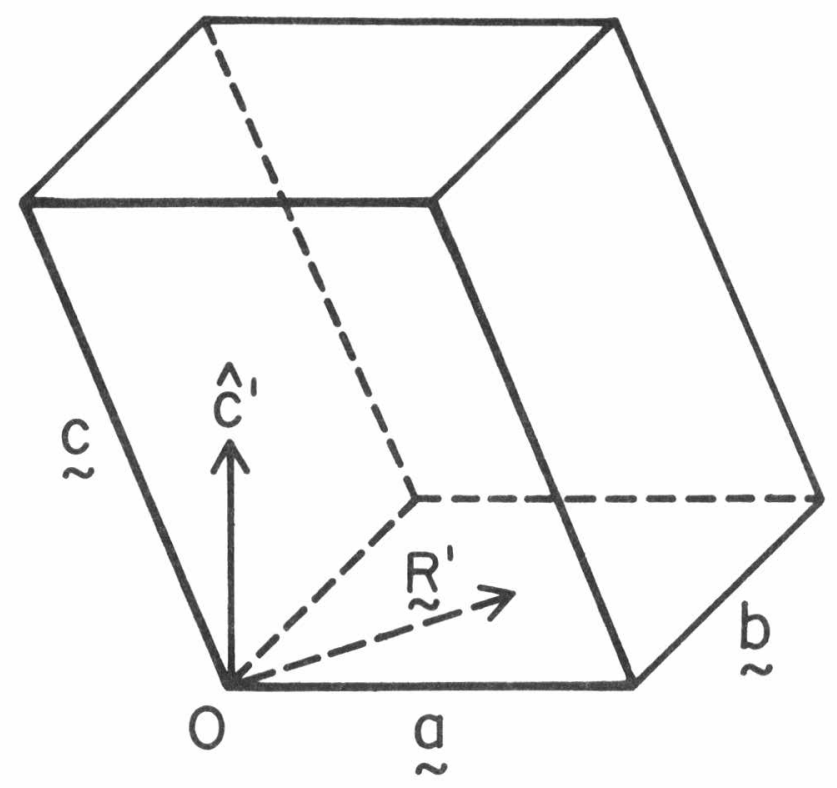

$$
\begin{aligned}
& |\underset{\sim}{a}|=16.2 a_{0} \hat{a} \\
& |\underset{\sim}{b}|=11.4 a_{0} \hat{b} \\
& |\underset{\sim}{c}|=21.1 a_{0} \hat{c}
\end{aligned}
$$$$
\underset{\sim}{a} \cdot \underset{\sim}{b}=0, \underset{\sim}{a} \cdot \underset{\sim}{c}=|\underset{\sim}{a}||\underset{\sim}{c}| \cos 124.7^{\circ}
$$

Molecule I at O; Molecule 2 at $1 / 2(\underset{\sim}{a}+\underset{\sim}{b})$

$$
\underset{\sim}{R^{\prime}}=1 / 2(\underset{\sim}{a}+\underset{\sim}{b})
$$

Fig. 4-I 
set. The orientation of the molecules in the unit cell is given in Figure 4-2. The basis set of the molecule is $\hat{x}_{m}, \hat{y}_{m}$, and $\hat{z}_{m}$ as the long, short and normal molecular axes respectively. The molecular and cell bases are related by Figure 4-2, or in matrix notation, (Euler rotations) $)^{52}$

$$
\left(\begin{array}{l}
\hat{a} \\
\hat{b} \\
\hat{c}^{\prime}
\end{array}\right)=\left(\begin{array}{lll}
-.4950 & +.3207 & \mp .8072 \\
\mp .1222 & \pm .8943 & +.4301 \\
+.8602 & +.3118 & \mp .4034
\end{array}\right)\left(\begin{array}{l}
\hat{x}_{m} \\
\hat{y}_{m} \\
\hat{z}_{m}
\end{array}\right)(4-12)
$$

The upper signs refer to the molecule centered at $O$ in the unit cell (molecule 1), and the lower signs to the other molecule, (molecule 2). The lattice can be described, from 0 , by

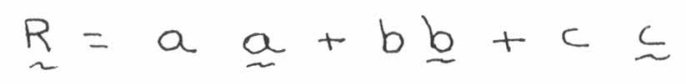

and

$$
\underline{R}+\underline{R}^{\prime}=\left(a+\frac{1}{2}\right) \underline{a}+\left(b+\frac{1}{2}\right) \underline{b}+c \approx
$$

where $a, b$, and $c$ are integers and $\underline{P}^{\prime}$ is the vector between the two molecules in the unit cell. Appendix D specifies the positions of all the molecules within $50 \mathrm{a}_{\mathrm{O}}$ of $\mathrm{O}$, which is as far as the sums were carried.

Defining the interaction coordinate system of two molecules, one at $O$ and the other at $\underline{R}$, as having $\hat{z}_{R}$ in the direction of $\underline{R}$ with $\hat{x}_{R}$ and $\hat{y}_{R}$ conveniently chosen, we can relate it to the cell coordinate system by

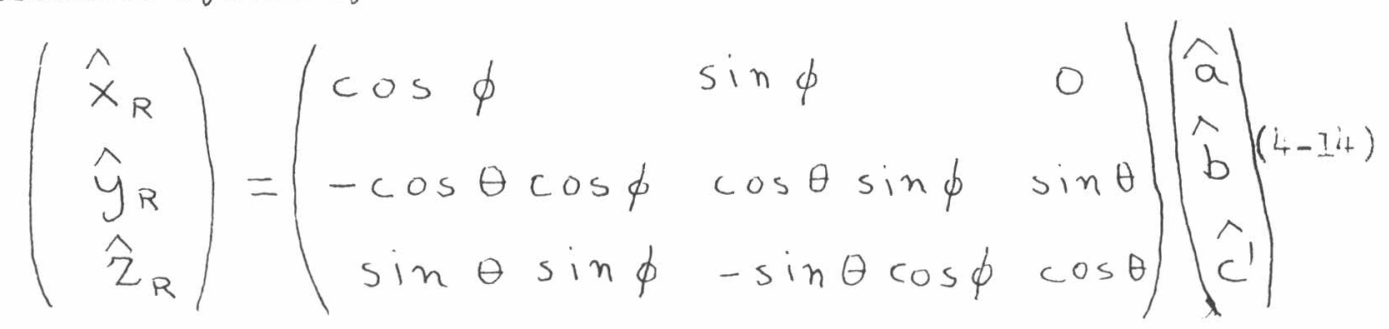

where, for equivalent molecules, 


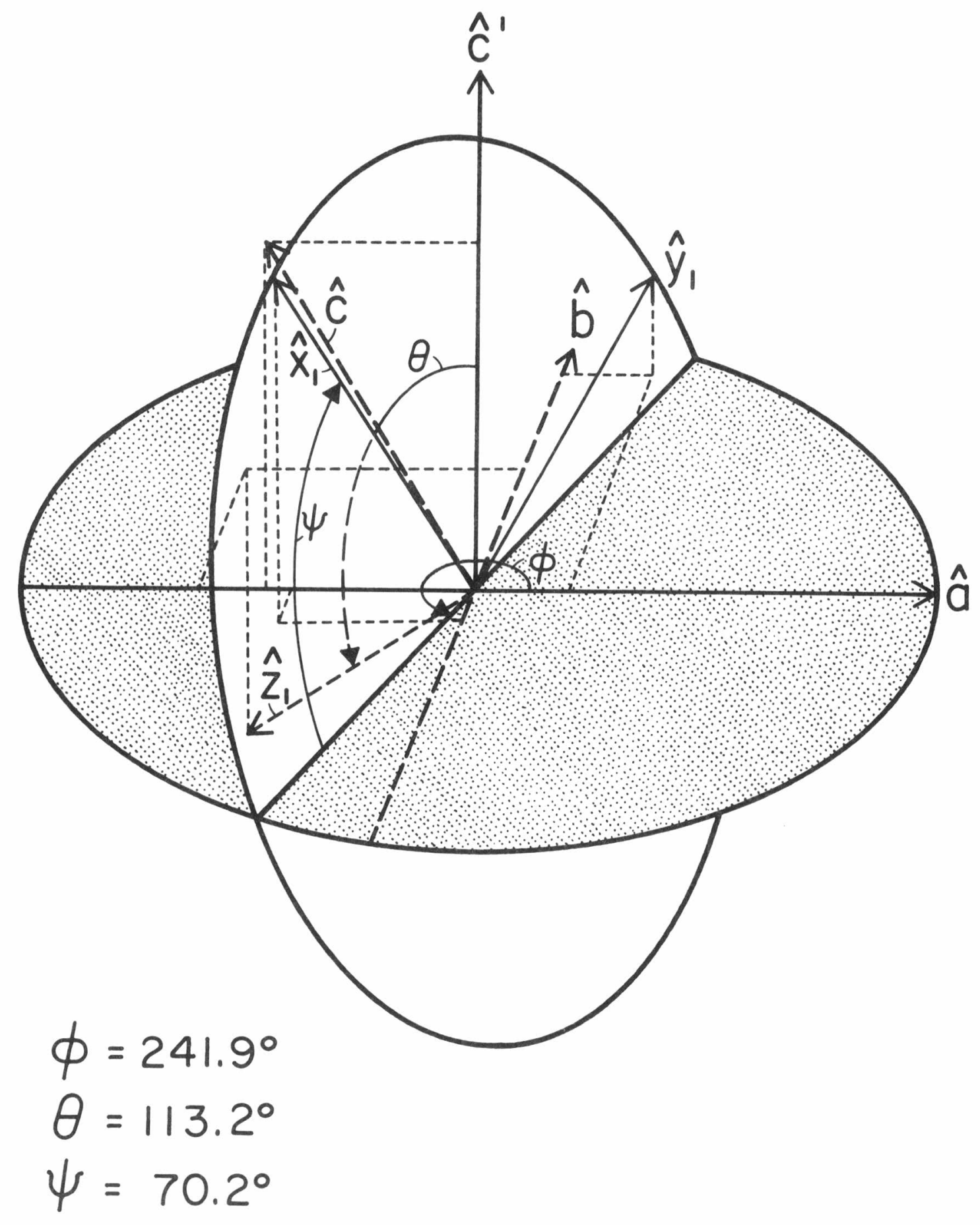

Fig. 4-2 


$$
\begin{gathered}
\cos \theta=\frac{\mid 7.3 c}{|\underline{R}|} ; \sin \theta=\frac{S}{|\underline{R}|} \\
\cos \phi=-\frac{11.4 b}{S} ; \sin \phi=\frac{\mid 6.2 a-12.0 c}{S(4-15)} \\
S=\sqrt{\mid \underline{|R|^{2}-(17.3 c)^{2}}}
\end{gathered}
$$

(all distances in $\left.a_{0}\right)$; while, for inequivalent molecules we use $(4-14)$ and $(4-15)$ with $a \longrightarrow a+1 / 2, b \rightarrow b+1 / 2$, and $|\underline{R}| \rightarrow|\underline{R}+\underline{R}|$. Matrix elements in the interaction coordinate system can be related to those in the molecular basis set by using (4-12)-(4-15).

Expanding the interaction given in $(4-8)$ in a multipole series, the first non-vanishing interaction in first order of perturbation theory is the quadrupole-quadrupole interaction. The nonvanishing terms are given, in the interaction coordinate system, by

$$
\begin{aligned}
G_{12}=\frac{3 e^{2}}{4 R^{5}} & {\left[r_{1}^{2}\left(3 r_{2}^{2}-7 z_{2}^{2}-2 x_{2}^{2}\right)+z_{1}^{2}\left(-7 r_{2}^{2}+19 z_{2}^{2}+2 x_{2}^{2}\right)\right.} \\
& +2 x_{1}^{2}\left(-r_{2}^{2}+z_{2}^{2}+2 x_{2}^{2}\right)-16 x_{1} z_{1} x_{2} z_{2}^{2} \\
& \left.-16 y_{1} z_{1} y_{2} z_{2}+4 x_{1} y_{1} x_{2} y_{2}\right] .
\end{aligned}
$$

Using the wave functions in Appendix A we calculate the matrix elements of Table 4-1 which are detailed in Appendix $C$. The sums in (4-10) converge rapidly with vanishing contributions for molecules separated by more than $40 a_{0}$. The coulomb energy (D) for the 4.9 , 5.0 and $6.8 \mathrm{eV}$ states is shown in Figures $4-3,4$ and 5 respectively. We plot the interaction sums within spheres of increasing radius, the energies are given in $\mathrm{eV}$. The $\underline{k}$ dependent energy shift and splitting ( $\Delta$ and $d$ ) at $k=0$ are shown in Figures $4 . .6,7$, and 8 for 
TABLE $4-1$

The integrals needed for the energy shift and two-photon transition probabilities are:

\begin{tabular}{ccccccccc} 
& \multicolumn{5}{c}{$\Phi_{E_{1}} F_{m} \Phi_{E_{2}} d_{\sim}$} & $\left(i n\right.$ units of $\left.a_{0}^{2}\right)$ \\
$F_{m}$ & $0-0$ & $0-5$ & $0-6.8$ & $0-4.9$ & $5-5$ & $6.8-6.8$ & $4.9-4.9$ \\
$z^{2}$ & 6.23 & 0 & 0 & 0 & 6.23 & 6.23 & 6.23 \\
$x^{2}$ & 26.48 & -1.22 & 16.87 & 0 & 28.79 & 75.02 & 76.43 \\
$y^{2}$ & 12.92 & 1.03 & -2.21 & 0 & 16.47 & 18.28 & 14.89 \\
$x y$ & 0 & 0 & 0 & 7.79 & 0 & 0 & 0
\end{tabular}





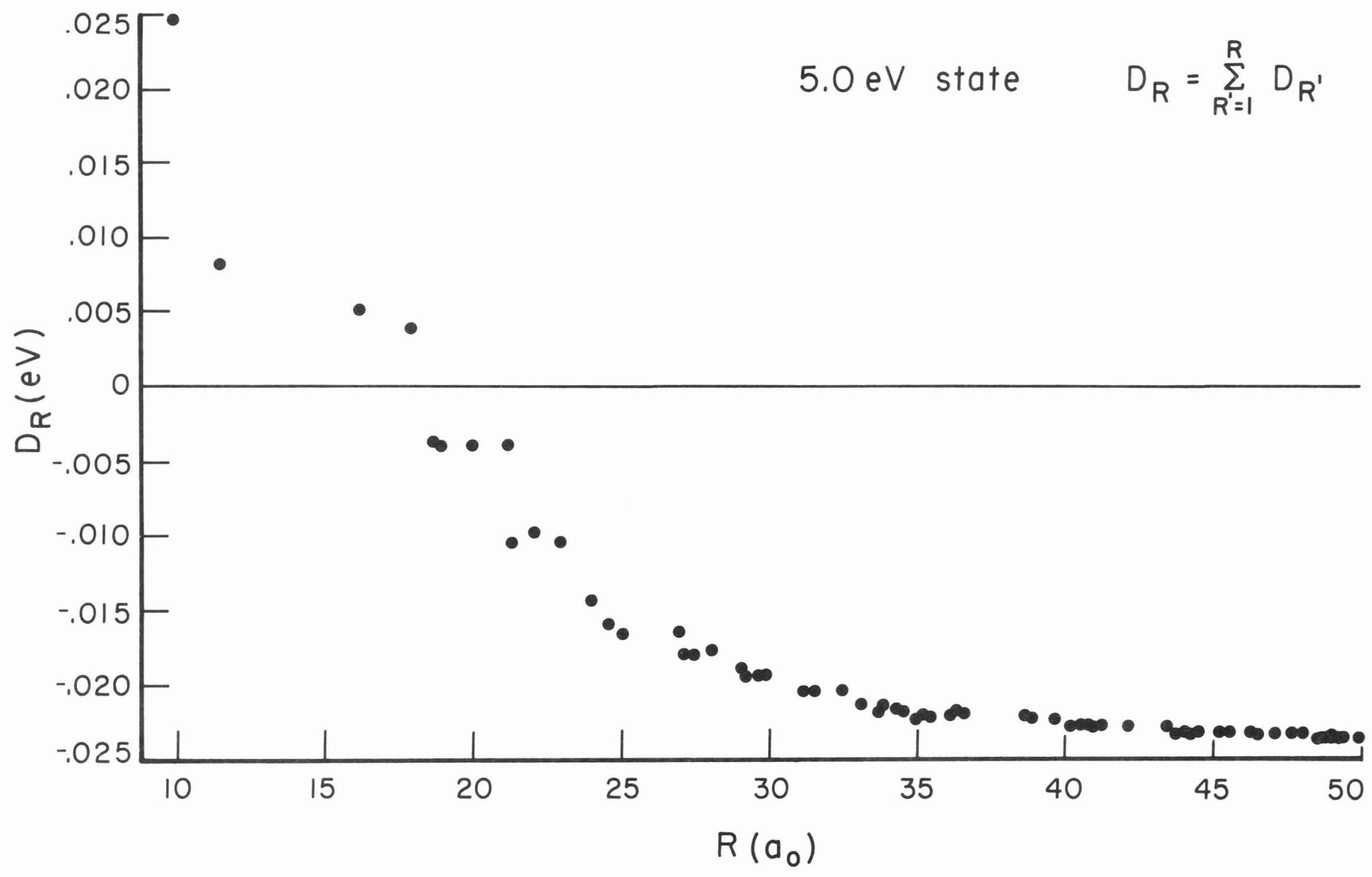

Fig. 4-4 


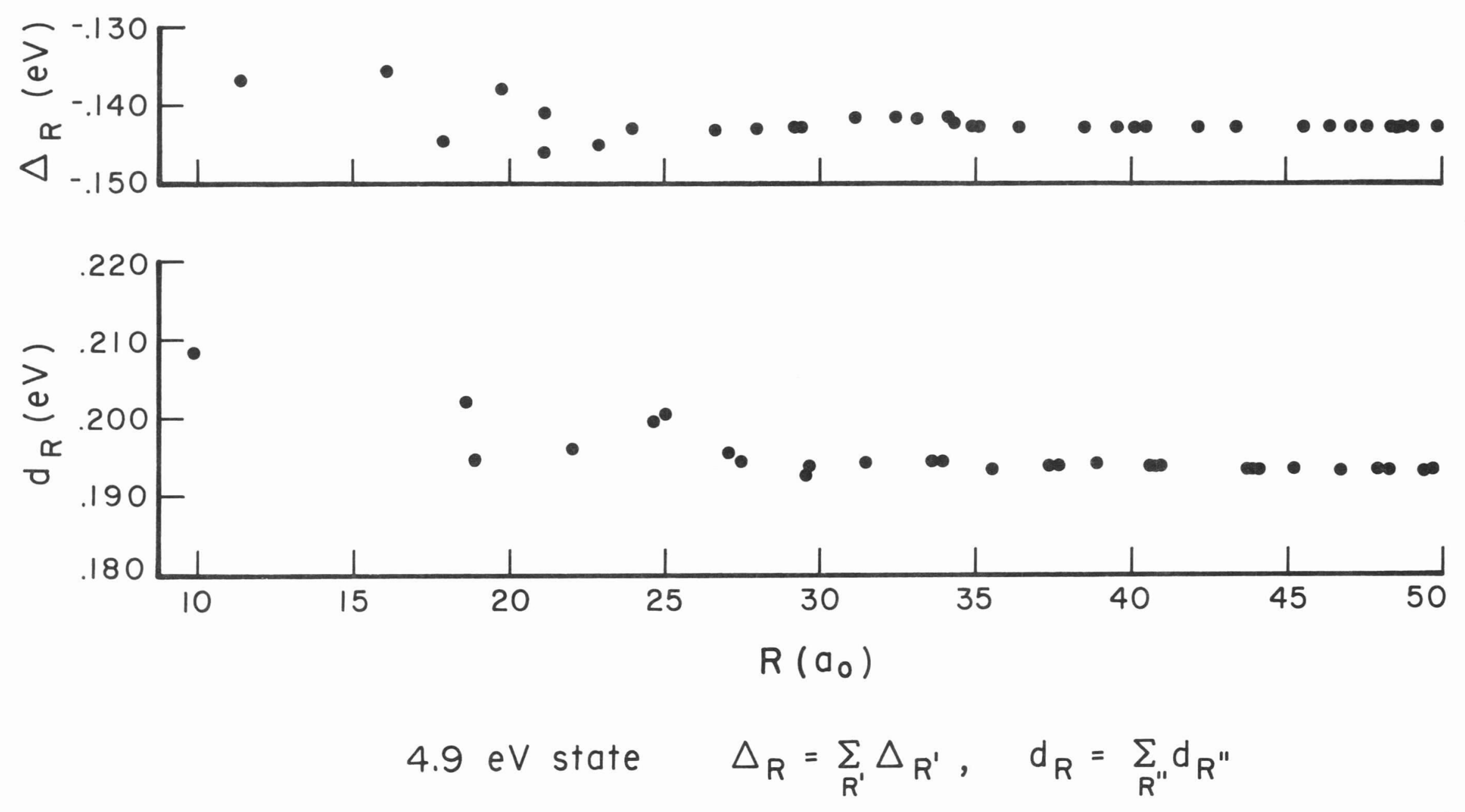

Fig. 4-6 


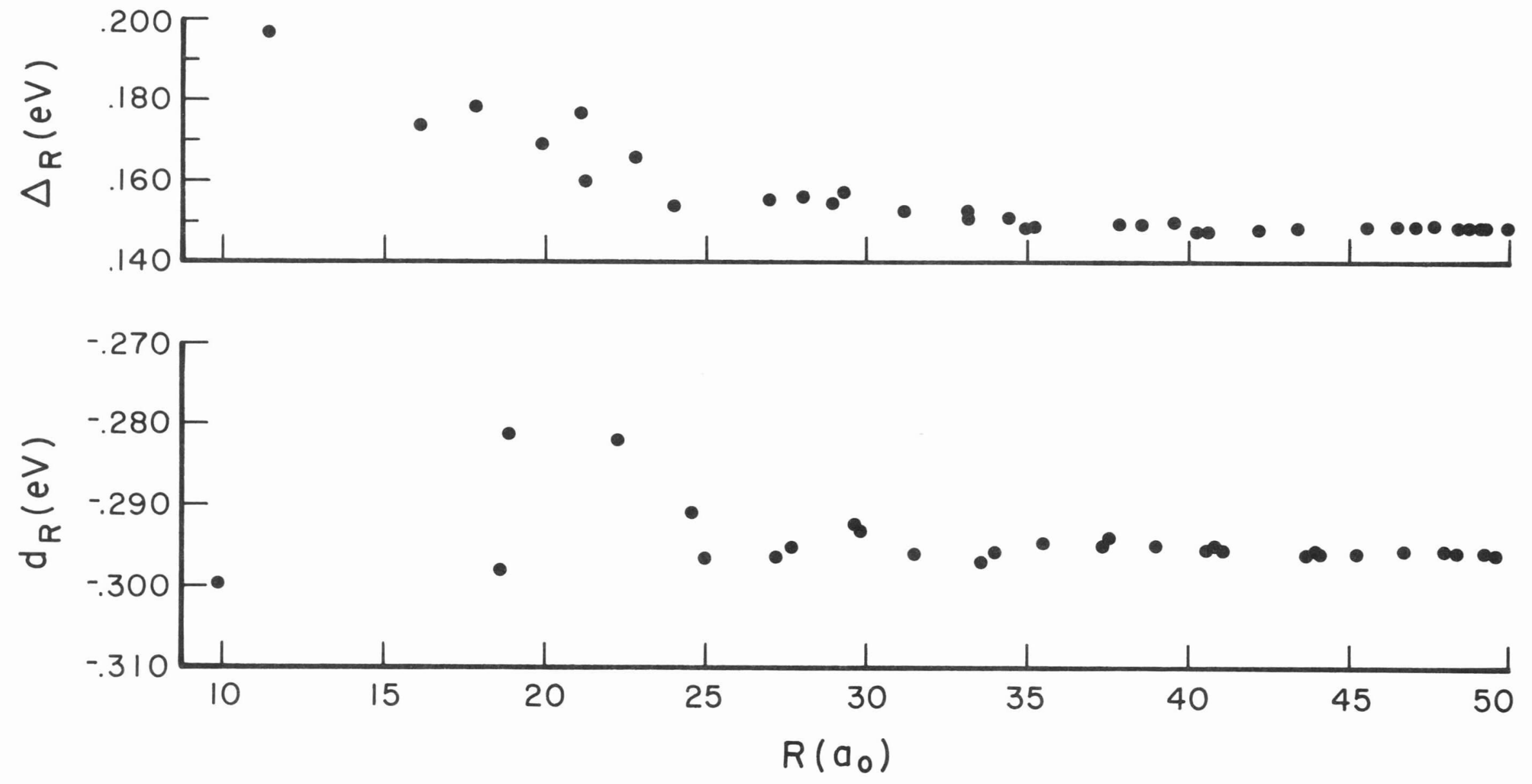

$6.81 \mathrm{eV}$ state $\Delta_{R}=\sum_{R^{\prime}} \Delta_{R^{\prime}}, \quad d_{R}=\sum_{R^{\prime \prime}} d_{R^{\prime \prime}}$

Fig. 4-8 
the three molecular states. We plot the partial sums in eV vs sphere radius in $a_{0}$. Figure $4-9$ summarizes the results of the first order calculation with only the quadrupole-quadrupole (Q-Q ) term. The experimentally observed odd parity states are shown for comparison. 49,53 These results shall be discussed after second order contributions are evaluated.

The first non-vanishing contribution in second order of perturbation theory is the Van der Waals interaction, in interaction coordinates:

$$
\begin{gathered}
G_{12}=x_{1} x_{2}+y_{1} y_{2}-2 z_{1} z_{2} \\
D_{v d w}^{i}=\sum_{l=1}^{N} \sum_{\alpha=1}^{2} \sum_{m, n} \frac{e^{4}}{R_{l \alpha}^{6}} \frac{\left|\left\langle\Phi_{i}\left(r_{1}\right) \Phi_{0}\left(r_{2}-R_{l \alpha}\right)\left|G_{12}\right| \Phi_{m}\left(r_{-1}\right) \Phi_{n}\left(r_{-2}-R_{l \alpha}\right)\right\rangle\right|^{2}(4-17)}{E_{m}-E_{n}-E_{i_{0}}}
\end{gathered}
$$

The expressions for $d$ and $\Delta$ are obtained by comparing equations $(4-10)$ and $(4-17)$. The maximum contribution of the Van der Waals interaction can be calculated by weighing each term of the sum by the smallest denominator, instead of its correct denominator. Maximizing (4-17) in this manner we obtain

$$
D_{v d W M}^{i}=\frac{e^{4}}{E_{i}-2(3.28 \mathrm{a} V)} \sum_{l=1}^{N} \sum_{\alpha=1}^{2} \frac{1}{R_{l \alpha}^{6}}\left\langle\Phi_{i}\left(r_{i}\right) \Phi_{0}\left(r_{2}-R_{l \alpha}\right)\left|G_{12}^{2}\right| \Phi_{i}\left(r_{1}\right) \Phi_{0}\left(r_{2}-R_{l \alpha}\right)\right\rangle(4-18)
$$

with similar expressions for $\Delta$ and $d$. Using the matrix elements of Table 4-1, once again, we obtain the results of Table 4-2 with the first order results included for comparison.

From Table $4-2$ we can conclude: first, both the $4.9 \mathrm{eV}$ and the $5.0 \mathrm{eV}$ states shift to the red in the solid; second, the shifts 
GAS

SOLID, Q-Q CALC.

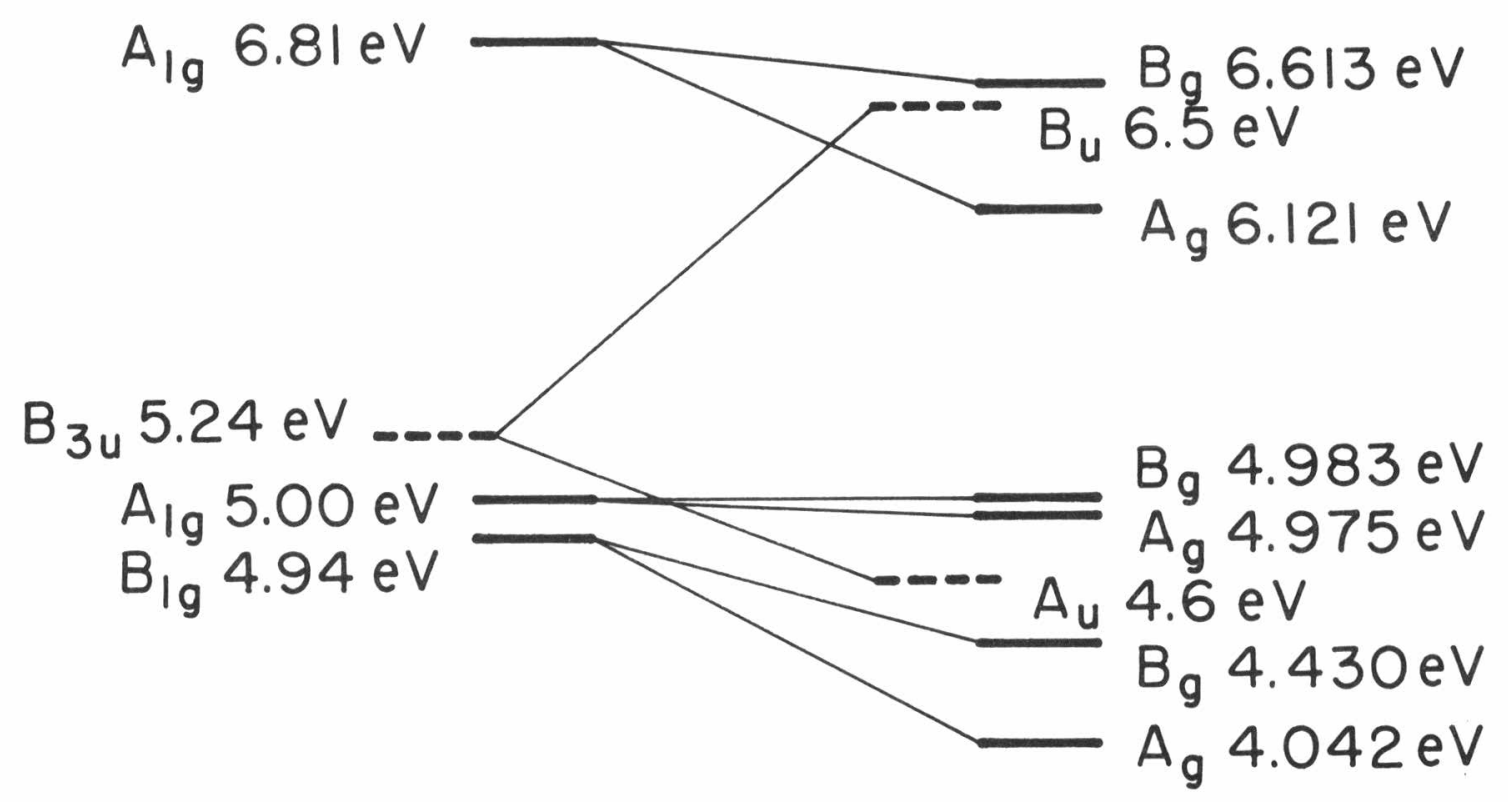

$B_{2 u} 3.28 \mathrm{eV}=-D===\begin{aligned} & B_{u} 3.153 \mathrm{eV} \\ & A_{u} 3.125 \mathrm{eV}\end{aligned}$
$A_{1 g} O$ eV
$-\mathrm{Ag}_{g} \mathrm{OeV}$

Fig, 4-9 
TABLE 4-2

Maximum Van der Waals Interaction (in $\mathrm{eV}$ )

\begin{tabular}{|c|c|c|c|c|}
\hline & ate & $4.94 \mathrm{eV}$ & $5.00 \mathrm{eV}$ & Ground State \\
\hline D & & -2.971 & -1.054 & -.221 \\
\hline$\triangle$ & $(k=0)$ & +.058 & -.001 & \\
\hline d & $(k=0)$ & -.179 & +.0009 & \\
\hline
\end{tabular}

$\mathrm{Q}-\mathrm{Q}$, First Order (in eV)

\begin{tabular}{|c|c|c|c|c|}
\hline \multicolumn{2}{|c|}{ State } & $4.94 \mathrm{eV}$ & $5.00 \mathrm{eV}$ & Ground State \\
\hline D & & -.565 & -.023 & 0 \\
\hline$\Delta$ & $(k=0)$ & -.142 & $+.00 ?$ & \\
\hline d & $(k=0)$ & +.194 & -.004 & \\
\hline
\end{tabular}


are dominated by the Van der Waals interaction; third, the $\underline{k}$ dependence is dominated by the first order interaction; finally, the $\mathrm{B}_{\mathrm{g}}$ state is higher in energy in both cases. The result of the maximum Van der Waals interaction plus the quadruple-quadrupole interaction are summarized in Figure 4-10.

The wave vector dependence of the bands derived from the 4.9 and $5.0 \mathrm{eV}$ states respectively are given in Appendix E. This Appendix shows the quadrupole-quadrupole first order calculation and the maximum Van der Waals contribution. The energies are in milli-eV, $\mathrm{k}_{\mathrm{a}}$ is in the direction $\underline{\mathrm{b}} \mathrm{x} \underline{\mathrm{c}}, \mathrm{k}_{\mathrm{b}}$ along $\underline{c} \mathrm{x} \underline{\mathrm{a}}$ and $\mathrm{k}_{\mathrm{c}}$ along $\underline{\mathrm{a}} \mathrm{x} \underline{\mathrm{b}}$. The edges of the zone are respectively at $\pi / \mathrm{a}$ with $\mathrm{a}=16.2 \mathrm{a}_{0}, \pi / \mathrm{b}$ with $b=11.4 a_{0}$ and $\pi / c$ with $c=21.1 a_{0}$.

Previous experience with the Van der Wals interaction allows a more reasonable estimate once the maximum contribution has been calculated. Using the average energy technique we can estimate the Van der Waals contribution. This technique is also used in polarizability calculations where it is found that the choice of an average energy at about twice the ionization potential gives reasonable results. 34 Let us estimate a somewhat lower average energy, twice $6 \mathrm{eV}$ for anthracene. This choice yields a contribution to the binding energy from the van der Waals term of $0.12 \mathrm{eV}$ per molecule which is the typical contribution for the rare gas solids as well. This assumption gives the results summarized in Figure 4-11 for $Q-Q$ plus "reasonable $\mathrm{V} d \mathrm{~W}$, "(the ground state is moved up to the zero of energy as a datum).

Frohlich and Mahr observe absorption series starting at 3.48 
Gas

$$
\begin{array}{r}
\frac{Q-Q+\text { Maximum }}{\text { Van der Waals, Solid }} \\
\hline
\end{array}
$$

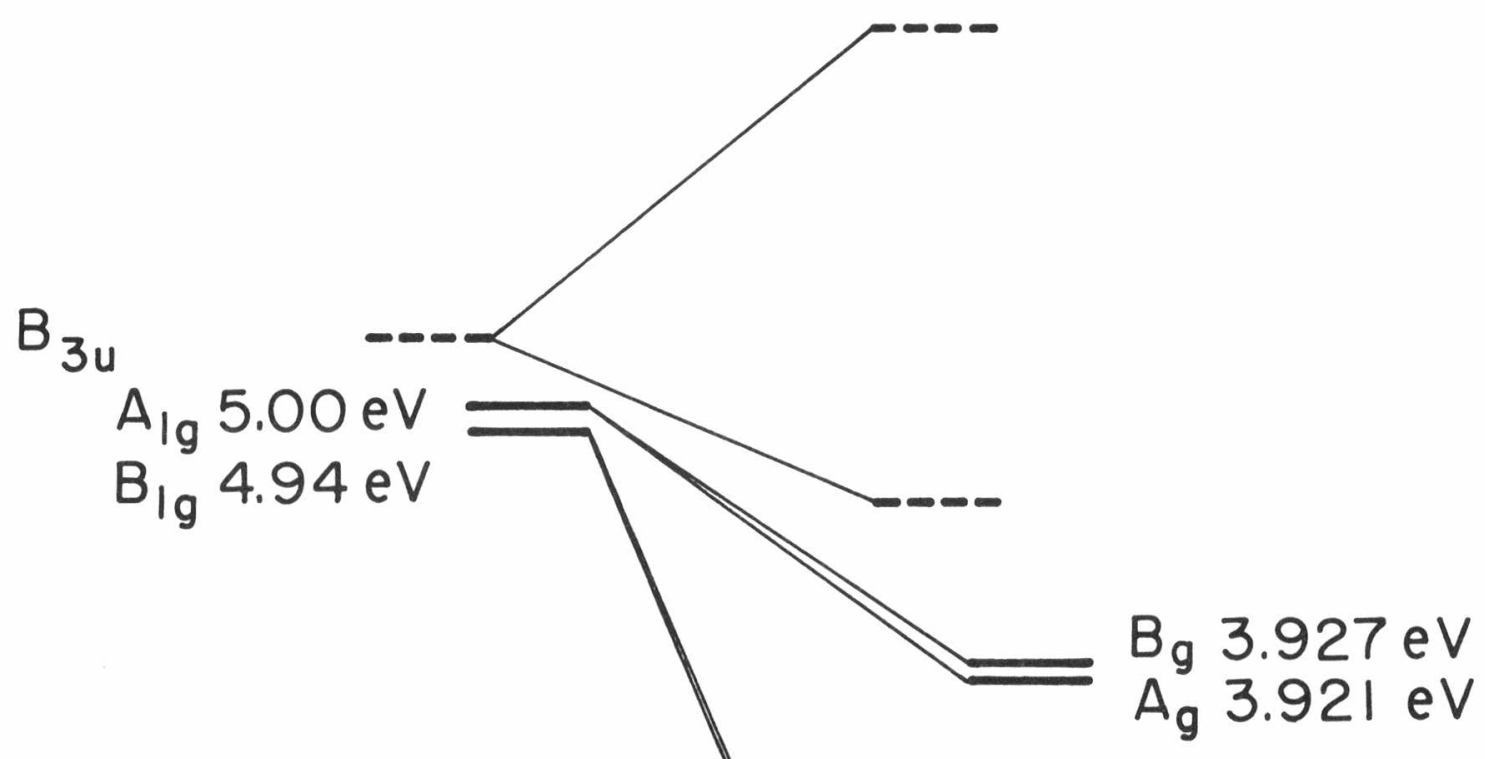

$B_{2 u}$

$$
A_{1 g} O \text { eV }
$$

$$
A_{g}-.221 \mathrm{eV}
$$

Fig, 4-10 
Gas

Q-Q + "Reasonable"

Van der Waals, Solid

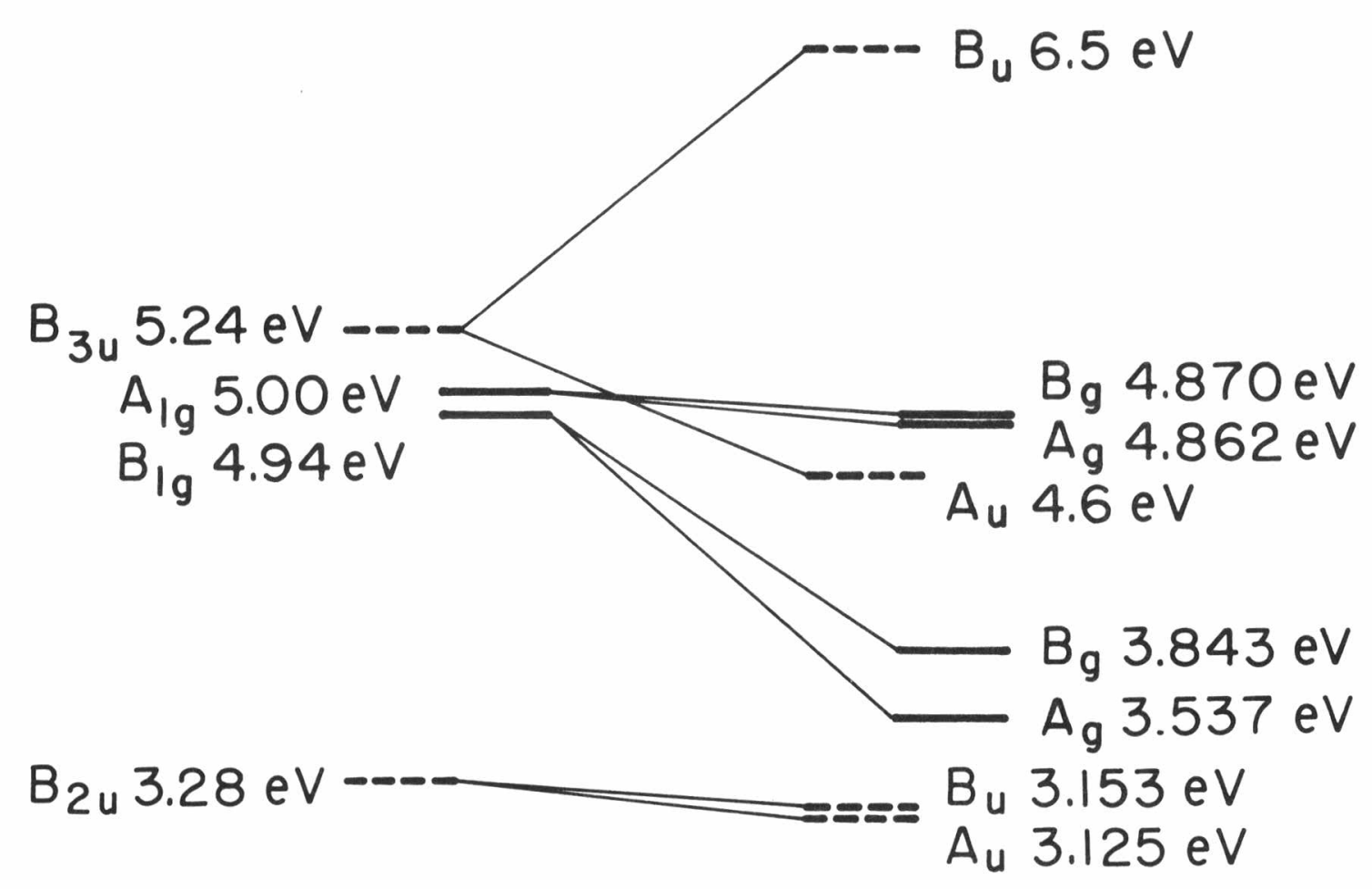
$A_{\text {Ig }} O \mathrm{eV}$
$-A_{g} O e V$

Fig. $4-11$ 
and $3.58 \mathrm{eV}$ which we identify with the $\mathrm{A}_{\mathrm{g}}$ and $\mathrm{B}_{\mathrm{g}}$ states arising from the $B_{1 g}$ molecular state at $4.94 \mathrm{eV}$.

Several questions must be answered before accepting the identification of the observation with our $B_{1 g}$ derived states. In the first place, the angular dependence of the absorption should be measured in detail and compared with our results of the last section of this chapter. These results are consistent with the observations to date, as will be shown, but that is not sufficient. The question of whether the observed states are pure even parity or vibronic mixtures remains. Both of these could have the same angular dependence, and the temperature dependence which would identify a vibronic state might not be observable in the temperature range below the melting point. A possible resolution of the dilemma lies in the absolute strength of the absorption. The vibronic absorption should be about two orders of magnitude weaker than absorption directly by an even parity state. The information presently available indicates that the absorption in the gas and liquid is due to vibronic states since it occurs at energies far below the predicted even parity states. Within the bands observed by Frohlich and Mahr an independent experiment has been performed by McMahon et al ${ }^{56}$ at $3.57 \mathrm{ev}$, they find the absorption in the solid about 70 times larger than that of the liquid at the same energy. This observation lends support to the identification of that solid absorption with even parity electronic states. Then, we would tend to identify the absorption observed in the solid at $3.20 \mathrm{ev}^{19}$ and at $3.16 \mathrm{ev}, 20$ but not observed by Frohlich and Mahr hence weaker, with vibronic states 
due to the mixing of the even parity levels with the $B_{2 u}$ derived levels. All observations must be classified as preliminary data since symmetries are not unambiguously determined, clearly further investigations of the above questions is called for. For vibronic states the energy shifts in going into the solid are dominated by the state which is being perturbed. The contribution of the admixed states to the shifts and the splittings is reduced by the mixing coefficient and thus made small.

\section{Crystal Optics}

Crystals belonging to the orthorombic, monoclinic and triclinic systems are optically biaxial ${ }^{54}$ and therefore double refracting. That is, the index of refraction as a function of direction is a general ellipsoid with the three principal indices having different magnitudes. Further, when light of arbitrary polarization is incident on the crystal it splits into two beams which propagate in different directions. While these beams are unresolved in the crystal the polarization changes character a function of the position. For example, linearly polarized light incident in an arbitrary direction becomes elliptically polarized, going through circular, linear in another direction etc., until, if the crystal is long enoldgh, two linearly polarized beams resolve. Then, that polarization condition remains for all subsequent propagation.

Anthracene crystallizes in the monoclinic system, therefore discussion of its absorption characteristics as a function of polarization must treat the biaxial character of the crystal in order to 
obtain intelligible results.

Consider a crystal cleaved in such a way that the cleavage plane contains one of the principal axes of the index ellipsoid, and let linearly polarized light be incident perpendicular to the cleavage plane. In general, the crystallographic axes need not coincide with the principal axes of the index ellipsoid. For monoclinic crystals one principal axes will coincide with a crystallographic axis due to symmetry. With reference to Figure 4-12, we resolve the polarization of the light as

$$
\hat{\epsilon}=\hat{a} \cos \alpha+\hat{y} \sin \alpha
$$

with

$$
\hat{k}_{\text {inc }} \cdot \hat{y}=\hat{k}_{\text {inc }} \cdot \hat{a}=0 \text {. }
$$

Since there is reflection symmetry across the $x z$ plane, the $y$ polarized component is unrefracted. Constructing Huygen's wavelets it can be seen that, for the a polarized component, the velocity of propagation is anisotropic. The velocity profile in the $x-z$ plane is given by

$$
\frac{v_{x}^{2}}{\left(c / n_{z}\right)^{2}}+\frac{v_{z}^{2}}{\left(c / n_{x}\right)^{2}}=1
$$

The ray direction in the crystal is given by the condition that the tangent to the velocity profile at that point be parallel to $\hat{a}$. This condition is dictated by Maxwell's equations since $\underset{\sim}{D}$ is not changed in crossing the dielectric interface while $\hat{E}=\hat{\epsilon}$ is rotated. This discussion is easily generalized to<smiles>[R]C1C=CC=C1[18O]</smiles>

by treating the component in the $\mathrm{k} \mathrm{y}$ plane in the same way as we 


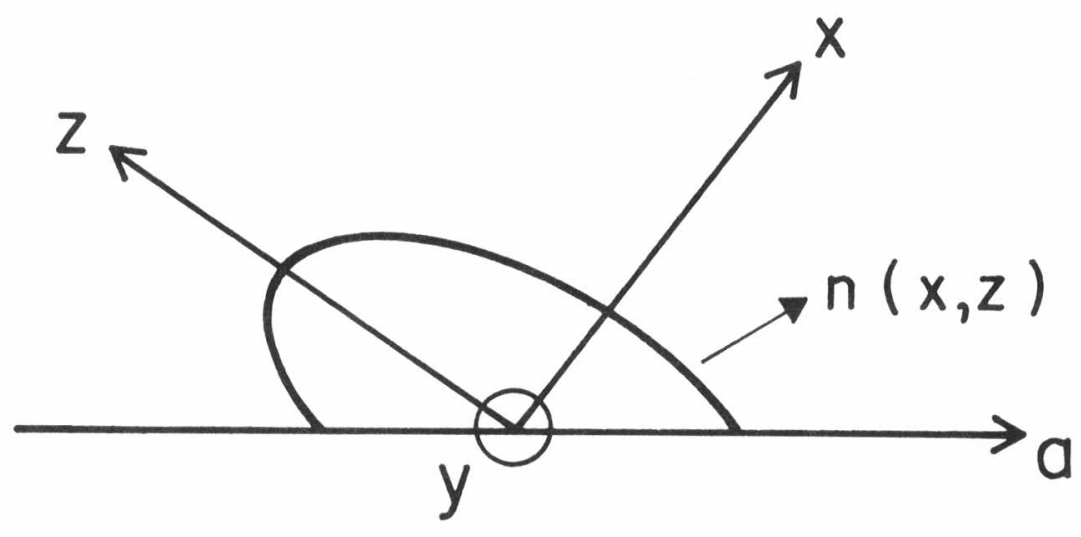

$\uparrow \hat{k}_{\text {incident }}$

$\cos \alpha=\hat{\epsilon} \cdot \hat{a}$

Fig. 4-12 
have treated the $\mathrm{x} z$ component. Both beams would then be refracted. For anthracene the anisotropy of the index has been measured by Nakada ${ }^{45}$ as :

$$
\begin{aligned}
& n_{x}=1.77 \pm .01 \\
& n_{y}=1.55 \pm .01 \\
& n_{z}=2.04 \pm .08
\end{aligned}
$$

(see Fig. 4-13). Therefore the refraction of the a-polarized beam is calculated to $6.2^{\circ} \pm 1.8^{\circ}$ within the accuracy of the index measurements. For crystals where

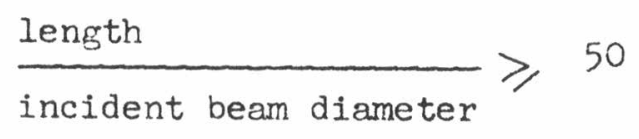

tne two beams are well resolved through most of the absorbing volume and the absorption, including angular dependence and polarization characteristics, can be calculated. We shall treat this, "Iong crystal", case henceforth.

In view of the fact that linearly polarized light (oriented at $\alpha=45^{\circ}$ ) becomes circularly polarized in $\sim 3000 \AA$ it is not surprising that the measurements of Hall et al ${ }^{19}$ did not reveal a strong angular dependence in the absorption of two-ruby photons by anthracene crystals. Further, the experiment of Iannuzzi \& Polacco 22 comparing the absorption due to linear and circular polarized light incident on a powder sample, cannot be meaningful since neither the linear ror the circular polarized light will remain with that polarization after travelling through the chunks of crystal which make up the powder. It is unreasonable to expect that the crystal-chunks in 


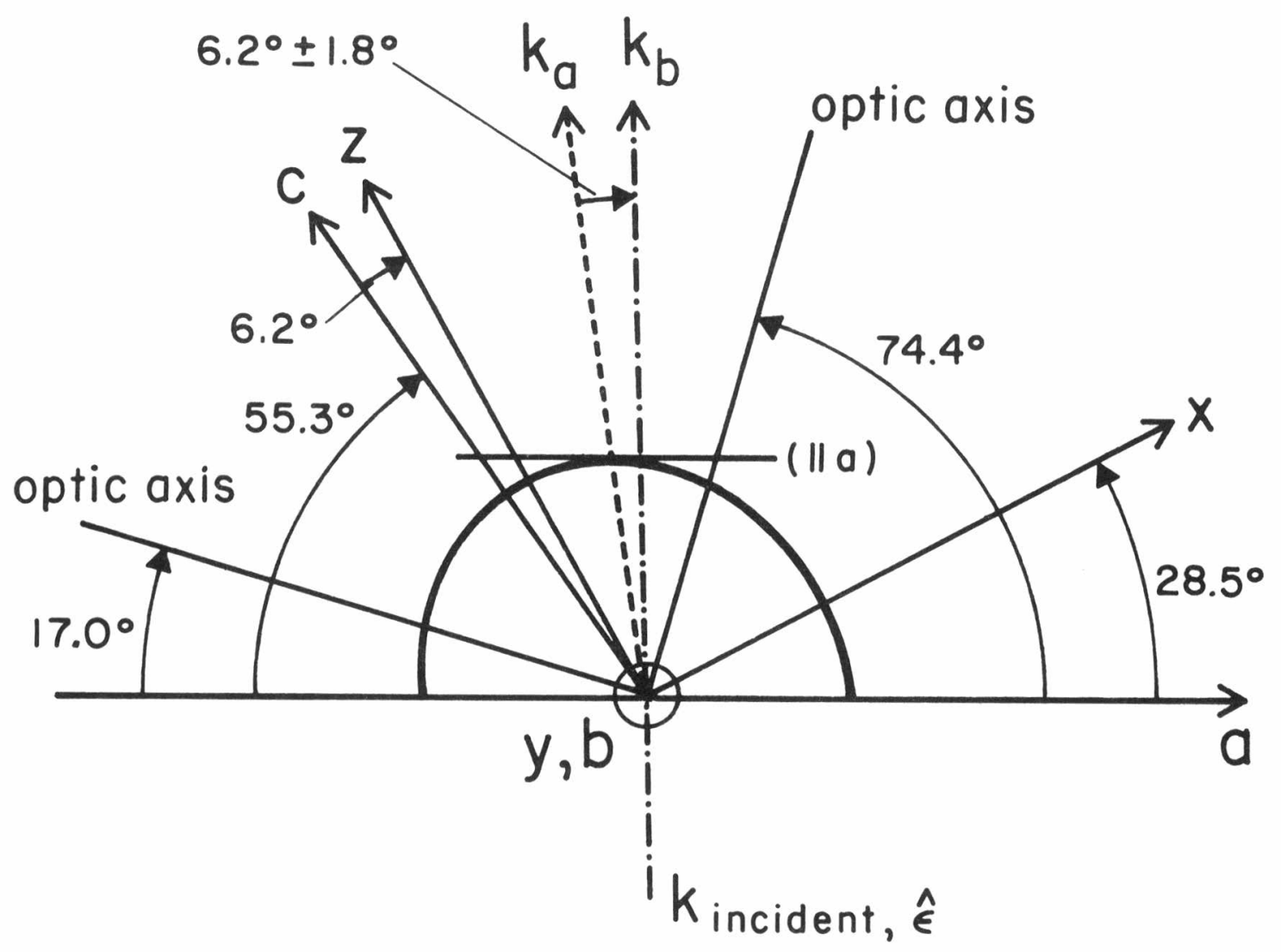

Fig. $4-13$ 
the powder are much smaller than $3000 \AA$ in length.

\section{Two-photon Absorption}

We now treat the two-photon absorption of the states described in the first section of this chapter. We shall draw on the formalism of Chapter 2 and the results of Chapter 3 . We use zeroorder, oriented gas, wave functions for the solid and calculate their absorption.

Using equation (2-11) the transition rate is given by

$$
W_{f i}=C\left(\frac{n_{\omega}\left(\hat{\epsilon}_{1}\right)^{2}+2}{3}\right)^{2}\left(\frac{n_{\omega}\left(\hat{\epsilon}_{2}\right)+2}{3}\right)^{2} \frac{\left|R_{f i}^{(2)}\right|^{2} g\left(2 \hbar_{\omega}\right)}{n_{\omega}\left(\hat{\epsilon}_{1}\right) n_{\omega}\left(\hat{\epsilon}_{2}\right)}
$$

with $\quad C=(2 \pi)^{3}\left(\frac{e^{2}}{\hbar c}\right)^{2} \hbar(\hbar \omega)^{2} F_{1} F_{2}$

For transitions from the ground state, the possible final states are of $\mathrm{A}_{\mathrm{g}}$ and $\mathrm{Bg}$ symmetry derived from the $\mathrm{A}_{\mathrm{Ig}}$ and $\mathrm{B}_{\mathrm{lg}}$ levels of the molecule.

The states of equation $(3-6)$ can easily be classified into the irreducible representations of the group of the crystal, C2r. At $\underline{k} \cdot \underline{R}^{\prime}=0$, the identity and inversion operations in the crystal coincide with those of the molecule, the character table of $\mathrm{C}_{2 h}$ is given in Table 4-3. Using it, we can obtain the transformation properties of the wave functions and these are given in Table $4-4$. Since we shall be concerned, mainly, with the angular dependence of the absorption, we use the resolution of the polarization of the light of equation (4-19). In accordance with the discussion of 
TABLE $4-3$

\begin{tabular}{ccccc}
$\mathrm{C}_{2 h}$ & $E$ & $\mathrm{C}_{2}$ & $J$ & $\mathrm{C}_{2} J$ \\
\hline $\mathrm{A}_{\mathrm{g}}$ & 1 & 1 & 1 & 1 \\
$\mathrm{~B}_{\mathrm{g}}$ & 1 & -1 & 1 & -1 \\
$\mathrm{~A}_{\mathrm{u}}$ & 1 & 1 & -1 & -1 \\
$\mathrm{Bu}_{\mathrm{u}}$ & 1 & -1 & -1 & 1
\end{tabular}


TABLE 4-4

\begin{tabular}{|c|c|c|c|c|c|c|}
\hline in $D_{2 h}$ & & $\mathrm{~A}_{1 \mathrm{~g}}$ & $\mathrm{~B}_{\lg }$ & $B_{2 u}$ & $\mathrm{~B}_{3 \mathrm{u}}$ & Ground state \\
\hline in $\mathrm{C}_{2 h}$ & + & $\mathrm{A}_{\mathrm{g}}$ & $\mathrm{B}_{\mathrm{g}}$ & $\mathrm{B}_{\mathrm{u}}$ & $A_{u}$ & $\mathrm{Ag}_{\mathrm{g}}$ \\
\hline & - & $\mathrm{B}_{\mathrm{g}}$ & $A_{g}$ & $A_{u}$ & $\mathrm{~B}_{u}$ & \\
\hline
\end{tabular}


crystal optics, we find that the polarization vector within the crystal can be written as

$$
\hat{\epsilon}=\sin \alpha \hat{b}+\cos \alpha\left(\cos 6.2^{\circ} \hat{a}+\sin 6.2^{\circ} \hat{c^{\prime}}\right)
$$

where, as previously defined, $\alpha$ is the angle between the polarization of the incident beam and the a crystallographic direction. It should be remembered that we are considering incidence perpendicular to the a b face of the crystal.

Since we know the matrix elements in the molecular coordinate system, it is convenient to write $(4-23)$ in this basis:

$$
\hat{\epsilon}_{\alpha}=l_{\alpha} \hat{x}_{m}+m_{\alpha} \hat{y}_{m}+n_{\alpha} \hat{z}_{m}
$$

where $\alpha$ indicates the basis set of either molecule 1 , in the unit cell, or molecule 2. We can now combine equations (4-23) and (4-12) to obtain the direction cosines in (4-24), take over the discussion of Chapter 3 and write down the angular dependence of absorption.

a. Blg derived states

The transition rate per molecule is

$$
w^{ \pm} \alpha\left|l_{\alpha=1} m_{\alpha=1} \pm l_{\alpha=2} m_{\alpha=2}\right|^{2}
$$

where the direction cosines are obtained from equations (4-23), $(4-24)$ and $(4-12)$. Further, corrections due to the index anisotropy must be included. The most convenient method is to include with each $\sin ^{2} \alpha$ a multiplicative term of $\frac{1}{n_{b}}\left(\frac{n_{b}^{2}+2}{3}\right)^{2}=1.389$ and with each $\cos ^{2} \alpha$ a multiplicative term of $\frac{1}{n_{\perp}}\left(\frac{n_{\perp}^{2}+2}{3}\right)^{2}=1.712 \cdot n_{\perp}$ 
is the index perpendicular to the direction of propagation of the refracted beam. If the refraction angle is taken at $6.2^{\circ}$, as discussed in the previous section, then the appropriate index is 1.81. Performing the indicated algebra we obtain

$$
\begin{aligned}
w_{B_{g}}^{+}=16 C g(2 \hbar \omega)\left|\frac{\left\langle B_{1 g}|x y| A_{1 g}\right\rangle}{\bar{E}_{x y}-\hbar \omega}\right|^{2} \\
\quad \times\left(.023 \sin ^{4} \alpha+.058 \cos ^{4} \alpha+.073 \sin ^{2} \alpha \cos ^{2} \alpha\right)
\end{aligned}
$$

and

$$
W_{A_{g}}^{-}=16 C g(2 \hbar \omega)\left|\frac{<B_{1 g}|x y| A_{1 g}>}{E_{x y}-\hbar \omega}\right|^{2}\left(.38 \sin ^{2} \alpha \cos ^{2} \alpha\right)^{(4-27)}
$$

including the index anisotropy.

The angular dependence is a function of symmetry and geometry alone. That is, the angular dependence predicted above is for all. $B_{1 g}$ derived states. For the specific case of the states derived from the $4.94 \mathrm{eV}$ state in the anthracene molecule, the minus, $\mathrm{Ag}$, state lies lower in energy. When we test the identification of these states with those observed by Frohlich and Mahr ${ }^{55}$ we find consistency in that the lower energy state does not absorb light when both incident beams are polarized along $b(\alpha=\pi / 2)$ while the higher energy state does. Further predictions cannot be tested with their experiments since, for $\alpha \neq \pi / 2$, their laser beam has varying polarization. In their experiment they increased the laser flux in the crystal by internal reflection from the walls of the crystal.

The absolute transition rates are found, as in Chapter 3 , by choosing $\overline{\mathrm{E}}_{\mathrm{XY}}$ as the lowest state of correct symmetry allowed in one photon transitions. Therefore $\bar{E}_{x y}=3.13 \mathrm{eV}$. For the rest of the 
parameters we keep the values chosen in Chapter 3. Then, for the absolute transition rates per molecule we obtain

$$
\begin{aligned}
& W_{B_{g}}^{+}=8.92 \times 10^{-49} F_{1} F_{2}\left(\sin ^{4} \alpha+2.5 \cos ^{4} \alpha+3.2 \sin ^{2} \alpha \cos ^{2} \alpha\right) \sec ^{-1} . \\
& W_{A_{g}}^{-}=1.47 \times 10^{-47} F_{1} F_{2} \sin ^{2} \alpha \cos ^{2} \alpha \quad \sec ^{-1} \text { (4-28) }
\end{aligned}
$$

with $F_{1,2}$ in photons $/ \mathrm{cm}^{2} \mathrm{sec}$.

b. Alg derived states

The transition rate per molecule is parametrized by $\lambda$ as in Chapter 3 , a reasonable $\lambda$ for the solid is

$$
\lambda \equiv \frac{\bar{E}_{x x}-\hbar \omega}{\bar{E}_{y y}-\hbar \omega} \simeq \frac{4.6-\hbar \omega}{3.13-\hbar \omega}=2.1
$$

choosing the lowest $\mathrm{B}_{3 \mathrm{u}}$ derived state at $4.6 \mathrm{eV}$ and the lowest $\mathrm{B}_{2 \mathrm{u}}$ derived state at $3.13 \mathrm{ev}$. Nevertheless we exhibit the results for $\lambda=1,1.5,2,2.5$ and 3.

The transition rate per molecule is given by

$$
w^{ \pm} \alpha\left|\left(l_{\alpha=1}^{2} \pm l_{\alpha=2}^{2}\right)+\left(m_{\alpha=1}^{2} \pm m_{\alpha=2}^{2}\right)(\lambda B)\right|^{2}
$$

Including the index corrections, as discussed previously, and carrying out the algebra we obtain

$$
\begin{array}{rl}
W_{A g}^{+}=4 C & g(2 \hbar \omega)\left|\frac{\left\langle A_{1 g}^{*}\left|x^{2}\right| A_{1 g}\right\rangle}{\bar{E}_{x x}-\hbar \omega}\right|^{2} \\
& x\left(L_{\lambda} \cos ^{4} \alpha+M_{\lambda} \sin ^{4} \alpha+N_{\lambda} \sin ^{2} \alpha \cos ^{2} \alpha\right)
\end{array}
$$

The coeficients, which are functions of $\lambda$, are given in Rable $4-5$.

$$
w_{B_{g}}^{-}=4 c g(2 \hbar \omega)\left|\frac{\left\langle A_{i g}^{*}\left|x^{2}\right| A_{1 g}>\right.}{E_{x x}-\hbar \omega}\right|^{2} Z_{\lambda} \sin ^{2} \alpha \cos ^{2} \alpha \quad(4-32)
$$


TABLE 4-5

\begin{tabular}{|c|c|c|c|c|c|}
\hline$\lambda=$ & 1.0 & 1.5 & 2.0 & 2.5 & $\underline{3.0}$ \\
\hline $\mathrm{I}$ & .058 & .073 & .105 & .153 & .217 \\
\hline M & .84 & 1.92 & 3.44 & 5.40 & 7.79 \\
\hline N & .088 & .38 & .84 & 1.47 & 2.18 \\
\hline Z & .96 & 1.92 & 3.20 & 4.84 & 6.80 \\
\hline
\end{tabular}


The values of $z_{\lambda}$ are given in Table $4-5$ as well. To obtain absolute values of the absorption, we choose $\overline{\mathrm{E}}_{\mathrm{xx}}=4.6 \mathrm{eV}$ and keep the values of Chapter 3 for the rest of the parameters. Then,

$$
4 C\left|\frac{\left\langle A_{1 g}^{*}\left|x^{2}\right| A_{1 g}\right\rangle}{E_{x x}-\hbar \omega}\right|^{2} g(2 \hbar \omega)=5.43 \times 10^{-50} \frac{\mathrm{cm}^{4} \sec }{\text { photon }}(4-33)
$$

which combined with equations $(4-31)$ and (4-32) gives the absolute absorption.

\section{Conclusion}

The $B_{1 g}$ - derived exciton states have a calculated energy and splitting which suggests their identification with the observation of Frohlich and Mahr. ${ }^{5}$ The angular dependence predicted for the absorption is also consistent with the identification of a $\mathrm{B}_{\mathrm{g}}$ state with the transitions observed at $3.58 \mathrm{eV}$ and an $\mathrm{Ag}$ state with those at $3.48 \mathrm{eV}$. The calculated absolute strength of the absorption lends support to this tentative identification. 
CHAPTER V

VIBRONIC STATES

\section{Introduction}

An explanation is presented for the two-photon absorption observed in non-crystalline samples of anthracene and the consequences of this suggestion are explored. Previously we have noted the lack of even parity electronic eigenstates of the molecule at an energy close to twice that of ruby laser photons $(3.57 \mathrm{eV})$, however an odd parity state does exist nearby. In order to make the absorption possible, we introduce an additional interaction in the molecule. This interaction will mix the purely electronic eigenstates and the absorption may take place to a mixed parity final state. Intra-molecular vibrations will accomplish this mixing. These mixed states resulting from the inclusion of vibrations are called vibronic and their properties are discussed.

The results of this chapter are consistent with those of experiment in the position and intensity of the absorption. Further, our results allow the identification of the admixed states via the polarization dependence of the absorption. The vibrations causing the admixture have a characteristic temperature dependence which allows their identification as well as for measurement of the magnitude of the coupling coeficient. 


\section{Eigenstates}

We now discuss the vibrational modes of anthracene. Then, allowing an interaction between the vibrations and the electrons, we shall perturb the electronic states of section 2, Chapter III.

There are 24 atoms in anthracene, therefore there are 72 normal modes. Of these, three are rotations of the rigid molecule and three translations. The remaining modes can be classified into symmetry types

$$
\begin{aligned}
12 a_{1 g} & +11 b_{1 g}+4 b_{2 g}+6 b_{3 g} \\
& +5 a_{1 u}+6 b_{1 u}+11 b_{2 u}+11 b_{3 u},
\end{aligned}
$$

the frequencies of these intramolecular vibrations have been calculated by Krainov, 40 (see Table 5-1 for reference).

The vibrations interact with the electrons via a potential $v_{e-v}$ thus perturbing the wave functions of the previous section. ${ }^{4}$ Take the electronic problem

$$
H_{0}|p\rangle=E_{p}|p\rangle
$$

and perturb it by $V_{e-v}$

$$
H=H_{0}+V_{e-v}
$$

then,

$$
H|R\rangle=\epsilon_{R}|R\rangle
$$

and in first order of perturbation theory:

$$
|R\rangle=|p\rangle+\sum_{q} \frac{|q\rangle\left\langle q\left|V_{e-v}\right| p\right\rangle}{E_{p}-E_{q}}
$$


TABIE 5-1

Normal modes of $\mathrm{C}_{1} \mathrm{H}_{10}$ (selected ones)

Symmetry $\hbar \omega(\text { calc. })^{40}$ coth $\frac{\hbar \omega}{2 k T}$ Symmetry $\hbar \omega(\text { calc. })^{40} \operatorname{coth} \frac{\hbar \omega}{2 k T}$

$\left(\mathrm{cm}^{-1}\right) \quad \mathrm{T}=473^{\circ} \mathrm{K} \quad\left(\mathrm{cm}^{-1}\right) \quad \mathrm{T}=473^{\circ} \mathrm{K}$

\begin{tabular}{|c|c|c|c|c|c|}
\hline \multirow{4}{*}{${ }^{a} \lg$} & 375 & 1.94 & \multirow{3}{*}{$b_{2} u$} & 210 & 3.23 \\
\hline & 623 & 1.35 & & 627 & 1.35 \\
\hline & 826 & 1.18 & & 922 & 1.13 \\
\hline & 1029 & 1.09 & & 1147 & 1.06 \\
\hline & 1127 & 1.06 & & 1246 & 1.05 \\
\hline & 1258 & 1.04 & & 1266 & 1.04 \\
\hline & 1396 & 1.03 & & 1450 & $1.0^{\prime}$ \\
\hline & 1499 & 1.02 & & 1623 & 1.0 \\
\hline & 1590 & 1.02 & & 3051 & 1.0 \\
\hline & 3040 & 1.00 & & 3062 & 1.0 \\
\hline & 3041 & 1.00 & & 3073 & 1.0 \\
\hline & 3062 & 1.00 & & & \\
\hline \multirow[t]{11}{*}{${ }^{b}$ lg } & 364 & 1.99 & $b_{3} u$ & 591 & 1.40 \\
\hline & 504 & 1.55 & & & \\
\hline & 894 & 1.14 & & 1011 & 1.1 \\
\hline & 1112 & 1.07 & & 1114 & 1.0 \\
\hline & 1202 & 1.05 & & 1181 & 1.0 \\
\hline & 1279 & 1.04 & & 1345 & 1.0 \\
\hline & 1396 & 1.03 & & 1387 & 1.0 \\
\hline & 1569 & 1.02 & & 1457 & 1.0 \\
\hline & 1633 & 1.01 & & $153^{4 / 4}$ & 1.0 \\
\hline & 3055 & 1.00 & & 3040 & 1.0 \\
\hline & 3068 & 1.00 & & & \\
\hline
\end{tabular}


We now expand the interaction in a Taylor series, in the normal coordinates of the molecule in the ground state, about the equilibrium position while keeping only the term linear in the normal mode coordinates:

$$
V_{e-v}=\left.\sum_{\alpha} \frac{\partial V_{e-y}}{\partial Q_{\alpha}}\right|_{Q_{\alpha}=0} Q_{\alpha}
$$

Therefore, with an obvious definition, using (5-4):

$$
|R\rangle=|p\rangle+\sum_{q, \alpha} \gamma_{q_{\alpha}} Q_{\alpha}|q\rangle
$$

For an example of a calculation of $\gamma$ see conway et al. 42

To complete the eigenstates we now introduce the vibrations themselves, and use the Born-Oppenheimer separation, ${ }^{43}$ Eiving

$$
\left.|R, \beta\rangle=|R>| \beta_{R}\right\rangle
$$

where $\beta_{R}$ represents a set of vibrational quantum numbers.

The importance of allowing interaction of the electrons and the vibrations is that optical transitions which at first sight appear forbidden by symmetry become allowed by the admixture of states due to the interaction. $41,42,44,14$

\section{Two-photon Absorption}

Odd parity vibrations can mix opposite parity states and therefore two-photon absorption may be observed at energies near predicted odd parity states. The absorption will have the character, (angular dependence and symmetry characteristics), of the even parity 
state mixed in. Even in the case of several even parity states mixed in appreciably, each even parity state will give rise to a separate absorption series. In general, each absorption series will start, (in energy), at approximately the energy of the first peak in one-photon transitions plus one quantum of the vibration mixing in that state. The states mixed in may be identified by the predictions of Chapter 3, if they are resolved. The necessary change to the results of Chapter 3 is a reduction in the magnitude of the absolute absorption by the square of the coupling coeficient. This correction has a distinctive temperature dependence coth $\frac{\hbar \omega}{2 k T}$ which, if observable, will identify the vibration causing the mixing and the strength of the coupling.

For the wave functions, we refer to equations (5-6) and (5-7), and apply to anthracene. For an excited state we have

$$
|R, \beta\rangle=\left(|p\rangle+\sum_{q, \alpha} \gamma_{q \alpha} Q_{\alpha}\left|q_{p}\right\rangle\right)\left|\beta_{p}\right\rangle
$$

We shall assume that the perturbation of the ground state via the electron-vibrational interaction is negligible due to the large energy separations which appear in the denominator of $\gamma$. Thus, the ground state is given by

$$
\left|A_{1 g}, \beta\right\rangle=\left|A_{1 g}\right\rangle\left|\beta_{0}\right\rangle
$$

Two-photon transitions can be computed via (3-11). The vibrational effects appear only in the second order matrix element and the line shape now appears explicitly rather than phenomenologically. 
$\left|R^{(2)}\right|_{g}^{2}(2 \hbar \omega)=\left|\sum_{i, j} \sum_{m, \beta_{m}} \frac{\left\langle R \beta_{R}\left|x_{i}\right| m \beta_{m}\right\rangle\left\langle m \beta_{m}\left|x_{j}\right| A_{\mid g} \beta_{0}\right\rangle}{E_{m} \beta_{m}-\hbar \omega}\right|^{2}$

Here we include the appropriate direction cosine in the coordinate. Note that the energy in the denominator includes the vibrational energy and that the intermediate states include vibrational wave functions as products. Using equations ( $5-8 a$ and b), (5-9) becomes

$$
\left|\sum_{i, j} \sum_{m, \beta_{m}} \frac{\left\langle p \beta_{p}\left|x_{i}\right| m \beta_{m}\right\rangle\left\langle m \beta_{m}\left|x_{j}\right| A_{1 g} \beta_{0}\right\rangle+\sum_{q, \alpha} \gamma_{q \alpha}\left\langle q \beta_{p}\left|Q_{\alpha} x_{i}\right| m \beta_{m}\left\langle m \beta_{m}\left|x_{j}\right| A_{1 g} \beta_{0}\right\rangle\right.}{E_{m \beta_{m}}-\hbar \omega}\right|^{2}
$$

The case of interest is that in which the first term vanishes due to the parity selection rule. In this case $|p\rangle$ is an odd parity state. We wish to account for the observed two-photon absorption ${ }^{16}$ in anthracene (at $2 \hbar \omega=3.57 \mathrm{eV})$.

Pariser ${ }^{27}$ predicts two low lying odd parity states in the appropriate energy region, a $\mathrm{B}_{2 \mathrm{u}}^{+}$state at $3.65 \mathrm{eV}$ and a $\mathrm{B}_{3 \mathrm{u}}^{-}$state at $3.71 \mathrm{eV}$. The former is well known experimentally from one-photon spectroscopy to be at $3.28 \mathrm{eV}$, the latter is not observable since it has vanishing dipole matrix element with the ground state (only - - + transitions have non-vanishing dipole m.e. (see 27)). Nevertheless, the - state is available as a parent state for mixing in the even parity (-) states via the vibrations. Since we are concerned with mixing an even parity, - , state with an odd parity state via the electron-vibrational interaction, it would seem, merely on symmetry grounds, that the most suited odd parity state would be + since it 
will have non-vanishing dipole matrix element. To attempt to mix an odd parity, - state would give a smaller coupling since we would have to go beyond the dipole terms. The odd parity state being perturbed determines the energy of the absorption and the symmetry of the vibrations causing the mixing.

We explain the observed two-photon absorption of molecular anthracene with the model having one or both of the $(4.9 \mathrm{eV}) \mathrm{B}_{\mathrm{Ig}}$ and $(5.0 \mathrm{eV}) \mathrm{A}_{\mathrm{lg}}^{*}$ states mixed, by vibrations, into the $\mathrm{B}_{2 \mathrm{u}}$ state. Note that the line shape function is already contained in the square of the second order matrix element since conservation of energy, at $2 \hbar \omega$, will determine the final vibrational state $\left|\beta_{p}\right\rangle$ and the vibrational matrix elements determine the line shape. The only nonvanishing $\gamma_{\mathrm{A}_{I g}} \alpha^{\prime}$ are those where the symmetry of the vibration, $\left(\alpha^{\prime}\right)$, is $b_{2 u}$ since otherwise the matrix element in the numerator of $\gamma_{\mathrm{A}_{\text {Ig }} \alpha} \alpha^{\prime}$ vanishes. Similarly, $\gamma^{\prime} \mathrm{B}_{\mathrm{Ig}} \alpha$ vanishes unless $\alpha$ is of

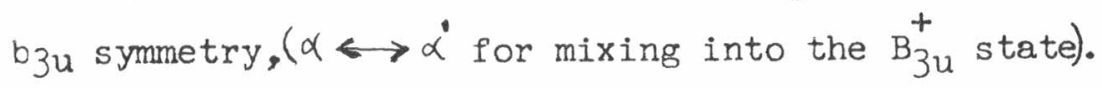

We now write the vibrational wave functions in the occupation representation. However, since the normal modes of the excited state are in general different from those of the ground state, (e.g., they certainly are different if there is a Stokes shift), we expand each of the representations of the excited state in a linear combination of a complete set in the ground state, one of the same symmetry of course.

$$
\left\langle\beta_{p}\right|=\prod_{n_{p}}\left\langle\beta_{n_{p}}\right|=\prod_{n_{p}} \sum_{\beta_{n_{0}}^{\prime}}\left\langle\beta_{n_{p}} \mid \beta_{n_{0}}^{\prime}\right\rangle\left\langle\beta_{n_{0}}^{\prime}\right|
$$


and $\left\langle\beta_{0}\left|=\frac{\pi}{n_{0}}<\beta_{n_{0}}\right|\right.$

Continuing, the matrix elements need in (5-10) are 1,14

$$
\left\langle\beta_{p} \mid \beta_{0}\right\rangle=\prod_{n_{p}, n_{0} \neq \alpha}\left\langle\beta_{n_{p}} \mid \beta_{n_{0}}\right\rangle \delta_{\beta_{\alpha_{p}}, \beta_{\alpha}}
$$

and

$$
\begin{aligned}
\left\langle\beta_{p}\left|Q_{\alpha}\right| \beta_{0}\right\rangle=\prod_{n_{p} n_{0} \neq \alpha} & \left\langle\beta_{n_{p}} \mid \beta_{n_{0}}\right\rangle \\
x & <\beta_{\alpha}+1\left|Q_{\alpha}\right| \beta_{\alpha}>\delta_{\beta_{\alpha}, \beta_{\alpha}+1}
\end{aligned}
$$

We have assumed, at least approximately, that for the normal mode involved in the mixing, $(\alpha)$, the mode in the excited state, $\left(\alpha_{p}\right)$, is the same as that of the ground state. That is,

$$
\left\langle\beta_{\alpha_{p}} \mid \beta_{\alpha}^{\prime}\right\rangle=\delta_{\beta_{\alpha_{p}}, \beta_{\alpha}^{\prime}}
$$

We further assume that $\mathrm{kT}<\hbar_{\alpha} \omega_{\text {so }}$ that the ground state is primarily in the lowest $\alpha$ vibrational state. It should be noted that for these temperatures the $B_{1 g}$ and $A_{1 g}$ absorptions do not occur at the same energies due to the one phonon selection rule implicit in $(5-12)$.

Using the results of equation $(5-12)$ in $(5-10)$, we obtain the influence of vibrations and the polarization dependence of the absorption:

$$
\begin{aligned}
w \propto A_{\text {orientations }} A_{\beta_{0}} \mid\left(l_{1} m_{2}+m_{1} l_{2}\right) \sum_{\alpha} \gamma_{B_{1 g} \alpha} \prod_{n_{p}, n_{0}}^{\prime}\left\langle\beta_{n_{p}} \mid \beta_{n_{0}}\right\rangle \\
\quad x\left[\delta_{\beta_{\alpha_{p}}, \beta_{\alpha}+1}\left\langle\beta_{\alpha}+1\left|Q_{\alpha}\right| \beta_{\alpha}\right\rangle\right] \frac{\left\langle B_{1 g}|x y| A_{1 g}\right\rangle}{E_{x y}-\hbar \omega}+
\end{aligned}
$$




$$
\begin{aligned}
& +\frac{\left\langle A_{1 g}^{*}\left|x^{2}\right| A_{1 g}\right\rangle}{\bar{E}_{x x}-\hbar \omega}\left(l_{1} \ell_{2}+\lambda B m_{1} m_{2}\right) \sum_{\alpha^{\prime}} \gamma_{A_{1 g}^{*} \alpha^{\prime}} \prod_{n_{p}, n_{0}}^{\prime}\left\langle\beta_{n_{p}} \mid \beta_{n_{0}}\right\rangle \\
& \left.\quad x \delta_{\beta_{\alpha_{p}^{\prime}}, \beta_{\alpha^{\prime}}+1}\left\langle\beta_{\alpha^{\prime}}+1\left|Q_{\alpha^{\prime}}\right| \beta_{\alpha^{\prime}}\right\rangle\right|^{2}
\end{aligned}
$$

where $\alpha$ runs over $b_{3 u}$ vibrations and $\alpha^{\prime}$ over $b_{2 u}$ vibrations. Each significant $\gamma$ will give rise to an absorption series beginning at the energy of the one-photon absorption $(3.3 \mathrm{eV})$ plus $\hbar \omega_{\alpha}$ and followed at higher energies by the usual phonon structure which is decreased in intensity by the square of the Frank-Condon integrals. The series will have the symmetry and angular dependence characteristics detailed in Chapter 3 corresponding to whether it arises from the admixing of the $\mathrm{A}_{\mathrm{lg}}^{*}(5.0 \mathrm{eV})$ or the $\mathrm{B}_{\mathrm{lg}}(4.9 \mathrm{eV})$ state.

To estimate the absolute absorption we assume $\left|\gamma_{Q}\right|^{2} \sim .01$ and keep all the parameters as given in equation (3-17). The results are:

1. from (3-17), for absorption due to the admixture of the $B_{l g}$ state:

$$
w^{B}=5.6 \times 10^{-50} F_{1} F_{2} A v\left|l_{1} m_{2}+m_{1} l_{2}\right|^{2} \quad \sec ^{-1}(5-15)
$$

at $2 \hbar \omega=3.3 \mathrm{ev}+\hbar \omega_{b_{3 u}}$, for a random sample. For values of the averages see equations $3-13,14,14$ a and b, Fig. 3-2. For ratios of the absorption due to different types of polarization see equations 3-15, 15a, and Fig. 3-2. For energies of the $b_{3 u}$ vibrations see Table 5-1.

2. from 3-24, for absorption due to the admixture of the 
$A_{\text {Ig }}^{*}(5.0 \mathrm{eV})$ state:

$$
W^{A}=2.6 \times 10^{-52} F_{1} F_{2} A v\left|\lambda_{1} l_{2}-0.844 \lambda m_{1} m_{2}\right|^{2} \sec ^{-1} \cdot(5-16)
$$

at $2 \hbar \omega=3.3 \mathrm{ev}+\hbar \omega_{b_{2} u}$, for a random sample. For values of the averages see equations 3-21, 22, 22a and b, and Fig. 3-3. For ratios of the absorption due to different types of polarization see Fig. 3-3, and Table 3-1. For energies of the $b_{2 u}$ vibrations see Table 5-1.

Finally, we wish to point out that for the integrated absorption we must do a sum over final states as well as the average over initial states. This sum over the band will give

$$
\begin{aligned}
& W=W_{A_{i g}^{*} A_{i g}} \sum_{\alpha}\left|\gamma_{A_{i g}^{*} \alpha}\right|^{2} \frac{\hbar}{2 M_{\alpha} \omega_{\alpha}} \operatorname{coth} \frac{\hbar \omega_{\alpha}}{2 k T}
\end{aligned}
$$

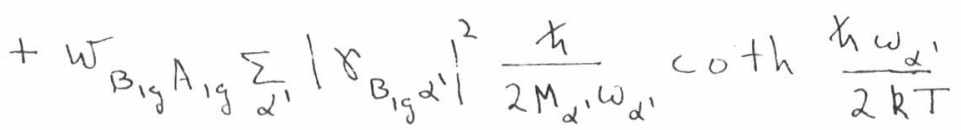

where $\alpha$ runs over $b_{2 u}$ vibrations, $\alpha^{\prime}$ over $b_{3 u}$ vibrations and if the temperature dependence is observable, the appropriate $\gamma^{\wedge}(\mathrm{s})$ and $\omega$ (s) may be identified, (see Table 5-1).

The only presently available experiments on molecular anthracene are those of Peticolas and Rieckhof ${ }^{18}$ on dilute solution of anthracene frozen in EPA glass. They measured the fluorescence, due to excitation with a ruby laser $(\hbar \omega=1.785 \mathrm{eV})$, which is equal to the transition rate multiplied by the quantum efficiency for fluorescent transitions $(\sim 1$, expected). Our predictions for the absolute transition rate for either $A_{1 g}^{*}$ of $B_{1 g}$ type final state are in order of magnitude agreement with their observation. The angular 
dependence ratios, etc. have not been measured though they should be best suited for identification of the states involved in the transition.

To summarize the energies at which two-photon absorption should be observable, we plot the vibronic states of molecular anthracene in Figure 5-1. In order to observe the absorption near $5 \mathrm{eV}$ an experiment might be possible in which two beams of different energies are incident on the sample, such as that of Frohlich and Mahr 55 in the solid. 


\section{Vibronic States of Molecule}

$$
\begin{aligned}
& B_{3 u} 5.24 \mathrm{eV} \\
& A_{1 g}^{*} 5.00 \mathrm{eV}=:= \\
& \mathrm{B}_{\text {Ig }} 4.94 \mathrm{eV}
\end{aligned}
$$

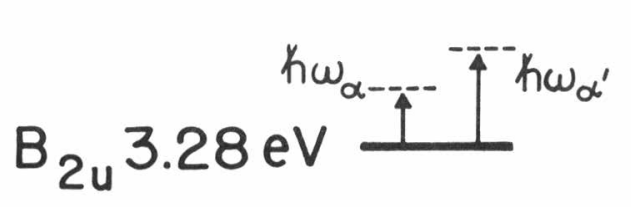

Both should absorb two photons directly
$A_{1 g}^{*}$ admixture due to $b_{2 u}$ vibr.

$B_{1 g}$ admixture due to $b_{3 u}$ vibr.

cause two photon absorption

Absorption observed at $3.57 \mathrm{eV}$

$$
A_{1 g} O \text { eV }
$$

Fig. 5-I 
CHAPTER VI

CONCLUSION

We have considered the absorption of two photons by anthracene. The absorption of the molecule, which has been observed, has been explained by suggesting that the final state is vibronic in character. The identification of the symmetries of the admixed states has been treated as well as the possibility of identifying the vibrations causing the mixing and the strength of the coupling. The energy shifts of even parity electronic states have been calculated in lowest order, and higher order interactions have been estimated. This calculation suggests that, in contrast with the molecular absorption, the absorption by the solid takes place directly to an even parity state. The angular dependence of the solid absorption has been calculated in order to permit the identification of the absorbing states.

In molecular samples, the calculation of the polarization dependence of absorption due to $\mathrm{B}_{\mathrm{lg}}$ states is dependent only on symmetry, thus the calculation is exact. For Alg final states the results have been parametrized in the hope that the correct parameter may be chosen by comparing theory and experiment. The absolute intensity of the absorption calculated is only approximate due to having chosen an average energy. It is of the correct order of magnitude. 
The calculation of the energy shifts in the solid has been treated approximately. The quadrupole-quadrupole contribution in first order of perturbation theory was calculated neglecting differential overlap which is expected to give a small contribution (10\%). However, higher order multipoles were not treated. The contribution to the energy shift of the Van der Waals interaction was estimated and found to be important. However, second order contributions to the Davydov splittings and the energy dispersion were estimated to have small importance for even parity exciton states.

The angular dependence of two-photon absorption in the solid was calculated taking into consideration the theory of crystal optics. This calculation is only approximate since the index anisotropy must be included in calculating the local fields. The Lorenz-Lorentz expressions used for the local fields are only of approximate validity.

Two-photon transitions should be observable with present experimental techniques to a $\mathrm{B} I g$ state at $4.94 \mathrm{eV}$ and to an $\mathrm{Alg}$ state at $5.00 \mathrm{eV}$ in the molecule; and to an $\mathrm{A}_{1 g}$-derived Davydov doublet near $4.8 \mathrm{eV}$ in the crystal. The energies of all these states may be in the neighborhood of $0.3 \mathrm{eV}$ lower than predicted due to the limitations of the wave functions.

The spectroscopic identification of the final states in twophoton absorption in anthracene has been the primary purpose of the work and the experiments suggested should accomplish that purpose. The material is such that the theories of two-photon absorption, symmetry, excitons, crystal optics and vibronic mixing have vital 
interplay.

Based on available experimental information we make the tentative assignments of the observed absorption to a $\mathrm{B}_{\mathrm{g}}$ state at $3.58 \mathrm{eV}$ and an $\mathrm{A}_{\mathrm{g}}$ state at $3.48 \mathrm{eV}$ in the crystal. These exciton states are derived from the $B_{l_{g}}$ electronic state of the molecule at $4.94 \mathrm{eV}$. In the molecule the assignment of the absorption is to a vibronic mixture of the lowest energy $B_{2} u$ electronic state with either or both of the Blg state at $4.94 \mathrm{eV}$ and the Alg state at $5.0 \mathrm{eV}$. 
APPENDIX A

Wave functions

This appendix gives the expressions for $\pi$-electron molecular orbitals for the polyacenes. ${ }^{37,38}$ Pariser's ${ }^{27}$ results for anthracene will be listed for reference.

$X_{\mu}$ is the $2 p \pi$ carbon function centered at atom $\mu$, (see Fig. 3-1 and $\mathrm{A}-1$ ), and

$$
\sigma_{\mu}^{ \pm}=x_{\mu} \pm x_{\mu}
$$

then, for

$$
H=\sum_{\mu}\left(H_{\text {core }}\right)_{\mu}+\sum_{p>q} \frac{e^{2}}{r_{p q}}
$$

where $\mathrm{p}$ and $\mathrm{q}$ run over $\pi$-electrons, only nearest neighbor interactions considered.

Hence

$$
\begin{aligned}
& \phi_{\lambda}^{ \pm}=\sum_{\mu} c_{\lambda \mu}^{ \pm} \sigma_{\mu}^{ \pm} \\
& H \phi_{\lambda}^{ \pm}=\left(E_{0}+k_{\lambda}^{ \pm} \beta\right) \phi_{\lambda}^{ \pm}
\end{aligned}
$$

then, for a molecule of $\mathrm{n}$ benzene rings: $:^{37}$

$$
\begin{array}{ll}
k_{\lambda}^{ \pm}= \pm \frac{1}{2}+\frac{1}{2} \cos \frac{\lambda \pi}{2(n+1)}\left(16+\sec ^{2} \frac{\lambda \pi}{2(n+1)}\right)^{1 / 2} & \\
\lambda \neq n+1 ; & k_{n+1}^{ \pm}= \pm 1.0
\end{array}
$$


(see Table A-1, for the $k \lambda$ in anthracene) and 38

$$
\begin{aligned}
& C_{\mu \lambda}^{ \pm}=\left[\frac{k_{\lambda}^{ \pm} \mp 1}{\left(2 k_{\lambda}^{ \pm} \mp 1\right)(n+1)}\right]^{1 / 2} \sin \frac{\mu \lambda \pi}{2(n+1)} \\
& \quad x\left[\cos ^{2} \frac{\mu \pi}{2}+\left(\frac{k_{\lambda}^{ \pm}}{k_{\lambda}^{ \pm} \mp 1}\right)^{1 / 2} \sin ^{2} \frac{\mu \pi}{2}\right] \quad(\lambda \neq n+1) \\
& C_{\mu, n+1}=\left(\frac{1}{2(n+1)}\right)^{1 / 2} \sin \frac{\mu \pi}{2}
\end{aligned}
$$

The values of these coefficients are listed in Table A-I for anthracene. The symmetry classes of the MO's are:

$$
\begin{aligned}
& \phi^{+}\left\{\begin{array}{l}
\lambda \text { odd : } b_{1 u} \\
\lambda \text { even: } b_{2 g}
\end{array}\right. \\
& \phi^{-} \begin{cases}\lambda \text { odd: } & b_{3 g} \\
\lambda \text { even: } & a_{1 u}\end{cases}
\end{aligned}
$$

The lowest energy configuration has the $2 n+1$ MO's characterized by positive $k_{\lambda}$ values filled with the $4 n+2 \pi$-electrons.

The wave functions are now constructed. First, configurations are built by antisymmetrizing the occupied Mo's: 


$$
\begin{aligned}
V_{0} & =(N !)^{-\frac{1}{2}} \sum_{p}(-1)^{p} \rho \phi_{1}(1) \alpha \phi_{1}(2) \beta \phi_{2}(3) \alpha \phi_{2}(4) \beta \ldots . \\
& =(1 T, 2 \overline{2}, \ldots . . .)
\end{aligned}
$$

$P$ is the usual permutation operator.

$$
V_{i k^{\prime}}=2^{-\frac{1}{2}}\left[\left(1, \ldots . i \overline{k^{\prime}}, \ldots\right)-\left(1, \ldots \bar{i} k^{\prime}, \ldots\right)\right]
$$

where $V_{i k}$ ' is an excited singlet configuration, and then allowing configuration interaction to construct the excited states:

$$
\Psi_{a}=A_{1 a} V_{1}+A_{2 a} V_{2}+\cdots .
$$

Table A-2 lists the configuration interaction coefficients for the even parity states of the anthracene molecule as calculated by Pariser. 27 


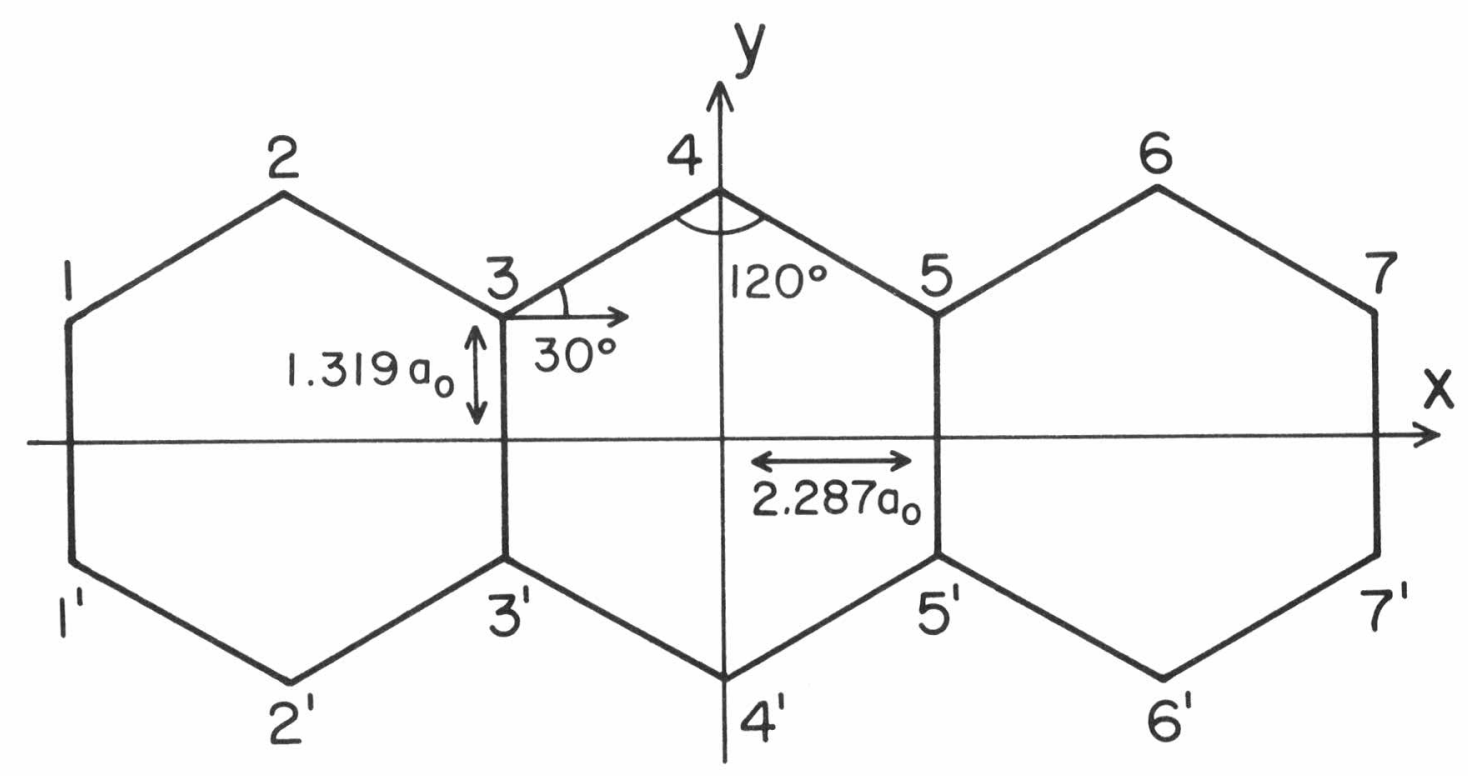

Fig. $A^{-1}$ 
TABLE $A-1$

Anthracene

\begin{tabular}{lrrrrrrrr}
$\lambda \mu$ & 1 & 2 & 3 & 4 & 5 & 6 & 7 & $\mathrm{k} \lambda$ \\
\hline $1^{+}$ & .15194 & .21488 & .36683 & .30389 & .36683 & .21488 & .15194 & 2.4142 \\
$2^{+}$ & .28867 & .28867 & .28867 & 0 & -.28867 & -.28867 & -.28867 & 2.0000 \\
$1^{-}$ & .11629 & .28076 & .28076 & .39705 & .28076 & .28076 & .11629 & 1.4142 \\
$3^{+}$ & .40626 & .16828 & -.16828 & -.23798 & -.16828 & .16828 & .40626 & 1.4142 \\
$2^{-}$ & .20412 & .40825 & .20412 & 0 & -.20412 & -.40825 & -.20412 & 1.0000 \\
$4^{+}$ & .35355 & 0 & -.35355 & 0 & .35355 & 0 & -.35355 & 1.0000 \\
$3^{-}$ & .21987 & .31094 & -.09107 & -.43973 & -.09107 & .31094 & .21987 & 0.4142 \\
$7^{-}$ & .15194 & -.21488 & .36683 & -.30389 & .36683 & -.21488 & .15194 & -2.4142 \\
$6^{-}$ & .28867 & -.28867 & .28867 & 0 & -.28867 & .28867 & -.28867 & -2.0000 \\
$7^{+}$ & .11629 & -.28076 & .28076 & -.39705 & .28076 & -.28076 & .11629 & -1.4142 \\
$5^{-}$ & .40626 & -.15828 & -.16828 & .23798 & -.16828 & -.16828 & .40626 & -1.4142 \\
$6^{+}$ & .20412 & -.40825 & .20412 & 0 & -.20412 & .40825 & -.20412 & -1.0000 \\
$4^{-}$ & .35355 & 0 & -.35355 & 0 & .35355 & 0 & -.35355 & -1.0000 \\
$5^{+}$ & .21987 & -.31094 & -.09107 & .43973 & -.09107 & -.31094 & .21987 & -0.4142
\end{tabular}





\section{APPENDIX B}

\section{Angular Averages}

We now detail the angular averages which represent the absorption of two photons by a random sample. It is completely equivalent to consider light incident on molecules which have random orientations with respect to the incidence or light which is Incident randomly on an oriented molecule. We shall use the latter approach.

Consider the molecular coordinate system $\mathrm{x}, \mathrm{y}, \mathrm{z}$ and perform the three Euler rotations, following Goldstein. 52 That is, a rotation by $\phi$ counter-clockwise about the $z$ axis yields a new coordinate system $x_{1}, y_{1}, z_{1}$; the new coordinate system is rotated by $\theta$ about the $x_{1}$ axis giving the system $x_{2}, y_{2}, z_{2}$; finally a rotation by $\psi$ about the $z_{2}$ axis yields a completely general coordinate system $x^{\prime}$, $y^{\prime}$ and $z^{\prime}$.

We begin with two polarization vectors of the photons to be absorbed $\hat{\epsilon}_{1,2}$ and define

$$
\hat{\epsilon}_{i}=l_{i} \hat{x}_{m}+m_{i} \hat{y}_{m}+n_{i} \hat{z}_{m}
$$

if the light incident is unpolarized the two photons can have independently arbitrary polarization. However, if we restrict ourselves to one beam, the light is polarized, both of the photons that can be absorbed from a polarized beam will have the same polarization. 
For absorption to a state of $\mathrm{B}_{1 g}$ symmetry, we need to average the expression:

$$
W_{B}=P A v\left|l_{1} m_{2}+m_{1} l_{2}\right|^{2}
$$

where $P$ is a polarization-independent constant. On the other hand, for transitions to a state of $\mathrm{A}_{1 g}$ symmetry we need to average

$$
W_{A}=Q A V\left|l_{1} l_{2}+C m_{1} m_{2}\right|^{2}
$$

with $Q$ also polarization independent.

Unpolarized light:

We choose the polarization vectors along $z^{\prime}$ axes in rotated and unrelated coordinate systems. We now use the rotation matrix to relate the polarization to the direction cosines in the system of the molecule:

$$
\left(\begin{array}{l}
\theta_{i} \\
m_{i} \\
n_{i}
\end{array}\right)=\left(\begin{array}{c}
\sin \theta_{i} \sin \phi_{i} \\
-\sin \theta_{i} \cos \phi_{i} \\
\cos \theta_{i}
\end{array}\right)
$$

Therefore,

$$
\begin{aligned}
w_{B}=P A_{V} A_{v} & \mid \sin \theta_{1} \sin \phi_{1} \sin \theta_{2} \cos \phi_{2} \\
& +\left.\sin \theta_{2} \sin \phi_{2} \sin \theta_{1} \cos \phi_{1}\right|^{2}
\end{aligned}
$$

and

$$
\begin{aligned}
W_{A}=Q \operatorname{Av}_{\Omega_{1} \Omega_{2}} A_{V} \mid \sin \theta_{1} \sin \phi_{1} \sin \theta_{2} \sin \phi_{2} \\
+\left.C_{-} \sin \theta_{1} \cos \phi_{1} \sin \theta_{2} \cos \phi_{2}\right|^{2}
\end{aligned}
$$


The averages over solid angle $\Omega$ are defined

$$
A_{\Omega} v(\theta, \phi, \psi)=\frac{1}{4 \pi} \int_{0}^{2 \pi} d \psi \int_{0}^{2 \pi} d \phi \int_{0}^{\pi} \cos \theta d \theta \quad f(\theta, \phi, \psi)
$$

The result of using $(B-6)$ in $(B-5, a, b)$ is

$$
w_{B}=\frac{p}{q}
$$

and

$$
W_{A}=\frac{Q}{q}\left(1+c^{2}\right)
$$

Polarized light:

We are now restricted to $\hat{\epsilon}_{1}=\hat{\epsilon}_{2}$ and we treat the case of elliptically polarized light as a general case which includes linear and circular polarized light as its extremes. We choose a rotated coordinate system such that the direction of propagation is along $z^{\prime}$, then we choose an angle $\psi$ such that the light is described by

$$
\hat{\epsilon}=\frac{\hat{x}^{\prime}+i a \hat{y}^{\prime}}{\left(1+a^{2}\right)^{1 / 2}}
$$

where $a$ is a real number which we call the elliplicity. Once again the rotation matrix is used to describe the direction cosines in the coordinate system of the molecule. This procedure yields

$$
\left(\begin{array}{l}
l \\
m \\
n
\end{array}\right)=(1+a)^{-\frac{1}{2}}\left(\begin{array}{c}
\cos \psi \cos \phi-\cos \theta \sin \phi \sin \psi-i a(\sin \psi \cos \phi+\cos \theta \sin \phi \cos \psi) \\
\sin \phi+\cos \theta \cos \phi \sin \psi-i a(\sin \psi \sin \phi-\cos \theta \cos \phi \cos \psi) \\
\sin \theta \sin \psi+i a \sin \theta \cos \psi
\end{array}\right)
$$


Using ( $B-9$ ) in (B-2) and ( $B-3$ ), while remembering that $\hat{\epsilon}_{1}=\hat{\epsilon}_{2}$, yields the expressions to be averaged by means of (B-6). The result of this averaging procedure is

$$
w_{B}=\frac{4 P}{\left(1+a^{2}\right)^{2}} \quad \frac{a^{4}+4 a^{2}+1}{15}
$$

and $W_{A}=\frac{Q}{15\left(1+a^{2}\right)^{2}}\left[\left(3 a^{4}+2 a^{2}+3\right)\left(1+c^{2}\right)\right.$

$$
\left.+\frac{c}{8}\left(21 a^{4}-101 a^{2}+16\right)\right]
$$

which may be specializing to linearly polarized light $a=0$ or circularly polarized light $\mathrm{a}=1$.

The results of this appendix are used in Chapter 3. 
APPENDIX C

Matrix Elements

We shall detail the calculation of the matrix elements needed for the energy shift calculation of Chapter IV and for the transition rates calculations of Chapter III. The former calculation involves matrix elements in the interaction coordinate system but these can be transformed into matrix elements in the molecular coordinate system which are also the ones needed for the transition rates calculations. The general type of matrix element needed is

$$
\left.M_{E_{1} E_{2}}(\alpha \beta)=\int \Phi_{E_{1}}(\underline{r}) r_{\alpha} r_{\beta} \Phi_{E_{2}}(r)\right) d \underset{\sim}{r}
$$

$\mathrm{E}_{1,2}$ are the set of quantum numbers identifyjing the states of the free molecule, $r_{\alpha}$ and $r_{\beta}$ stand for $x, y$ or $z$ in the molecular basis set. Along with $\underset{\sim}{r}, r_{\alpha}$ and $r_{\beta}$ are 14 -electron coordinates. Thus, in one electron language

$$
M_{E_{1} E_{2}}(\alpha \beta)=\sum_{i, j=1}^{14} \int \Phi_{E_{1}}\left(\underline{r}_{1} \cdots r_{-14}\right) r_{\alpha i} r_{\beta j} \Phi_{E_{2}}\left(r_{i} \cdots \underline{r}_{14}\right) \prod_{m=1}^{14} d r_{m}
$$

and we must deal with matrix elements with $i=j$, one electron integrals, and $i \neq j$, two electron integrals. Since $\bar{\Phi}_{E}\left(r_{1} \ldots r_{14}\right)$ are linear combinations of Slater determinants of one electron functions, the rules for reducing the problem to the evaluation of one electron, one center integrals are well known and present only difficulties in bookkeeping. 
The results are tabulated in Chapter III.

The wave functions are those of Appendix A. The matrix elements are calculated through direct application of equations 9-13 in reference 27 by identifying formally our one- and two-electron operators with his although the operators themselves are different. 57 Finally, all integrals reduce to two types. First, there are linear combinations of the coordinates of the atoms. We used regular hexagons for anthracene with the interatomic distance taken as the average of the measurements in reference 39. Then there are integrals of the type

$$
\int x^{2}|X(r)|^{2} d r
$$

which are matrix elements involving the coordinates of the $2 p \pi$ carbon electron about its own nuclei. For these we used the Slater functions suggested by Pariser. 
TABLE D-I

APPENDIX D

$$
\underline{R}=a \underline{a}+b \underline{b}+c \underline{c}
$$

( $R$ in units of $10 a_{0}$ )

\begin{tabular}{|c|c|c|c|c|c|c|c|}
\hline a & $\mathrm{b}$ & c & $\mathrm{R}$ & $a$ & $\mathrm{~b}$ & c & $\mathrm{R}$ \\
\hline .50 & 0.50 & 0.00 & 0.9900 & 1.50 & -0.50 & 0.00 & 2.5000 \\
\hline .50 & -0.50 & 0.00 & 0.9900 & -1.50 & 0.50 & 0.00 & 2.5000 \\
\hline .50 & 0.50 & 0.00 & 0.9900 & -1.50 & -0.50 & 0.00 & 2.5000 \\
\hline .50 & -0.50 & 0.00 & 0.9900 & 2.00 & 0.00 & 1.00 & 2.6900 \\
\hline .00 & 1.00 & 0.00 & 1.1400 & -2.00 & 0.00 & -1.00 & 2.6900 \\
\hline .00 & -1.00 & 0.00 & 1.1400 & .50 & 0.50 & -1.00 & 2.7100 \\
\hline 1.00 & 0.00 & 0.00 & 1.6200 & .50 & -0.50 & -1.00 & 2.7100 \\
\hline-1.00 & 0.00 & 0.00 & 1.6200 & -.50 & 0.50 & 1.00 & 2.7100 \\
\hline 1.00 & 0.00 & 1.00 & 1.7900 & -.50 & -0.50 & 1.00 & 2.7100 \\
\hline-1.00 & 0.00 & -1.00 & 1.7900 & 1.50 & 1.50 & 1.00 & 2.7300 \\
\hline-.50 & 0.50 & -1.00 & 1.8600 & 1.50 & -1.50 & 1.00 & 2.7300 \\
\hline .50 & 0.50 & 1.00 & 1.8600 & -1.50 & 1.50 & -1.00 & 2.7300 \\
\hline .50 & -0.50 & -1.00 & 1.8600 & -1.50 & -1.50 & -1.00 & 2.7300 \\
\hline .50 & -0.50 & 1.00 & 1.8600 & 1.00 & 2.00 & 0.00 & 2.8000 \\
\hline .50 & 1.50 & 0.00 & 1.8900 & -.2 .00 & -2.00 & 0.00 & 2.8000 \\
\hline .50 & 1.50 & 0.00 & 1.8900 & 1.00 & -2.00 & 0.00 & 2.8000 \\
\hline .50 & -1.50 & 0.00 & 1.8900 & -1.00 & 2.00 & 0.00 & 2.8000 \\
\hline-.50 & -1.50 & 0.00 & 1.8900 & 1.00 & 2.00 & 1.00 & 2.9000 \\
\hline 1.00 & 1.00 & 0.00 & 1.9800 & -1.00 & -2.00 & -1.00 & 2.9000 \\
\hline-1.00 & -1.00 & 0.00 & 1.9800 & 1.00 & -2.00 & 1.00 & 2.9000 \\
\hline 1.00 & -1.00 & 0.00 & 1.9800 & -1.00 & 2.00 & -1.00 & 2.9000 \\
\hline-1.00 & 1.00 & 0.00 & 1.9800 & 2.00 & 1.00 & 1.00 & 2.9200 \\
\hline .00 & 0.00 & 1.00 & 2.1100 & -2.00 & -1.00 & -1.00 & 2.9200 \\
\hline .00 & 0.00 & -1.00 & 2.1100 & 2.00 & -1.00 & 1.00 & 2.9200 \\
\hline 1.00 & 1.00 & 1.00 & 2.1200 & -2.00 & 1.00 & -1.00 & 2.9200 \\
\hline-1.00 & -1.00 & -1.00 & 2.1200 & -.50 & 2.50 & 0.00 & 2.9600 \\
\hline 1.00 & -1.00 & 1.00 & 2.1200 & .50 & 2.50 & 0.00 & 2.9600 \\
\hline-1.00 & 1.00 & -1.00 & 2.1200 & -.50 & -2.50 & 0.00 & 2.9500 \\
\hline 1.50 & 0.50 & 1.00 & 2.2000 & .50 & -2.50 & 0.00 & 2.9600 \\
\hline 1.50 & -0.50 & 1.00 & 2.2000 & 1.50 & 1.50 & 0.00 & 2.9700 \\
\hline-1.50 & 0.50 & -1.00 & 2.2000 & 1.50 & -1.50 & 0.00 & 2.9700 \\
\hline-1.50 & -0.50 & -1.00 & 2.2000 & -1.50 & 1.50 & 0.00 & 2.9700 \\
\hline .00 & 2.00 & 0.00 & 2.2800 & -1.50 & -1.50 & 0.00 & 2.9700 \\
\hline .00 & -2.00 & 0.00 & 2.2800 & .00 & 2.00 & 1.00 & 3.1200 \\
\hline .00 & 1.00 & 1.00 & 2.4000 & .00 & -2.00 & -1.00 & 3.1200 \\
\hline .00 & -1.00 & -1.00 & 2.4000 & .00 & 2.00 & -1.00 & 3.1200 \\
\hline .00 & 1.00 & -1.00 & 2.4000 & .00 & -2.00 & 1.00 & 3.1200 \\
\hline .00 & -1.00 & 1.00 & 2.4000 & .50 & 1.50 & -1.00 & 3.1500 \\
\hline .50 & 1.50 & -1.00 & 2.4600 & -.50 & 1.50 & 1.00 & 3.1500 \\
\hline .50 & 1.50 & 1.00 & 2.4600 & .50 & -1.50 & -1.00 & 3.1500 \\
\hline .50 & -1.50 & -1.00 & 2.4600 & -.50 & -1.50 & 1.00 & 3.1500 \\
\hline .50 & -1.50 & 1.00 & 2.4600 & 2.00 & 0.00 & 0.00 & 3.2400 \\
\hline 1.50 & 0.50 & 0.00 & 2.5000 & -2.00 & 0.00 & 0.00 & 3.2400 \\
\hline
\end{tabular}


TABLE D-I (Cont.)

\begin{tabular}{|c|c|c|c|c|c|c|c|}
\hline$a$ & b & c & $R$ & a & $b$ & c & $\underline{R}$ \\
\hline 1.00 & 0.00 & -1.00 & 3.3100 & -.50 & 2.50 & 1.00 & 3.8900 \\
\hline-1.00 & 0.00 & 1.00 & 3.3100 & -.50 & -2.50 & 1.00 & 3.8900 \\
\hline-.50 & 2.50 & -1.00 & 3.3600 & -2.00 & -2.00 & 0.00 & 3.9600 \\
\hline .50 & 2.50 & 1.00 & 3.3600 & 2.00 & 2.00 & 0.00 & 3.9600 \\
\hline-.50 & -2.50 & -1.00 & 3.3600 & 2.00 & -2.00 & 0.00 & 3.9600 \\
\hline .50 & -2.50 & 1.00 & 3.3600 & -2.00 & 2.00 & 0.00 & 3.9600 \\
\hline 2.50 & 0.50 & 1.00 & 3.3800 & .00 & -3.00 & 1.00 & 4. 0200 \\
\hline 2.50 & -0.50 & 1.00 & 3.3800 & .00 & 3.00 & -1.00 & 4.0200 \\
\hline-2.50 & 0.50 & -1.00 & 3.3800 & .00 & 3.00 & 1.00 & 4.0200 \\
\hline-2.50 & -0.50 & -1.00 & 3.3800 & .00 & -3.00 & -1.00 & 4.0200 \\
\hline .00 & 3.00 & 0.00 & 3.4200 & 1.00 & -2.00 & -1.00 & 4.0200 \\
\hline .00 & -3.00 & 0.00 & 3.4200 & -1.00 & 2.00 & 1.00 & 4.0200 \\
\hline 2.00 & 1.00 & 0.00 & 3.4400 & 1.00 & 2.00 & -1.00 & 4.0200 \\
\hline-2.00 & -1.00 & 0.00 & 3.4400 & -1.00 & -2.00 & 1.00 & 4.0200 \\
\hline 2.00 & -1.00 & 0.00 & 3.4400 & 3.00 & 0.00 & 1.00 & 4.0500 \\
\hline-2.00 & 1.00 & 0.00 & 3.4400 & -3.00 & 0.00 & -1.00 & 4.0500 \\
\hline-1.00 & 1.00 & 1.00 & 3.5000 & 1.50 & 0.50 & -1.00 & 4.0600 \\
\hline 1.00 & -1.00 & -1.00 & 3.5000 & 1.50 & -0.50 & -1.00 & 4.0600 \\
\hline-1.00 & -1.00 & 1.00 & 3.5000 & -1.50 & 0.50 & 1.00 & 4.0600 \\
\hline 1.00 & 1.00 & -1.00 & 3.5000 & -1.50 & -0.50 & 1.00 & 4.0600 \\
\hline-2.00 & -2.00 & -1.00 & 3.5100 & .50 & 3.50 & 0.00 & 4.0700 \\
\hline 2.00 & 2.00 & 1.00 & 3.5100 & -.50 & 3.50 & 0.00 & 4.0700 \\
\hline 2.00 & -2.00 & 1.00 & 3.5100 & .50 & -3.50 & 0.00 & 4.0700 \\
\hline-2.00 & 2.00 & 1.00 & 3.5100 & -.50 & -3.50 & 0.00 & 4.0700 \\
\hline 1.50 & 2.50 & 1.00 & 3.5500 & 2.50 & 0.50 & 0.00 & 4.0900 \\
\hline-1.50 & 2.50 & -1.00 & 3.5500 & -2.50 & 0.50 & 0.00 & 4.0900 \\
\hline 1.50 & -2.50 & 1.00 & 3.5500 & 2.50 & -0.50 & 0.00 & 4.0900 \\
\hline-1.50 & -2.50 & -1.00 & 3.5500 & -2.50 & -0.50 & 0.00 & 4.0900 \\
\hline 1.50 & 2.50 & 0.00 & 3.7400 & 3.00 & 1.00 & 1.00 & 4.2100 \\
\hline 1.50 & -2.50 & 0.00 & 3.7400 & -3.00 & -1.00 & -1.00 & 4.2100 \\
\hline-1.50 & 2.50 & 0.00 & 3.7400 & 3.00 & -1.00 & 1.00 & 4.2100 \\
\hline-1.50 & -2.50 & 0.00 & 3.7400 & -3.00 & 1.00 & -1.00 & 4.2100 \\
\hline 2.50 & 1.50 & 1.00 & 3.7500 & -2.00 & 3.00 & -1.00 & 4.3400 \\
\hline 2.50 & -1.50 & 1.00 & 3.7500 & 2.00 & -3.00 & 1.00 & 4.3400 \\
\hline-2.50 & 1.50 & -1.00 & 3.7500 & 2.00 & 3.00 & 1.00 & 4.3400 \\
\hline-2.50 & -1.50 & -1.00 & 3.7500 & -2.00 & -3.00 & -1.00 & 4.3400 \\
\hline-1.00 & -3.00 & 0.00 & 3.7800 & 1.50 & 1.50 & -1.00 & 4.3700 \\
\hline 1.00 & 3.00 & 0.00 & 3.7800 & 1.50 & -1.50 & -1.00 & 4.3700 \\
\hline 1.00 & -3.00 & 0.00 & 3.7800 & -1.50 & 1.50 & 1.00 & 4.3700 \\
\hline-1.00 & 3.00 & 0.00 & 3.7800 & -1.50 & -1.50 & 1.00 & 4.3700 \\
\hline-1.00 & -3.00 & -1.00 & 3.8600 & .50 & 3.50 & 1.00 & 4.3700 \\
\hline 1.00 & 3.00 & 1.00 & 3.8600 & .50 & -3.50 & 1.00 & 4.3700 \\
\hline 1.00 & -3.00 & 1.00 & 3.8600 & -.50 & 3.50 & -1.00 & 4.3700 \\
\hline-1.00 & 3.00 & -1.00 & 3.8600 & -.50 & -3.50 & -1.00 & 4.3700 \\
\hline .50 & 2.50 & -1.00 & 3.8900 & 2.50 & 2.50 & 1.00 & 4.3900 \\
\hline .50 & -2.50 & -1.00 & 3.8900 & 2.50 & -2.50 & 1.00 & 4.3900 \\
\hline
\end{tabular}


TABLE D-I (Cont.)

\begin{tabular}{|c|c|c|c|c|c|c|c|}
\hline$a$ & b & c & R & a & $b$ & $c$ & $B$ \\
\hline-2.50 & 2.50 & -1.00 & 4.3900 & 1.00 & -4.00 & 1.00 & 4.8900 \\
\hline-2.50 & -2.50 & -1.00 & 4.3900 & -1.00 & 4.00 & -1.00 & 4.8900 \\
\hline 2.50 & 1.50 & 0.00 & 4.4000 & 2.00 & 1.00 & -1.00 & 4.9000 \\
\hline 2.50 & -1.50 & 0.00 & 4.4000 & -2.00 & -1.00 & 1.00 & 4.9000 \\
\hline-2.50 & 1.50 & 0.00 & 4.4000 & 2.00 & -1.00 & -1.00 & 4.9000 \\
\hline-2.50 & -1.50 & 0.00 & 4.4000 & -2.00 & 1.00 & 1.00 & 4.9000 \\
\hline 1.50 & 3.50 & 1.00 & 4.5200 & 1.50 & 2.50 & -1.00 & 4.9300 \\
\hline 1.50 & -3.50 & 1.00 & 4.5200 & 1.50 & -2.50 & -1.00 & 4.9300 \\
\hline-1.50 & 3.50 & -1.00 & 4.5200 & -1.50 & 2.50 & 1.00 & 4.9300 \\
\hline-1.50 & -3.50 & -1.00 & 4.5200 & -1.50 & -2.50 & 1.00 & 4.9300 \\
\hline .00 & 4.00 & 0.00 & 4.5600 & 2.50 & 2.50 & 0.00 & 4.9500 \\
\hline .00 & -4.00 & 0.00 & 4.5600 & 2.50 & -2.50 & 0.00 & 4.9500 \\
\hline 3.00 & 2.00 & 1.00 & 4.6500 & -2.50 & 2.50 & 0.00 & 4.9500 \\
\hline-3.00 & -2.00 & -1.00 & 4.6500 & -2.50 & -2.50 & 0.00 & 4.9500 \\
\hline 3.00 & -2.00 & 1.00 & 4.6500 & -3.00 & -1.00 & 0.00 & 4.9900 \\
\hline-3.00 & 2.00 & -1.00 & 4.6500 & 3.00 & 1.00 & 0.00 & 4.9900 \\
\hline 1.50 & 3.50 & 0.00 & 4.6700 & 3.00 & -1.00 & 0.00 & 4.9900 \\
\hline 1.50 & -3.50 & 0.00 & 4.6700 & -3.00 & 1.00 & 0.00 & 4.9900 \\
\hline-1.50 & 3.50 & 0.00 & 4.6700 & & & & \\
\hline-1.50 & -3.50 & 0.00 & 4.6700 & & & & \\
\hline-2.00 & -3.00 & 0.00 & 4.7100 & & & & \\
\hline 2.00 & 3.00 & 0.00 & 4.7100 & & & & \\
\hline 2.00 & -3.00 & 0.00 & 4.7100 & & & & \\
\hline-2.00 & 3.00 & 0.00 & 4.7100 & & & & \\
\hline 1.00 & 3.00 & -1.00 & 4.7600 & & & & \\
\hline-1.00 & -3.00 & 1.00 & 4.7600 & & & & \\
\hline 2.00 & 0.00 & -1.00 & 4.7600 & & & & \\
\hline-2.00 & 0.00 & .2 .00 & 4.7600 & & & & \\
\hline 1.00 & -3.00 & -1.00 & 4.7600 & & & & \\
\hline-1.00 & 3.00 & 1.00 & 4.7600 & & & & \\
\hline .50 & 3.50 & -1.00 & 4.7900 & & & & \\
\hline .50 & -3.50 & -1.00 & 4.7900 & & & & \\
\hline-.50 & 3.50 & 1.00 & 4.7900 & & & & \\
\hline-.50 & -3.50 & 1.00 & 4.7900 & & & & \\
\hline 3.50 & 0.50 & 1.00 & 4.8300 & & & & \\
\hline 3.50 & -0.50 & 1.00 & 4.8300 & & & & \\
\hline-3.50 & 0.50 & -1.00 & 4.8300 & & & & \\
\hline-3.50 & -0.50 & -1.00 & 4.8300 & & & & \\
\hline 1.00 & 4.00 & 0.00 & 4.8400 & & & & \\
\hline-1.00 & -4.00 & 0.00 & 4.8400 & & & & \\
\hline 1.00 & -4.00 & 0.00 & 4.8400 & & & & \\
\hline-1.00 & 4.00 & 0.00 & 4.8400 & & & & \\
\hline 3.00 & 0.00 & 0.00 & 4.8600 & & & & \\
\hline-3.00 & 0.00 & 0.00 & 4.8600 & & & & \\
\hline 1.00 & 4.00 & 1.00 & 4.8900 & & & & \\
\hline-1.00 & -4.00 & -1.00 & 4.8900 & & & & \\
\hline
\end{tabular}




\section{APPENDIX E}

Wave Vector Dependence of Energy

We tabulate the values of the energy of the exciton states as a function of wave vector. $J$ is the value of the wave vector in units of $\pi /|a \underline{a l} \pi /| \underset{b}{b}$ or $\pi /|\leq|$ respectively for the three sets of entries in each table. The energies are given in milli-ev. The second and third columns are the calculation of the $Q-Q$ interaction. The fourth and last columns are the Maximum Van der Waals interaction calculation.

For this appendix

$$
E_{i} \pm(\underline{R})=\Delta_{i}(\underline{k}) \pm d_{i}(\underline{k})
$$

with the zero of energy taken at $E_{i}-E_{O}+D_{i}$ as defined in the text.

$$
\mathrm{k}_{\mathrm{a}} \text { is along } \underline{\mathrm{b}} \times \underline{\mathrm{c}}, \mathrm{k}_{\mathrm{b}} \text { along } \underline{\mathrm{c}} \times \underline{\mathrm{a}} \text { and } \mathrm{k}_{\mathrm{c}} \text { along } \underline{\mathrm{a}} \mathrm{x} \underline{\mathrm{b}} \text {. }
$$


TABLE E-I

$k_{a}$

$4.9 \mathrm{eV}, \mathrm{B}_{\mathrm{Ig}}$ state

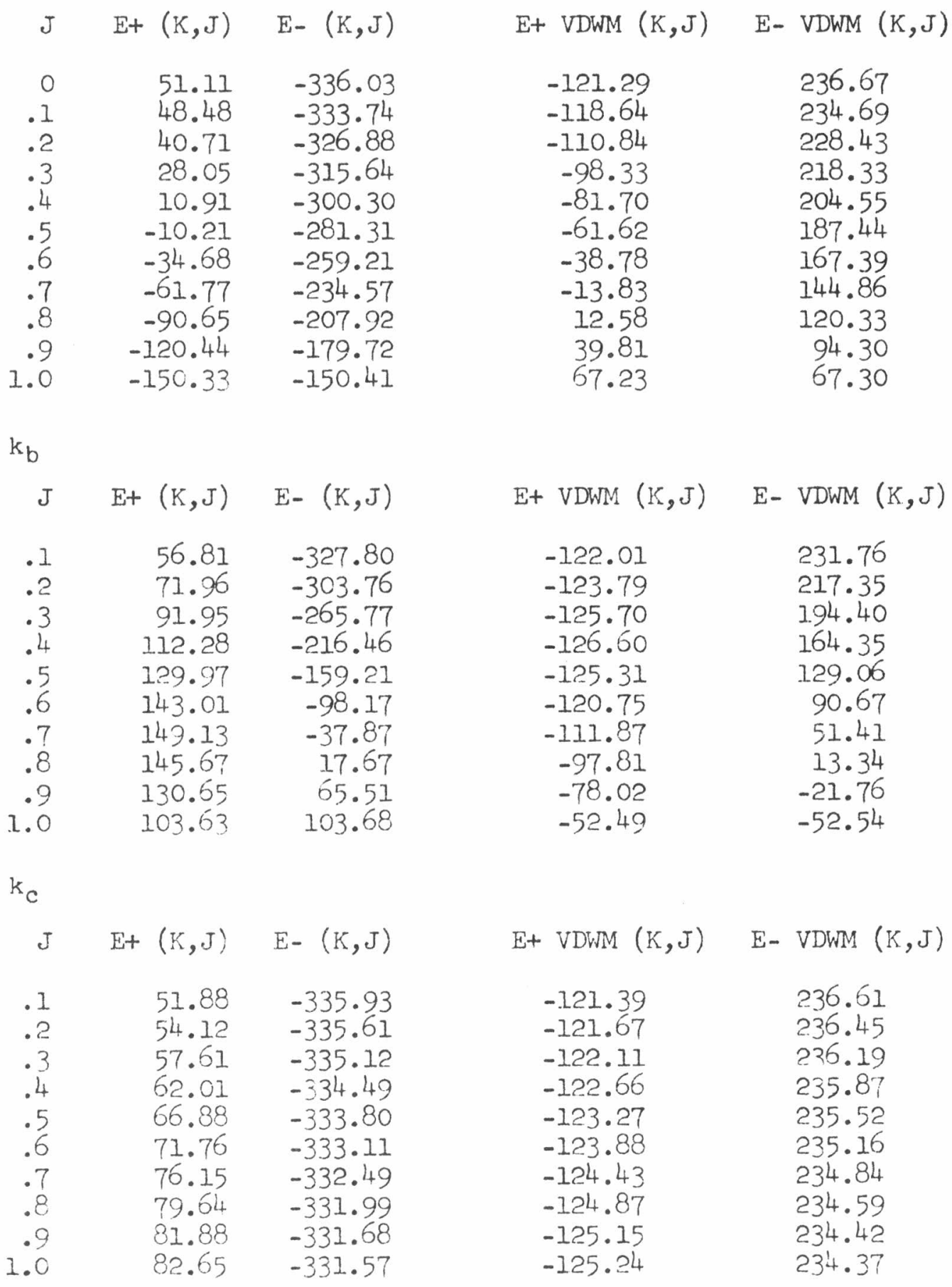


TABLE E-2

ka

$5.0 \mathrm{eV}, \mathrm{A}_{\mathrm{Ig}}$ state

$J \quad E+(K, J) \quad E-(K, J)$

$E+\operatorname{VDWM}(K, J) \quad E-\operatorname{VDWM}(K, J)$

$.1 \quad-1.14$

6.05

5.93

5.73

5.47

5.15

4.78

4.36

3.92

1.82

3.44

2.39

2.93

$$
\begin{aligned}
& -0.58 \\
& -0.59 \\
& -0.63 \\
& -0.68 \\
& -0.76 \\
& -0.87 \\
& -0.99 \\
& -1.13 \\
& -1.26 \\
& -1.41
\end{aligned}
$$

$-2.34$

$-2.30$

$-2.23$

$-2.15$

$-2.05$

$-1.93$

$-1.81$

$-1.68$

$-1.54$

$-1.41$

$\mathrm{k}_{\mathrm{b}}$

$J \quad E+(K, J) \quad E-(K, J)$

$E+\operatorname{VDWM}(K, J) \quad E-\operatorname{VDWM}(K, J)$

$.1 \quad-1.31$

5.93

5.48

4.77

3.85

2.78

1.64

0.51

$-0.54$

$-1.44$

$-2.17$

-0.50
-0.31
-0.03
0.30
0.63
0.93
1.17
1.30
1.32
1.21

$-2.28$

$-2.06$

$-1.73$

$-1.29$

$-0.80$

$-0.29$

0.20

0.64

0.98

-2.98
-2.68

1.21

$\mathrm{k}_{\mathrm{c}}$

$J \quad E+(K, J) \quad E-(K, J)$

E+ VDWM $(K, J)$

E- VDWM $(K, J)$

$.1 \quad-1.21$

6.09

6.09

6.09

6.10

6.11

6.11

6.12

6.13

6.13

$-0.56$

$-2.35$

$-0.54$

$-2.35$

$-0.50$

$-2.36$

$-0.45$

$-2.36$

$-1.36$

$-0.40$

$-0.34$

$-2.36$

$-1.45$

$-0.30$

$-2.36$

$-1.49$

$-0.26$

$-2.36$

$-0.23$

$-2.36$

6.13

$-0.23$

$-2.36$

$-2.36$

$-1.52$ 


\section{REFERENCES}

1. M. Goeppert-Mayer, Ann. Physik 2, 273 (1931).

2. W. Kaiser \& C. G. Garrett, Phys. Rev. Lett. I, 229 (1961).

3. I. D. Abella, Phys. Rev. Lett. 9, 453 (1962).

4. W. L. Peticolas, J. P. Goldsborough \& K. E. Rieckhoff, Phys. Rev. Lett. 10, 43 (1963).

5. J. A. Giordmaine \& J. H. Howe, Phys. Rev. Lett. 11, 207 (1963).

6. R. Braunstein \& N. Ockman, Phys. Rev. 134, A499 (1964).

7. J. J. Hopfield, J. M. Worlock \& K. Park, Phys. Rev. Lett. 11, 414 (1963).

J. J. Hopfield \& J. M. Worlock, Phys. Rev. 137, Al455 (1965).

8. D. A. Kleinman, Phys. Rev. 125, 87 (1962).

9. R. Braunstein, Phys. Rev. 125, 475 (1962).

10. R. Loudon, Proc. Phys. Soc. 80, 952 (1962).

11. J. D. Axe, Phys. Rev. 136, A42 (1964).

12. H. B. Bebb \& A. Gold, Phys. Rev. 143, I (1966).

13. E. Evleth \& W. L. Peticolas, J. Chem. Phys. 41, 1400 (1964).

14. A. Gold \& J. P. Hernandez, Phys. Rev. 139, A2002 (1965).

15. P. A. Franken \& J. F. Ward, Rev. Mod. Phys. 35, 23 (1963).

J. F. Ward, Rev. Mod. Phys. 37, I (19,65).

16. W. L. Peticolas \& K. E. Rieckhoff, J. Chem. Phys. 39, 1347 (1963).

17. W. L. Peticolas, R. Norris \& K. E. Rieckhoff, J. Chem. Phys. 42, $4164(1.965)$.

18. S. Singh \& B. P. Stoicheff, J. Chem. Phys. 38, 2032 (1963).

1.9. J. I. Hall, D. A. Jennings \& K. M. McClintock, Phys. Rev. Lett. 11, $364(1963)$. 
20. S. Singh, W. L. Jones, W. Siebrand, B. P. Stoicheff \& W. G. Schneider, J. Chem. Phys. 42, 330 (1965).

21. M. Iannuzzi \& E. Polacco, Phys. Rev. Iett. 13, 371 (1964).

22. M. Iannuzzi \& E. Polacco, Phys. Rev. 138, A806 (1965).

23. R. G. Kepler, J. C. Caris, P. Avakian \& E. Abramson, Phys. Rev. Lett. 10, 400 (1963).

24. J. M. Worlock in Physics of Quantum Electronics, P. L. Kelley, B. Lax and P. E. Tannenwald eds., McGraw-Hill Book Co., p. 13 (1966).

25. R. Guccione \& J. Van Kranendonk, Phys. Rev. Lett. 14, 583 (1965).

26. R. Pariser \& R. G. Parr, J. Chem. Phys. 21, 466 (1953).

27. R. Pariser, J. Chem. Phys. 24, 250 (1956).

Dr. Pariser kindly provided the author with his unpublished wave functions for $\mathrm{C}_{14} \mathrm{H}_{10}$.

28. E. A. Power \& S. Zienau, Phil. Trans. Roy. Soc. (London) A25l, 427 (1959); Nuovo Cim. 6, 7 (1957).

29. P. I. Richards, Phys. Rev. 13, 254 (1948).

30. P. A. M. Dirac, The Frinciples of Quantum Mechanics (Clarendon Press, Oxford, 1958), 4th ed. p. 248.

31. D. L. Dexter, Solid State Physics, F. Seitz and D. Turnbull eds. vol. 6, p. 353 (Academic Press Inc., New York, 1958).

32. I. D. Landau \& E. M. Lifshiftz, Quantum Mechanics (Non-relativistic Theory) (Addison-Wesley Publishing Co. Inc., 1958) p. $3 \overline{38 .}$

33. M. Inoue \& Y. Toyozawa, J. Phys. Soc. Japan, 20, 363 (1965).

34. See, for example, the review by H. Mangenau, Rev. Mod. Phys. 11,1 (1939).

35. R. Yaris, J. Chem. Phys. 39, 2474 (1963).

36. See for example, A. L. Sklar, J. Chem. Phys. 5, 669 (1937) and M. Goeppert-Mayer \& A. L. Sklar, J. Chem. Phys. 6, 645 (1938).

37. C. A. Coulson, Proc. Phys. Soc. (London) A60, 257 (1948).

38. R. McWeeny, Proc. Phys. Soc. (London) A65, 839 (1952).

39. V. C. Sinclair, J. M. Robertson and A. M. Mathieson, Acta Cryst. 3, 251 (1950). 
40. E. P. Krainov, Optics and Spectroscopy 16, 532 (1964).

41. A. D. Liehr \& C. J. Ballhausen, Phys. Rev. 106, 1161 (1957).

42. J. M. Conway, D. A. Greenwood, J. A. Krumhansl and W. Martienssen, J. Phys. Chem. Solids, 24, 239 (1963).

43. See for example, M. Born andkHuang, The Dynamical Theory of Crystal Lattices (Oxford U. Press, oxford, 1956).

44. F. Seitz, Rev. Mod. Phys. 23, 328 (1951).

45. I. Nakada, J. Phys. Soc. Japan 17, 113 (1962).

46. M. E. Wacks and V. H. Dibeler, J. Chem. Phys. 31, 1557 (1959).

47. See for example, R. S. Knox, Theory of Excitons (Academic Press, New York 1963).

48. See for example, A. S. Davydov, Theory of Molecular Excitons (McGraw-Hill Book Co. Inc., New York, 1962).

49. R. Silbey, J. Jortner, S. A. Rice, J. Chem. Phys. 42, 1515 (1965) and references.

50. A. S. Davydov and E. F. Sheka, Phys. Stat. Sol. 11, 877 (1965).

51. Continuing the expansion in L. I. Shiff, Quantum Mechanics (McGraw-Hill Book Co. Inc., New York, 1955) 2nd ed. p. 177.

52. H. Goldstein, Classical Mechanics (Addison-Wesley Publishing Co. Inc., 1959 p. 107ff.

53. M. S. Brodin and S. V. Marisova, Optics and Spectr. 19, 132 (1965).

54. For a more general discussion of this section, see for example, M. Born and E. Wolf, Principles of Optics (MacMillan Book Co., New York, 1959) p. 675ff.

55. D. Frohlich and H. Mahr, Phys. Rev. Lett. 16, 895 (1966).

56. D. H. McMahon, R. A. Soref and A. R. Franklin, Phys. Rev. Lett. 14, 1060 (1965).

57. There are two typographical errors in the section of ref. 27 we use: in equation (II) there are two terms

"-[ij|ii]" one of them should be changed to read

"-[ij|jj]". Then, in equation (12): " $\Sigma$ " should be changed to read " $\sum_{f \neq i}$. $\quad f \neq k^{\prime}, l^{\prime}$

58. W. Zernik, Phys. Rev. 135, A51 (1964); W. Zernik and R. Klopfenstein, J. of Math. Phys. 6, 262 (1965). 